\title{
Determining the Role of Nudt7 in the Regulation of Cellular CoA Levels and Metabolism
}

\author{
Stephanie Anne Shumar \\ West Virginia University, sashumar@gmail.com
}

Follow this and additional works at: https://researchrepository.wvu.edu/etd

Part of the Biochemistry Commons

\section{Recommended Citation}

Shumar, Stephanie Anne, "Determining the Role of Nudt7 in the Regulation of Cellular CoA Levels and Metabolism" (2019). Graduate Theses, Dissertations, and Problem Reports. 3820.

https://researchrepository.wvu.edu/etd/3820

This Dissertation is protected by copyright and/or related rights. It has been brought to you by the The Research Repository @ WVU with permission from the rights-holder(s). You are free to use this Dissertation in any way that is permitted by the copyright and related rights legislation that applies to your use. For other uses you must obtain permission from the rights-holder(s) directly, unless additional rights are indicated by a Creative Commons license in the record and/ or on the work itself. This Dissertation has been accepted for inclusion in WVU Graduate Theses, Dissertations, and Problem Reports collection by an authorized administrator of The Research Repository @ WVU.

For more information, please contact researchrepository@mail.wvu.edu. 


\title{
Determining the Role of Nudt7 in the Regulation of Cellular CoA Levels and Metabolism
}

\section{Stephanie Anne Shumar}

\author{
Dissertation submitted \\ to the School of Medicine \\ at West Virginia University \\ in partial fulfillment of the requirements for the degree of \\ Doctor of Philosophy in \\ Biochemistry and Molecular Biology \\ Roberta Leonardi, Ph.D., Chair \\ Frank B. Hillgartner, Ph.D. \\ Lisa Salati, Ph.D. \\ David Smith, Ph.D. \\ Werner Geldenhuys, Ph.D \\ Morgantown, West Virginia \\ 2019
}

Keywords: Coenzyme A, Nudix hydrolase, liver metabolism, fatty acid oxidation, bile acid synthesis 


\begin{abstract}
Coenzyme A (CoA) is an essential cofactor required for hundreds of metabolic processes. Because it is such a critical cofactor, CoA levels are tightly regulated. In the fasted state and in diabetic mice, the concentration of CoA increases dramatically in the liver. This phenotype is associated with constitutively low CoA degradation, a process that is emerging as a potentially important mechanism for CoA regulation. Nudt7 and Nudt19 are two mammalian peroxisomal enzymes with CoA-degrading activity, which are highly expressed in the liver and kidney, respectively. Limited information is available on the biochemistry of Nudt7 and Nudt19; the structural basis for their distinct features and the extent to which Nudt7 contributes to maintaining homeostatic CoA levels in vivo are currently unknown. We used a combination of techniques including mutagenesis, molecular modeling, and enzymatic assays on purified proteins, plus metabolomics and measurement of fatty acid oxidation in whole tissue homogenates and intact hepatocytes to: 1) characterize the biochemical, structural, and regulatory properties of Nudt7 and Nudt19 and 2) determine the effects that manipulations of Nudt7 expression have on CoA levels and lipid metabolism in mouse liver. This research establishes the importance of Nudt7dependent CoA degradation in the regulation of select acyl-CoA species and the output of peroxisomal metabolic pathways such as bile acid synthesis and peroxisomal fatty acid oxidation.
\end{abstract}




\section{Acknowledgments}

I would like to thank the many colleagues and mentors that have supported me on this amazing and challenging journey. First, I would like to thank my advisor, Dr. Roberta Leonardi, for accepting me as her "first-born" into her lab right after starting her lab at WVU, providing me with the tools to be an outstanding scientist, and supporting me in my atypical career goals. I would like to thank my committee members, Dr. Lisa Salati, Dr. Brad Hillgartner, Dr. Werner Geldenhuyes, and Dr. David Smith for their valuable ideas, input, and constructive criticism. I would also like to thank the members of the Monday "RNA and Metabolism" group meeting and the Biochemistry department for their valuable input and

feedback. Finally, thank you to Evan and Debbie for being great coworkers and friends for the past 5 and $1 / 2$ years.

Outside of the department, I have had the support of a multitude of people that I cannot thank enough. First and foremost, I would like to thank my parents for always supporting me $1000 \%$ in everything that I do and thinking that I am the best child ever (even if that conclusion is biased). I would like to thank my best friend Norma for being the closest thing that I will ever have to a sister and supporting me through some of the hardest moments of the last several years. I would also like to thank Dudley and Kathleen for being great friends and willing to drop anything and come to my aid whenever I needed ideas, assistance, to vent about science, or just someone to sit and vegetate on the couch with. To everyone else that has supported me in science and in my life, thank you for being there for me. 


\section{Abbreviations}

\begin{tabular}{|c|c|}
\hline AAV & Adeno-associated virus \\
\hline ACOT & Acyl-CoA thioesterase \\
\hline ACOX & Acyl-Coenzyme oxidase \\
\hline ALT & Alanine aminotransferase \\
\hline AST & Aspartate aminotransferase \\
\hline AT-1 & Acetyl-CoA transporter 1 \\
\hline Baat & Bile Acid-CoA: amino acid N-acyltransferase \\
\hline CBP & CREB-binding protein \\
\hline CDCA & Chenodeoxycholic acid \\
\hline CoA & Coenzyme A \\
\hline Coasy & CoA synthase \\
\hline Crot & Carnitine octanolytransferase \\
\hline Cyp7a1 & Cholesterol $7 \alpha$-hydroxylase \\
\hline ER & Endoplasmic reticulum \\
\hline GAPDH & Glyceraldehyde-3-phosphate dehydrogenase \\
\hline GusB & $\beta$-Glucuronidase \\
\hline HNF-4a & Hepatocyte nuclear factor- $4 \alpha$ \\
\hline ITS & Isothermal titration calorimetry \\
\hline LCA & Long-chain acyl \\
\hline M199 & Medium 199 \\
\hline $\mathbf{m B B}$ & Monobromobimane \\
\hline MCA & Medium-chain acyl \\
\hline Nr0b2, SHP & Small heterodimer partner \\
\hline PanK & Pantothenate kinase \\
\hline PKAN & Pantothenate Kinase-Associated Neurodegeneration \\
\hline PMP70 & ATP-binding cassette, sub-family D, member 3 \\
\hline PPARa & Peroxisome proliferator-activated receptor $\alpha$ \\
\hline PTS & Peroxisomal targeting sequence \\
\hline SCPx & Sterol carrier protein $\mathrm{X}$ \\
\hline TPX2 & Targeting protein for Xklp2 \\
\hline VLCA & Very long-chain acyl \\
\hline
\end{tabular}




\section{Table of Contents}

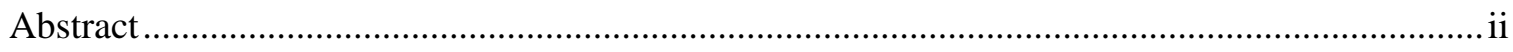

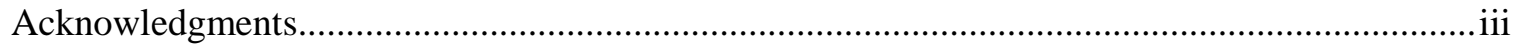

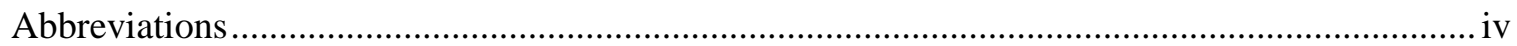

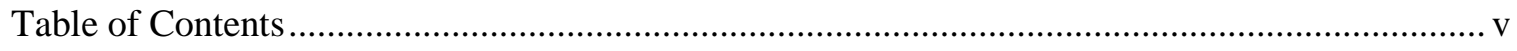

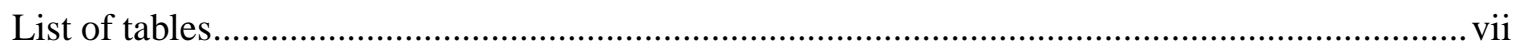

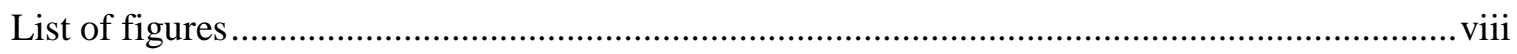

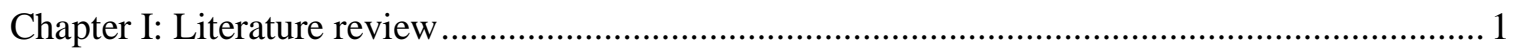

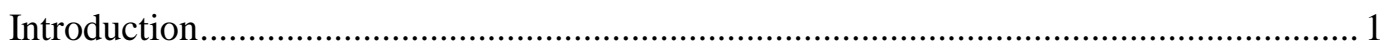

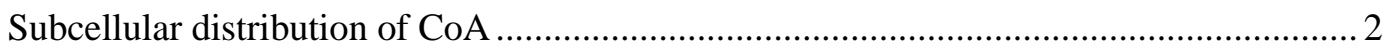

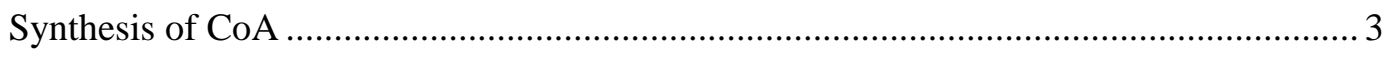

Regulation of CoA synthesis ................................................................... 4

Problems associated with impaired CoA synthesis............................................ 5

Problems associated with supraphysiological CoA levels ................................ 6

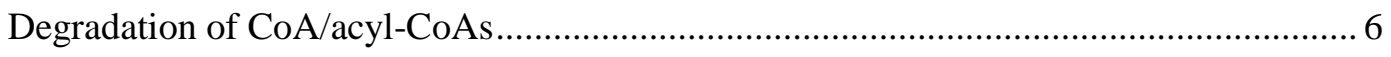

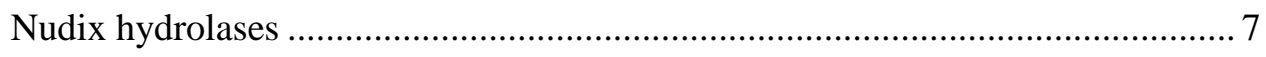

Mammalian CoA-degrading Nudix hydrolases .............................................. 8

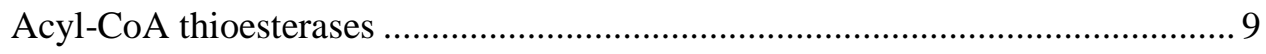

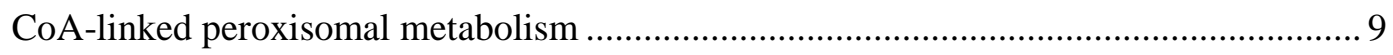

Peroxisomal bile acid synthesis .................................................................. 10

Peroxisomal $\beta$-oxidation and the connection to the mitochondria....................... 10

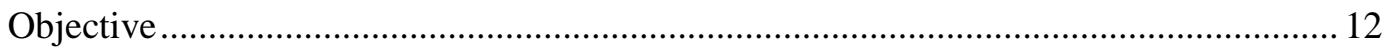

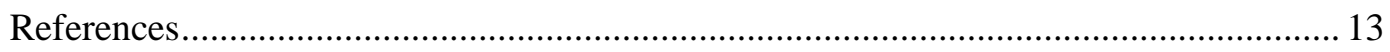

Chapter II: Characterization of the biochemical and structural properties of Nudt7 and Nudt19 . 17

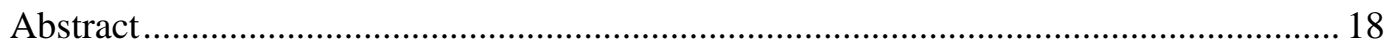




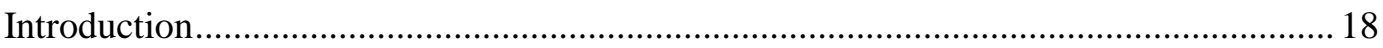

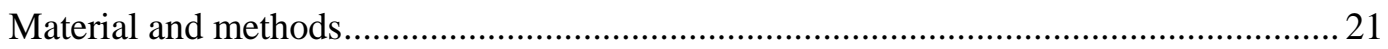

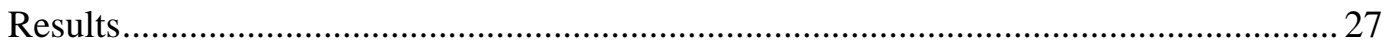

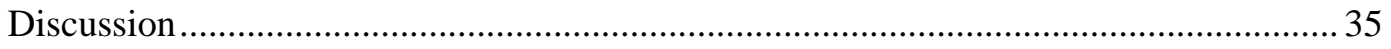

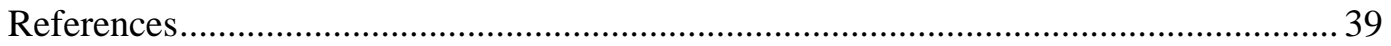

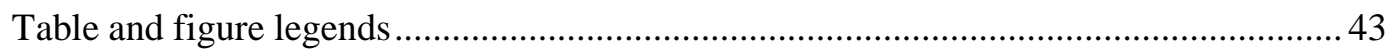

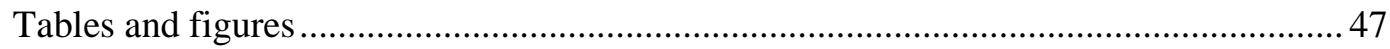

Chapter III: Identification of the in vivo CoA species and subcellular CoA pool(s) regulated by Nudt7 56

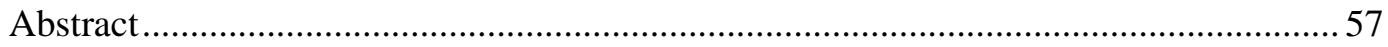

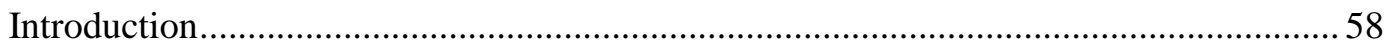

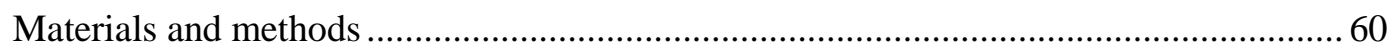

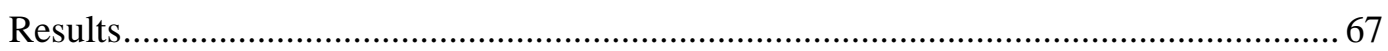

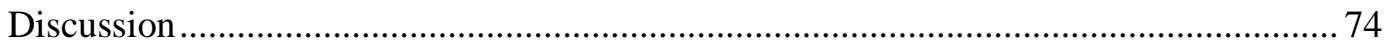

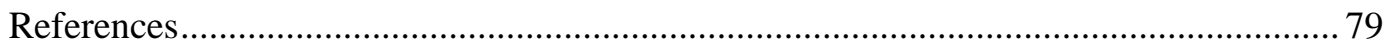

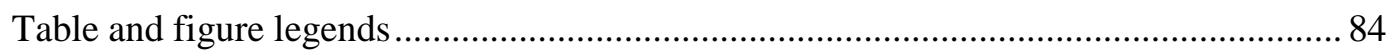

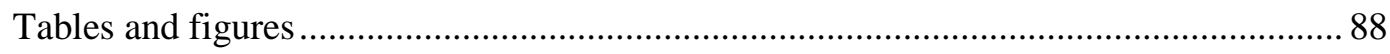

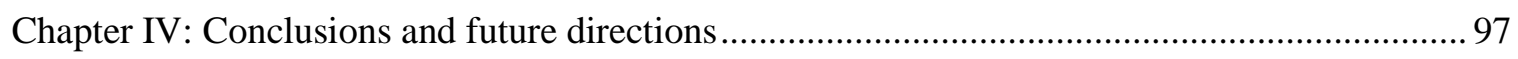

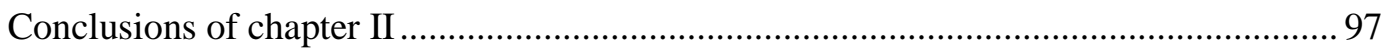

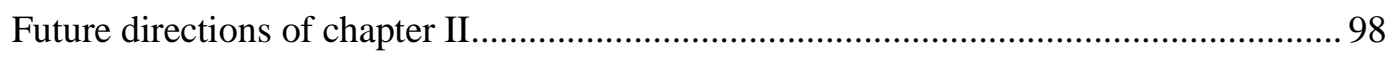

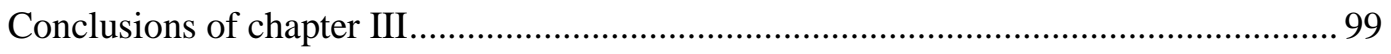

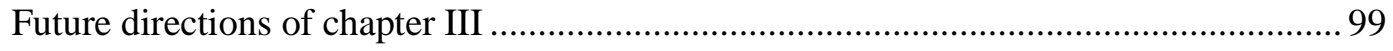

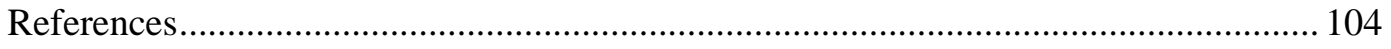




\section{List of tables}

Table 2.1. Effect of selected metabolites on the activity of Nudt19 and Nudt7....................... 47

Table 2.2. Kinetic parameters of Nudt19 and Nudt7 mutants and chimeras. ............................. 48

Table 3.1. Selected features of fed and $24 \mathrm{~h}$ fasted GFP and Nudt7 mice............................... 88

Table 3.2. Over-representation enrichment analysis of the genes differentially expressed

between Nudt7 and GFP mice in the fasted state. ............................................................... 89

Supplementary Table 3.S1. Global metabolic profiling ..................................................... 90

Supplementary Table 3.S2. Acyl-CoA LC/MS/MS parameters .............................................. 90

Supplementary Table 3.S3. Nudt7-AAV RNAseq results.................................................... 90 


\section{List of figures}

Figure 1.1. The regulation of CoA through synthesis and degradation.

Figure 2.1. In vitro activity and tissue distribution of Nudt19 and Nudt7 .............................. 49

Figure 2.2. Effect of Nudt19 deletion on kidney CoA levels. ............................................... 50

Figure 2.3. Localization of Nudt19 to the peroxisomes...................................................... 51

Figure 2.4. Nudt19 expression in the kidneys of fed and fasted mice...................................... 52

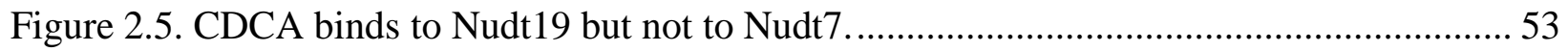

Figure 2.6. Effect of exchanging the CoA and Nudix boxes of Nudt19 and Nudt7.................. 54

Figure 2.7. Homology model of Nudt19 with magnesium ion and free CoA bound. ................. 55

Figure 3.1. Changes in hepatic CoA content, Nudt7 mRNA, and activity with fasting.............. 91

Figure 3.2. Liver-specific overexpression of Nudt7 and GFP and effect on hepatic CoA levels. 92

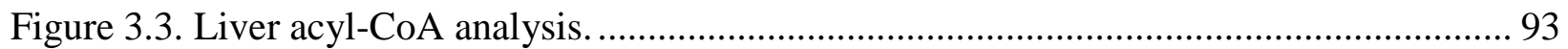

Figure 3.4. Global metabolic profiling of $24 \mathrm{~h}$ fasted Nudt7 and GFP livers............................ 94

Figure 3.5. Nudt7 overexpression in the fasted state decreases the hepatic bile acid content...... 95

Figure 3.6. Nudt7 overexpression in the fasted state decreases the rate of peroxisomal fatty acid

oxidation. 96 


\section{Chapter I: Literature review}

\section{$\underline{\text { Introduction }}$}

Coenzyme A (CoA) is an essential and ubiquitous cofactor that plays a key role in energy metabolism. It is estimated that about $4 \%$ of all cellular enzymes utilize CoA as a cofactor and that it may be involved in at least 100 metabolic reactions (1,2). CoA was identified from hog liver in 1947 and its structure later determined in $1953(3,4)$. The structure of CoA consists of a 3',5'-ADP, phosphopantothenate, and cysteamine. The reactive thiol group of CoA reacts with carboxylic acids to form thioesters, thus CoA acts as an acyl carrier to move various fatty acids across membranes and during intermediate steps of numerous metabolic pathways (2). The CoA pool also includes acetyl-CoA, the acetylgroup donor for protein acetylation, an essential post-translational modification that regulates the function of histones and thousands of other proteins. Formation of short, medium, and long chain acyl-CoA thioesters is essential for metabolic pathways found in the cytosolic, mitochondrial, and peroxisomal compartments of the cell (5). Because of its ability to regulate the output of several metabolic pathways, the concentration of CoA in various tissues is tightly regulated and adjusted depending on the metabolic state. In particular, CoA levels increase in the transition from the fed to the fasted state to support the switch from glucose to fatty acid oxidation in metabolically flexible organs like the liver and kidneys. Importance of such a tight regulation of CoA concentration is underscored by the fact that abnormally high and dysregulated CoA levels in the liver actively contribute to the hyperglycemia that characterizes mouse models of type 2 diabetes, while increased malonyl-CoA in the muscle of obese subjects is associated with increased lipogenesis $(6,7)$. Conversely, inhibition of CoA synthesis by genetic manipulations or treatment with the chemical inhibitor Hopan, causes changes in global protein acetylation and hypoglycemia (8-10). Combined, this evidence underscores the importance of regulating CoA levels. 


\section{$\underline{\text { Subcellular distribution of CoA }}$}

Mitochondria, peroxisomes, and the cytosol/nucleus contain separate pools of CoA that are important for the processes that occur in each of these cellular compartments. The highest concentration of CoA is found in the mitochondria, estimated at 4 to $5 \mathrm{mM}(11,12)$. CoA is important in the mitochondria for several metabolic pathways such as ketogenesis, and the oxidation of pyruvate and fatty acids. The second subcellular CoA pool (approximately $0.1 \mathrm{mM}$ ) is the cytosolic pool which is important for complex lipid synthesis that occurs there (12). The cytosolic CoA pool can freely equilibrate with the nuclear pool since all CoA species are small enough to cross the nuclear pores. Within the nucleus, acyl-CoAs contribute to histone acetylation and regulation of gene expression for pathways such as spermatogenesis and ketogenesis (13). For example, long-chain acyl (LCA)-CoAs are potent down regulators of the nuclear thyroid hormone receptor $(14,15)$ and various acyl-CoAs are able to regulate transcriptional activity of hepatocyte nuclear factor $4 \alpha(\mathrm{HNF}-4 \alpha)(16)$. The final and smallest pool of CoA (approximately 0.3 to 0.7 $\mathrm{mM}$ ) is found in the peroxisomes. The pool of CoA in this compartment is used for the oxidation of lipids that cannot be processed in the mitochondria, bile acid synthesis, and for the synthesis of ether lipids. $(12,17)$. It is currently unknown how the individual pools of CoA are regulated.

The endoplasmic reticulum (ER) also contains its own pool of acetyl-CoA. Acetyl-CoA is transported into the ER through acetyl-CoA transporter 1 (AT-1) (18). Once inside the ER, acetyl-CoA lysine acetyltransferases use the acetyl-CoA to acetylate lysine residues on a long list of proteins involved in autophagy and protein quality control. Reduced acetyl-CoA import into the ER leads to neurodegenerative disorders, disruptions of the immune system, and development of various cancers (19). 


\section{Synthesis of CoA}

Regulation of the biosynthetic

pathway is a major mechanism to adjust CoA levels in the entire cell. CoA is synthesized from pantothenate (vitamin $\mathrm{B}_{5}$ ), ATP, and cysteine through a series of five reactions (4). First, pantothenate kinase (PanK) phosphorylates pantothenate to form 4'phosphopantothenate (Fig. 1.1). Next, 4'-phosphopantothenoylcysteine (PPCS) synthase catalyzes the condensation of 4'phosphopantothenate with cysteine to form 4'-phosphopantothenoylcysteine. Then 4'-phosphopantothenoylcysteine decarboxylase (PPCDC)

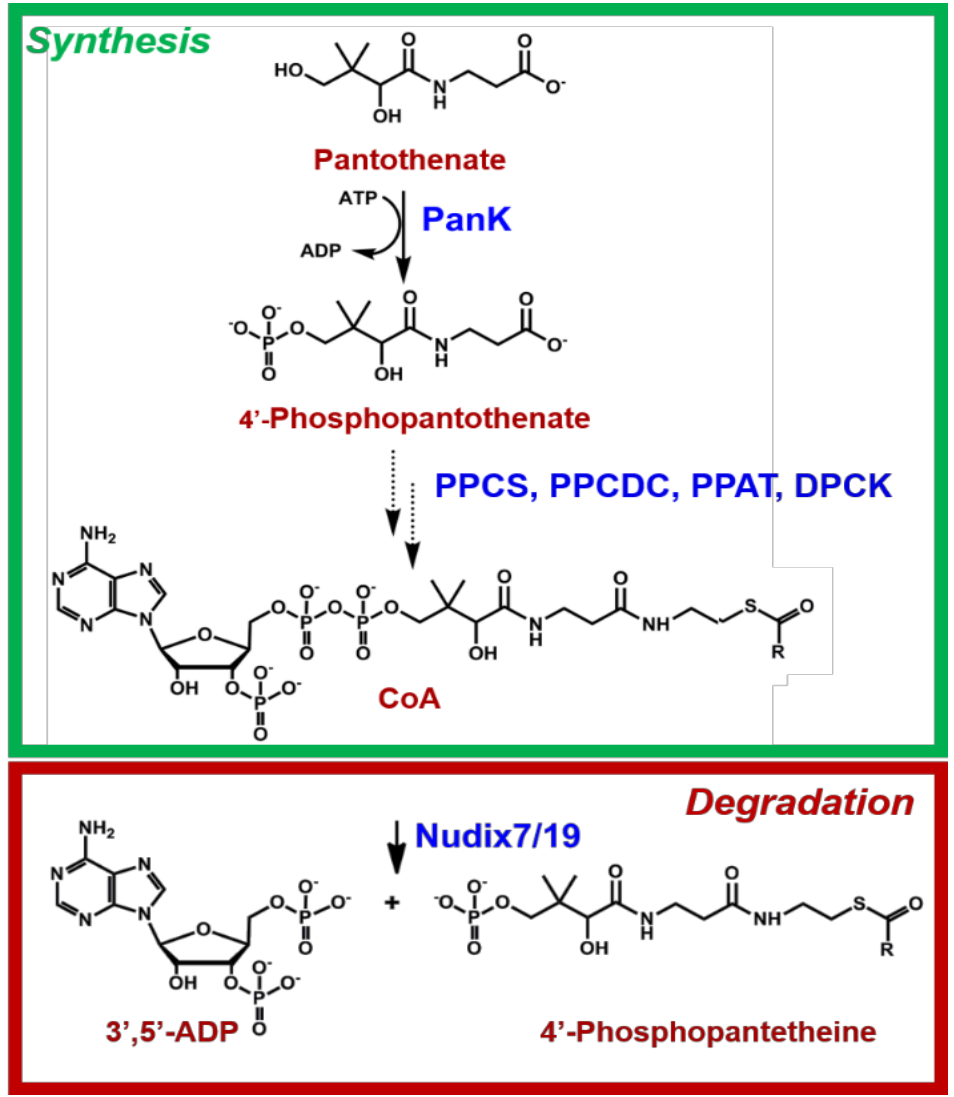

Figure 1.1. The regulation of CoA through synthesis and degradation. Pantothenate is converted into 4'Phosphopantothenate by the PanKs in the CoA synthesis pathway. CoA is degraded into 3',5'-ADP and 4'Phosphopanteteine by Nudt7 and Nudt19.

decarboxylates this intermediate forming 4'-phosphopantetheine. In the fourth step of the biosynthetic pathway, phosphopantetheine adenylyltransferase (PPAT) converts 4'-phosphopantetheine into dephospho-CoA. Finally, dephospho-CoA kinase (DPCK) phosphorylates dephospho-CoA at the 3'-OH of the ribose sugar producing CoA (2). In eukaryotes, the last two steps are catalyzed by a bifunctional enzyme called CoA synthase (Coasy). The subcellular localization of Coasy is still a matter of debate. This enzyme has been localized to both the mitochondrial outer membrane with the active site exposed to the cytosol and to the mitochondrial matrix. In the first case, CoA would be synthesized in the cytosol; in the 
second case, CoA would be synthesized in the mitochondrial matrix and require a specific transporter to exit this organelle and reach other subcellular compartments.

\section{Regulation of CoA synthesis}

CoA biosynthesis is controlled by PanK, the enzyme that catalyzes the first and rate-limiting step in the pathway. Regulation of PanK occurs through feedback inhibition by the cellular levels of free CoA and CoA thioesters (20-22). In mammals, there are three Pank genes that are responsible for the expression of four catalytically active PanK enzymes: PanK1 $\alpha$, PanK1 $\beta$, PanK2, and PanK3 (23). The four PanK isoforms share $>80 \%$ sequence identity but possess distinct $\mathrm{N}$-termini that direct them to different subcellular compartments. Both of the PanK1 isoforms are expressed from the Pank1 gene through alternative initiation sites (24), and are the least sensitive to feed-back inhibition by CoA species. PanK1 $\alpha$ resides in the nucleus and is highly expressed in kidney and heart. PanK $1 \beta$ is cytosolic and highly expressed in the liver. PanK2 is localized to the mitochondria in humans and found in the cytosol in mice $(25,26)$. In humans, this isoform is highly expressed in the brain. PanK3, another cytosolic isoform, is ubiquitously distributed and, together with PanK2, is the most stringently regulated PanK isoform $(23,27)$. Both the human PANK2 and mouse PanK2 are potently inhibited by acetyl-CoA and the inhibition is released by palmitoylcarnitine (26). Competition between acetyl-CoA and palmitoylcarnitine for the active site allows PanK2 to act as a "sensor" of the status of mitochondrial fatty acid oxidation enabling the cell to increase or decrease CoA biosynthesis in response to mitochondrial metabolism (26). PanK3 shares approximately $79 \%$ amino acid identity to PanK1 $\beta$, is localized to the cytosol, and most abundant in the liver and kidneys. Whereas PanK1 $\alpha$ and PanK1 $\beta$ are more refractory to inhibition by CoA species, PanK3 is inhibited by to free CoA, acetyl-CoA, malonyl-CoA, and palmitoyl-CoA. Though PanK3 is expressed at the highest levels in the liver compared to other tissues, it only accounts for about $30 \%$ of the total PanK protein in the liver. PanK $1 \alpha / \beta$ accounts for $70 \%$ of the liver's total PanK protein pool.

In addition to negative feedback by CoA on the PanKs, various hormones and metabolites can modulate the rate of this step of the CoA biosynthetic pathway. Glucose (28), palmitate, pyruvate, $\beta$ - 
hydroxybutyrate and insulin $(29,30)$ inhibit CoA biosynthesis while glucagon and glucocorticoids stimulate CoA synthesis (31). These early studies utilized flux analysis to measure the production of CoA through the pathway, therefore the mechanism by which these metabolites regulate CoA synthesis remains to be elucidated.

\section{Problems associated with impaired CoA synthesis}

Disruption of CoA biosynthesis leads to severe metabolic consequences. Pank1 $1^{-/}$mice exhibit a $40 \%$ decrease in the concentration of CoA levels in the liver and the inability to sustain the increase in the rate of fatty acid $\beta$-oxidation that characterizes the fed-to-fasted transition in this organ (32). This, in turn, blunts the ability of the liver to synthesize glucose de novo (gluconeogenesis) during a fast, a process that is activated by the products (NADH and acetyl-CoA) of fatty acid oxidation. As a consequence, Pank $1^{-/-}$ mice exhibit fasting hypoglycemia (32). This phenotype is milder compared to mice treated with HoPan, a pantothenate analog that inhibits all the PanK isoforms. HoPan-treated mice exhibit an $80 \%$ reduction in liver and kidney CoA levels and rapidly die of hypoglycemia, even if food is available (10). Mutations of

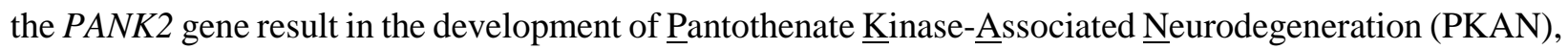
a rare autosomal recessive disorder characterized by dystonia and accumulation of iron in the basal ganglia generally occurring before 10 years of age (33). Pank2 $2^{-/-}$mice have been generated, but they do not show any sign of neurodegeneration. Instead, these mice exhibit male sterility and retinal degeneration (34). Generation of Pank1/Pank2 ${ }^{-/}$mice exhibit a more severe decrease in the concentration of CoA in both the liver and brain indicating that both isoforms can compensate for each other. The double knockout mice also exhibit severe hypoglycemia, liver triglyceride accumulation, and hyperketonemia leading to death by 3 weeks of age (35).

Inhibition of the final steps of CoA synthesis also cause abnormalities. A recent study of Coasy in cells have found that knockdown of Coasy leads of hyperacetylation of mitotic proteins result in mitotic defects and multi-nucleation (36). While CoA levels were significant decreased upon Coasy knockdown, an additional function of the enzyme was identified from this study; during mitosis Coasy interacts with the 
histone acetyltransferase, CREB-binding protein (CBP), and inhibits its activity. Loss of Coasy resulted in persistent CBP acetylation of its downstream target, Targeting Protein for Xklp2 (TPX2), resulting in prolonged TPX2 stabilization, activation of aurora kinase, and mitotic defects. Generation and analysis of Coasy in a mouse model has yet to be completed.

\section{Problems associated with supraphysiological CoA levels}

The link between CoA levels and gluconeogenesis was confirmed in a recent study on leptindeficient $\left(\mathrm{Lep}^{-/}\right)$mice, a well-established model of obesity-induced type 2 diabetes (37). Lep $p^{-/-}$mice are obese and contain abnormally high hepatic CoA levels that no longer respond to nutritional regulation. Deletion of Pank1 in $\mathrm{Lep}^{-/-}$mice decreased liver CoA levels and significantly improved hyperglycemia and hyperinsulinemia, independent of changes in body weight. Overexpression of human PANK2 in a transgenic mouse model led to elevated skeletal muscle CoA levels resulting in reduced skeletal muscle mass, reduced ATP levels, increased oxidative stress, impaired exercise tolerance, and reduced grip strength (38). Interestingly, vitamin B12 deficiency has also been found to cause a significant increase in total liver CoA levels and a large accumulation of propionyl-CoA and methylmalonyl-CoA; however, no downstream metabolic consequences of this increase were found (39).

\section{Degradation of CoA/acyl-CoAs}

Degradation is another possible way to rapidly modulate the concentration of cellular CoA. In the liver and kidneys, degradation of CoA is necessary in the transition from the fasted to the fed state to decrease the levels of the cofactor that accumulated due to a starvation stimulated increase in synthesis. The existence of a pathway for CoA degradation is also supported by the fact that mice treated with HoPan (the chemical inhibitor of the PanK enzymes) exhibit a rapid decrease in liver and kidney CoA levels, indicating the presence of an enzyme(s) that could break down (acyl-)CoA. Early studies in rat liver identified three possible enzymes capable of degrading CoA. Fractionation and kinetic analysis provided evidence that one such enzyme was localized to the lysosomes, one was in the nuclear fraction, and one 
was found in the microsomal fraction (40). The lysosomal enzyme was found to dephosphorylate CoA while the microsomal and nuclear enzymes were both thought to be pyrophosphatases capable of hydrolyzing CoA and dephospho-CoA (41). The degradation of CoA by these enzymes was thought to be a sequence of four steps starting with the removal of the 3'-phosphate to form dephospho-CoA, followed

by degradation to 4'-phosphopantetheine, then dephosphorylation to form pantetheine, and finally degradation of pantetheine into pantothenic acid and cysteamine (42).

\section{Nudix hydrolases}

Nudix hydrolases are a superfamily of diphosphohydrolases found in most eukaryotes, prokaryotes, archaea, and viruses. The members of this family catalyze the hydrolysis of the bond between a nucleoside diphosphate linked to another moiety $\underline{X}$ (Nudix). All members of this family possess the signature Nudix box motif $-\mathrm{Gx}_{5} \mathrm{Ex}_{5}(\mathrm{UA}) \mathrm{xRE}(\mathrm{UA}) \mathrm{xEExGU}$ - that is essential for binding a divalent cation, typically magnesium, required for the hydrolysis of phosphate-phosphate or phosphate-carbon bonds. Members of this family were originally recognized as exhibiting a "housecleaning” function as they degrade metabolites that could be potentially hazardous if they accumulate in the cell (43). The number of Nudix hydrolases in each organism varies from zero to 30 . The substrate specificity of these enzymes is determined by other specific motifs in the sequence. For example, Nudix hydrolases specific for CoA and CoA thioesters contain the amino acid sequence LLTxR[SA] $\mathrm{x}_{3} \mathrm{Rx}_{3} \mathrm{Gx}_{3} \mathrm{FPGG}$ (PROSITE accession number PS01293), near the Nudix box motif (44). CoA-degrading Nudix hydrolases are conserved from bacteria to humans. The first CoA-degrading Nudix hydrolase discovered was Pcd1p, a Saccharomyces cerevisiae Nudix hydrolase. Studies of this enzyme determined that it degrades free CoA and some acyl-CoA species into 3',5'-ADP and 4'-phosphopantetheine (Fig. 1.1). Pcd1p was also determined to be the first peroxisomal Nudix hydrolase as evidenced by the presences of a peroxisomal targeting sequence (PTS) 2 and cellular localization studies (45). Soon after, other CoA-degrading enzymes were discovered in Deinococcus radiodurans, and the peroxisomes of Caenorhabditis elegans, mice, and humans (46-49). Currently, there are two available structures of these CoA-degrading enzymes. The first is that of D. radiodurans. The 
structure of the enzyme with $\mathrm{Mg}^{2+}$ bound has provided some insight into possible key residues for catalysis and has helped define the CoA-binding motif through modeling of the substrate into the putative active site. The Nudix motif, a helix-loop-helix structure, contains three glutamate residues that coordinate the magnesium ion. Within the CoA-binding motif are an arginine and a serine residue that are potentially involved in the coordination of the leaving products (phosphopantetheine or 3',5'-ADP) after hydrolysis (50). The crystal structure of human NUDT7 has recently been solved (PDB ID: 5T3P) (51), however, the crystal structure of the human enzyme does not contain bound $\mathrm{Mg}^{2+}$. Both enzyme structures were solved without a CoA substrate. Thus, the contribution of the CoA motif to CoA binding and the organization of the CoA binding site in these enzymes are currently unknown.

\section{Mammalian CoA-degrading Nudix hydrolases}

There are currently only two confirmed peroxisomal CoA-degrading Nudix hydrolases found in mammals, Nudt7 and Nudt19. Nudt7 is found in highest quantities in the liver, with low but detectable levels in brown adipose tissue and heart. Nudt19 is expressed in highest concentrations in the kidneys (49). Both Nudt7 and Nudt19 have a C-terminal PTS1 sequence which directs them to the peroxisomes $(48,49)$. Nudt7 has a molecular weight of $27 \mathrm{kDa}$, a typical size for a Nudix hydrolase, while Nudt19 is larger at about 42 kDa. Nudt7 and Nudt19 exhibit only a modest sequence similarity. One key difference in the amino acid sequence of Nudt7 and Nudt19 is the presence of an approximate 45 amino acid insertion in the Nudix motif of Nudt19. Though small insertions have been observed in the Nudix box of other Nudix hydrolases, this large insertion sequence is unique to Nudt19. The purpose of the sequence is currently unknown. Analysis of Nudt7 activity has shown that it is a promiscuous enzyme, catalyzing the degradation of free CoA as well as short- and medium-chain acyl-CoA species. In contrast, Nudt19 is much more selective, catalyzing the degradation of free and medium-chain acyl-CoAs but not, for example, acetyl-CoA

$(49,52)$. Nudt7 mRNA and activity change in the liver in response to the metabolic state. The amount of Nudt7 mRNA and activity is high in the fed state and decreases in the fasted state, correlating to the increase 
to hepatic CoA levels. It has remained undetermined to what extent, if any, the change in Nudt7 activity in the peroxisomes contributes to the change in liver CoA levels and/or peroxisomal metabolism.

\section{Acyl-CoA thioesterases}

In addition to complete degradation by the Nudix hydrolases, CoA thioesters can also be hydrolyzed to free CoA and free fatty acids by acyl-CoA thioesterases (ACOT) $(53,54)$ to modulate certain metabolic pathways. There are two families of ACOTs. Type I ACOTs are members of the $\alpha / \beta$-hydrolase protein family and exhibit a conserved "catalytic triad" of a serine, histidine, and aspartic acid in their active sites (55). Type II ACOTs have lower sequence similarity but all contain the conserved "HotDog domain”; a structural motif consisting of a seven-stranded anti-parallel $\beta$-sheet “bun” wrapped around a hydrophobic five-turn $\alpha$-helical sausage, with a third layer of “condiment” loops over the helix (53). ACOTs from both families are localized to the various subcellular compartments. Six different ACOTs (ACOT3, 4, 5, 6, 8, and 12) in mice and two (ACOT4 and 8) in humans localize to the peroxisomes, suggesting that hydrolysis of acyl-CoAs may be a way to quickly modulate lipid metabolism in the peroxisomes independently of CoA biosynthesis. Each of the peroxisomal ACOTs has a defined substrate specificity but combined, they all contribute to the hydrolysis of short-, medium-, and long-chain acyl-CoAs, bile acid-CoAs, and branched-chain acyl-CoAs. Murine Acot3, Acot4, Acot5, and Acot8 are thought to be involved in regulating peroxisomal $\beta$-oxidation (56,57). Acot3 is specific for long-chain acyl-CoAs, Acot5 is specific for medium-chain acyl-CoAs, and Acot4 hydrolyzes only succinyl-CoA and glutaryl-CoA. Human ACOT4 exhibits activity against all of these substrates and is thought to have "replaced" the activity of Acot3 and Acot5 in mice (58). It has been hypothesized that these enzymes hydrolyze acyl-CoAs to allow the free fatty acid to be exported from the peroxisomes and utilized in mitochondrial $\beta$-oxidation and then Nudt7 and Nudt19 hydrolyze the resulting free CoA to prevent a toxic buildup.

\section{CoA-linked peroxisomal metabolism}

While peroxisomes have been historically associated with the containment and breakdown of hydrogen peroxide that is formed from the oxidation of other substrates, there are several other metabolic 
processes that occur within these organelles. These processes include $\beta$-oxidation of very long-chain fatty acids, branched-chain fatty acids, bile acid intermediates, and long-chain dicarboxylic acids, $\alpha$-oxidation of fatty acids, glyoxylate detoxification, ether phospholipid biosynthesis, and bile acid synthesis. Many of these processes require free CoA for various steps in the pathways and result in the formation of short chain acyl-CoA species. For example, the thiolase enzymes require a molecule of free CoA for each round of $\beta$ oxidation to bind to the shortened fatty acid from which acetyl-CoA has been released. Similarly, sterol carrier protein X (SCPx) requires free CoA for the last step of bile acid formation, which releases propionylCoA $(59,60)$. Above all else, peroxisomal acyl-CoA synthetases require free CoA to activate fatty acids and steroid derivatives when they enter the peroxisomes so that they can be further metabolized (61).

\section{Peroxisomal bile acid synthesis}

Peroxisomes play a critical role in the synthesis of bile acids, as illustrated by the bile acid abnormalities observed in individuals with peroxisomal biogenesis disorders $(62,63)$. The two primary bile acids chenodeoxycholic acid (CDCA) and cholic acid are formed from cholesterol. Cholesterol is modified on the sterol side chain through several steps and activated to a CoA thioester in the ER. Once conjugated to CoA, the C27 bile acid intermediate can enter the peroxisome to complete its biosynthesis and be conjugated to taurine or glycine (59). Once the mature bile acid is conjugated to taurine or glycine, it is excreted into the bile. The last step in the biosynthesic process, catalyzed by SCPx, requires an additional molecule of free CoA and results in the formation of proponyl-CoA. This step has been previously identified as a key step in completion of bile acid synthesis, as loss of SCPx results in an accumulation of bile acid intermediates $(64,65)$. Interestingly, it is known that onset of obesity and Type 2 diabetes, which are associated with elevated, unregulated CoA levels, leads to an elevation in the total bile acid pool even upon administration of bile acid sequestrants (66). However, there has never been any direct connection established between peroxisomal CoA levels and the rate of bile acid synthesis.

\section{Peroxisomal $\beta$-oxidation and the connection to the mitochondria}


Both mitochondria and peroxisomes contribute to fatty acid oxidation; however, each organelle contains distinct sets of enzymes. $\beta$-Oxidation of the majority of short-, medium-, and long-chain fatty acids is localized primarily to the mitochondria and supports the production of ATP through oxidative phosphorylation (67). $\beta$-Oxidation of very long-chain fatty acid occurs almost exclusively in the peroxisomes since mitochondria lack a very long-chain acyl-CoA synthetase to activate these fatty acids to the correspondent acyl-CoAs. Once activated, the very long-chain acyl (VLCA)-CoAs are then shortened to medium-chain acyl (MCA)-CoAs through the peroxisomal $\beta$-oxidation pathway. Shortening of VCLACoAs in the peroxisomes has been well established as a crucial and irreplaceable step. Disruption of the gene coding for fatty acyl-coenzyme A oxidase (ACOX), the first enzyme in the peroxisomal $\beta$-oxidation pathway, results in the development of peroxisomal metabolic disorders (68-71). These disorders are characterized by a buildup of very long chain fatty acids in the blood, growth retardation, sterility, and neurological abnormalities.

Once the VLCA-CoAs are shortened to MCA-CoAs, carnitine octanoyltransferase (Crot) then converts the MCA-CoAs into MCA-carnitines, which can cross the peroxisomal and mitochondrial membrane and feed into the mitochondrial fatty acid oxidation pathway $(17,72)$. Thus, mitochondria and peroxisomes are metabolically interconnected and there is evidence that reactions in one organelle may affect the other and vice versa (73). Mitochondrial fatty acid oxidation is linked to gluconeogenesis and ketogenesis, pathways that allow the liver to release glucose and ketone bodies in the blood to provide fuels in the fasted state. Previous studies have confirmed that the amount of (acyl-)carnitines present will affect these processes; an increase in (acyl-)carnitines in the liver will increase fatty acid oxidation, gluconeogenesis, and ketogenesis $(74,75)$. Because Nudt7 and Crot compete for MCA-CoAs as substrates, the activity of Nudt7 could potentially affect the flux of MCA-carnitines into the mitochondria. 


\section{Objective}

CoA is a key regulator of energy metabolism and cell function. Because CoA is such a critical cofactor, maintaining a homeostatic level is essential to keep the metabolic processes it is involved with at optimal rates. It has been established that synthesis is an important mechanism to control CoA levels; however, degradation is another mechanism that may rapidly adjust CoA levels to the metabolic state. The objective of this study is to determine the in vitro biochemical characteristics of the CoA-degrading enzyme Nudt7 and its role in liver metabolism. To fulfill this objective, we will use a two-pronged approach of in vitro biochemistry and in vivo mouse studies. Characterizing the in vitro biochemical features such as substrate specificity, regulation, and key catalytic domains/residues will provide insight into the role of Nudt7 in the liver. Comparing the biochemical characteristics of Nudt7 to Nudt19, the CoA-degrading enzyme expressed in kidneys, may help answer the question of why two isoforms exist in the same subcellular compartment but different organs. Determining the structure of Nudt7 and Nudt19 with modeling and/or crystallography will provide additional information about the substrate binding domains and the fold of a unique insertion found in Nudt19. Overexpressing Nudt7 in the livers of mice will provide an in vivo approach to analyzing the involvement of Nudt7 in regulating hepatic CoA levels and thus, hepatic metabolic pathways. Analyzing the levels of total CoA in the livers of mice overexpressing Nudt7 will determine in decrease in the enzyme activity upon fasting contributes to the significant increase in liver CoA. Analyzing changes in the composition of the acyl-CoA pool and the metabolome of livers overexpressing Nudt7 will identify the in vivo substrates and potential metabolic pathways that Nudt7 regulates in the liver. 


\section{$\underline{\text { References }}$}

1. Robishaw, J. D., and Neely, J. R. (1985) Coenzyme A metabolism. American Journal of Physiology-Endocrinology And Metabolism 248

2. Leonardi, R., Zhang, Y.-M. M., Rock, C. O., and Jackowski, S. (2004) Coenzyme A: back in action. Progress in lipid research 44, 125-153

3. Lipmann, F., Kaplan, N. O., Novelli, G. D., Tuttle, L. C., and Guirard, B. M. (1950) Isolation of coenzyme A. The Journal of biological chemistry 186, 235-243

4. Pierpoint, W. S., and Hughes, D. E. (1954) The synthesis of coenzyme A by Lactobacillus arabinosus 17-5. Biochemical Journal 56, 130-135

5. Brass, E. P. (1994) Overview of coenzyme A metabolism and its role in cellular toxicity. Chemicobiological interactions 90, 203-214

6. Bandyopadhyay, G. K., Yu, J. G., Ofrecio, J., and Olefsky, J. M. (2006) Increased malonyl-CoA levels in muscle from obese and type 2 diabetic subjects lead to decreased fatty acid oxidation and increased lipogenesis; thiazolidinedione treatment reverses these defects. Diabetes 55, 2277-2285

7. Jackowski, S., and Leonardi, R. (2014) Deregulated coenzyme A, loss of metabolic flexibility and diabetes. . Biochemical Society transactions. 42, 1118-1122

8. Thompson, M., and Mayer, J. (1962) Coenzyme A and acetylation in various experimental obesities. The American journal of physiology 202, 1005-1010

9. Black, P. N., Faergeman, N. J., and DiRusso, C. C. (2000) Long-chain acyl-CoA-dependent regulation of gene expression in bacteria, yeast and mammals. The Journal of nutrition 130

10. Zhang, Y.-M. M., Chohnan, S., Virga, K. G., Stevens, R. D., Ilkayeva, O. R., Wenner, B. R., Bain, J. R., Newgard, C. B., Lee, R. E., Rock, C. O., and Jackowski, S. C. (2007) Chemical knockout of pantothenate kinase reveals the metabolic and genetic program responsible for hepatic coenzyme A homeostasis. Chemistry \& biology 14, 291-302

11. Horie, S., Isobe, M., and Suga, T. (1986) Changes in CoA pools in hepatic peroxisomes of the rat under various conditions. Journal of biochemistry 99, 1345-1352

12. Van Broekhoven, A., Peeters, M. C., Debeer, L. J., and Mannaerts, G. P. (1981) Subcellular distribution of coenzyme A: evidence for a separate coenzyme A pool in peroxisomes. Biochemical and biophysical research communications 100, 305-312

13. Sabari, B. R., Zhang, D., Allis, C. D., and Zhao, Y. (2017) Metabolic regulation of gene expression through histone acylations. Nat Rev Mol Cell Biol 18, 90-101

14. Li, Q. L., Yamamoto, N., Inoue, A., and Morisawa, S. (1990) Fatty acyl-CoAs are potent inhibitors of the nuclear thyroid hormone receptor in vitro. Journal of biochemistry 107, 699-702

15. Li, Q., Yamamoto, N., Morisawa, S., and Inoue, A. (1993) Fatty acyl-CoA binding activity of the nuclear thyroid hormone receptor. Journal of cellular biochemistry 51, 458-464

16. Hertz, R., Magenheim, J., Berman, I., and Bar-Tana, J. (1998) Fatty acyl-CoA thioesters are ligands of hepatic nuclear factor-4alpha. Nature 392, 512-516

17. Ramsay, R. R., and Zammit, V. A. (2004) Carnitine acyltransferases and their influence on CoA pools in health and disease. Carnitine acyltransferases and their influence on CoA pools in health and disease

18. Jonas, M., Pehar, M., and Puglielli, L. (2010) AT-1 is the ER membrane acetyl-CoA transporter and is essential for cell viability. $J$ Cell Sci 123, 3378-3388

19. Peng, Y., Li, M., Clarkson, B. D., Pehar, M., Lao, P. J., Hillmer, A. T., Barnhart, T. E., Christian, B. T., Mitchell, H. A., Bendlin, B. B., Sandor, M., and Puglielli, L. (2014) Deficient Import of Acetyl-CoA into the ER Lumen Causes Neurodegeneration and Propensity to Infections, Inflammation, and Cancer. The Journal of Neuroscience 34, 6772-6789

20. Jackowski, S., and Rock, C. C. (1981) Regulation of coenzyme A biosynthesis. Journal of bacteriology 148, 926-932 
21. Rock, C. O., Calder, R. B., Karim, M. A., and Jackowski, S. (2000) Pantothenate kinase regulation of the intracellular concentration of coenzyme A. The Journal of biological chemistry 275, 13771383

22. Vallari, D. S., Jackowski, S., and Rock, C. O. (1987) Regulation of pantothenate kinase by coenzyme A and its thioesters. The Journal of biological chemistry 262, 2468-2471

23. Alfonso-Pecchio, A., Garcia, M., Leonardi, R., and Jackowski, S. (2012) Compartmentalization of mammalian pantothenate kinases. PloS one 7

24. Rock, C. O., Karim, M. A., Zhang, Y.-M., and Jackowski, S. (2002) The murine pantothenate kinase (Pank1) gene encodes two differentially regulated pantothenate kinase isozymes. Gene 291, 35-43

25. Hörtnagel, K., Prokisch, H., and Meitinger, T. (2003) An isoform of hPANK2, deficient in pantothenate kinase-associated neurodegeneration, localizes to mitochondria. Human Molecular Genetics 12, 321-327

26. Leonardi, R., Zhang, Y.-M., Lykidis, A., Rock, C. O., and Jackowski, S. (2007) Localization and regulation of mouse pantothenate kinase 2. FEBS Letters 581, 4639-4644

27. Zhang, Y.-M., Rock, C. O., and Jackowski, S. (2005) Feedback Regulation of Murine Pantothenate Kinase 3 by Coenzyme A and Coenzyme A Thioesters. Journal of Biological Chemistry 280, 32594-32601

28. Smith, C. M. (1978) The effect of metabolic state on incorportion of [14C] pantothenate into CoA in rat liver and heart. The Journal of nutrition 108, 863-873

29. Reibel, D. K., Wyse, B. W., Berkich, D. A., and Neely, J. R. (1981) Regulation of coenzyme A synthesis in heart muscle: effects of diabetes and fasting. The American journal of physiology 240, 11

30. Robishaw, J. D., Berkich, D., and Neely, J. R. (1982) Rate-limiting step and control of coenzyme A synthesis in cardiac muscle. The Journal of biological chemistry 257, 10967-10972

31. Smith, C. M., and Savage, R. C. (1980) Regulation of coenzyme A biosynthesis by glucagon and glucocorticoid in adult rat liver parenchymal cells. Biochemical Journal 188, 175-184

32. Leonardi, R., Rehg, J. E., Rock, C. O., and Jackowski, S. (2010) Pantothenate Kinase 1 Is Required to Support the Metabolic Transition from the Fed to the Fasted State. PloS one 5

33. Gregory, A., and Hayflick, S. (2002 Aug 13 [Updated 2013 Jan 31]) Pantothenate KinaseAssociated Neurodegeneration. GeneReviews ${ }^{\circledR}$ [Internet].Seattle (WA): University of Washington, Seattle; 1993-2016.

34. Kuo, Y.-M. M., Duncan, J. L., Westaway, S. K., Yang, H., Nune, G., Xu, E. Y., Hayflick, S. J., and Gitschier, J. C. (2005) Deficiency of pantothenate kinase 2 (Pank2) in mice leads to retinal degeneration and azoospermia. Human Molecular Genetics 14, 49-57

35. Garcia, M., Leonardi, R., Zhang, Y. M., Rehg, J. E., and Jackowski, S. (2012) Germline deletion of pantothenate kinases 1 and 2 reveals the key roles for CoA in postnatal metabolism. PloS one 7, e40871

36. Lin, C.-C. C., Kitagawa, M., Tang, X., Hou, M.-H. H., Wu, J., Qu, D. C., Srinivas, V., Liu, X., Thompson, J. W., Mathey-Prevot, B., Yao, T.-P. P., Lee, S. H., and Chi, J.-T. T. (2018) CoA synthase regulates mitotic fidelity via CBP-mediated acetylation. Nature communications $\mathbf{9}, 1039$

37. Leonardi, R., Rock, C. O., and Jackowski, S. (2014) Pank1 deletion in leptin-deficient mice reduces hyperglycaemia and hyperinsulinaemia and modifies global metabolism without affecting insulin resistance. Diabetologia 57, 1466-1475

38. Corbin, D. R., Rehg, J. E., Shepherd, D. L., Stoilov, P., Percifield, R. J., Horner, L., Frase, S., Zhang, Y.-M. M., Rock, C. O., Hollander, J. M., Jackowski, S., and Leonardi, R. (2017) Excess coenzyme A reduces skeletal muscle performance and strength in mice overexpressing human PANK2. Molecular genetics and metabolism

39. Brass, E. P., Tahiliani, A. G., Allen, R. H., and Stabler, S. P. (1990) Coenzyme A metabolism in vitamin B-12-deficient rats. The Journal of nutrition 120, 290-297 
40. Bremer, J., Wojtczak, A., and Skrede, S. (1972) The Leakage and Destruction of CoA in Isolated Mitochondria. European Journal of Biochemistry 25

41. Skrede, S. (1973) The Degradation of CoA: Subcellular Localization and Kinetic Properties of CoA-and Dephospho-CoA Pyrophosphatase. European Journal of Biochemistry 38

42. Stipanuk, M. H., Dominy, J. E., Lee, J.-I., and Coloso, R. M. (2006) Mammalian cysteine metabolism: new insights into regulation of cysteine metabolism. The Journal of nutrition 136

43. Bessman, M. J., Frick, D. N., and O'Handley, S. F. (1996) The MutT proteins or "Nudix" hydrolases, a family of versatile, widely distributed, "housecleaning" enzymes. The Journal of biological chemistry 271, 25059-25062

44. McLennan, A. G. (2006) The Nudix hydrolase superfamily. Cellular and molecular life sciences : CMLS 63, 123-143

45. Cartwright, J. L., Gasmi, L., Spiller, D. G., and McLennan, A. G. (2000) The Saccharomyces cerevisiae PCD1 gene encodes a peroxisomal nudix hydrolase active toward coenzyme A and its derivatives. The Journal of biological chemistry 275, 32925-32930

46. AbdelRaheim, S. R., and McLennan, A. G. (2002) The Caenorhabditis elegans Y87G2A.14 Nudix hydrolase is a peroxisomal coenzyme A diphosphatase. BMC biochemistry 3, 5

47. Xu, W., Shen, J., Dunn, C. A., Desai, S., and Bessman, M. J. (2001) The Nudix hydrolases of Deinococcus radiodurans. Molecular microbiology 39, 286-290

48. Gasmi, L., and McLennan, A. (2001) The mouse Nudt7 gene encodes a peroxisomal nudix hydrolase specific for coenzyme A and its derivatives. The Biochemical journal 357, 33-38

49. Ofman, R., Speijer, D., Leen, R., and Wanders, R. J. C. (2006) Proteomic analysis of mouse kidney peroxisomes: identification of RP2p as a peroxisomal nudix hydrolase with acyl-CoA diphosphatase activity. The Biochemical journal 393, 537-543

50. Kang, L.-W. W., Gabelli, S. B., Bianchet, M. A., Xu, W. L., Bessman, M. J., and Amzel, L. C. (2003) Structure of a coenzyme A pyrophosphatase from Deinococcus radiodurans: a member of the Nudix family. Journal of bacteriology 185, 4110-4118

51. Crystal structure of Human Peroxisomal coenzyme A diphosphatase NUDT7. TO BE PUBLISHED

52. Reilly, S.-J., Tillander, V., Ofman, R., Alexson, S., and Hunt, M. (2008) The nudix hydrolase 7 is an Acyl-CoA diphosphatase involved in regulating peroxisomal coenzyme A homeostasis. Journal of biochemistry 144, 655-663

53. Tillander, V., Alexson, S. E. H., and Cohen, D. E. (2017) Deactivating Fatty Acids: Acyl-CoA Thioesterase-Mediated Control of Lipid Metabolism. Trends Endocrinol Metab 28, 473-484

54. Hunt, M., Tillander, V., and Alexson, S. (2014) Regulation of peroxisomal lipid metabolism: the role of acyl-CoA and coenzyme A metabolizing enzymes. Biochimie 98, 45-55

55. Hunt, M. C., Siponen, M. I., and Alexson, S. E. (2012) The emerging role of acyl-CoA thioesterases and acyltransferases in regulating peroxisomal lipid metabolism. Biochimica et biophysica acta 1822, $1397-1410$

56. Westin, M. A., Hunt, M. C., and Alexson, S. E. (2005) The identification of a succinyl-CoA thioesterase suggests a novel pathway for succinate production in peroxisomes. J Biol Chem $\mathbf{2 8 0}$, 38125-38132

57. Westin, M. A., Alexson, S. E., and Hunt, M. C. (2004) Molecular cloning and characterization of two mouse peroxisome proliferator-activated receptor alpha (PPARalpha)-regulated peroxisomal acyl-CoA thioesterases. J Biol Chem 279, 21841-21848

58. Hunt, M. C., Rautanen, A., Westin, M. A., Svensson, L. T., and Alexson, S. E. (2006) Analysis of the mouse and human acyl-CoA thioesterase (ACOT) gene clusters shows that convergent, functional evolution results in a reduced number of human peroxisomal ACOTs. FASEB $J \mathbf{2 0}$, 1855-1864

59. Ferdinandusse, S., and Houten, S. M. (2006) Peroxisomes and bile acid biosynthesis. Biochim Biophys Acta 1763, 1427-1440

60. Russell, D. W. (2003) The enzymes, regulation, and genetics of bile acid synthesis. Annu Rev Biochem 72, 137-174 
61. Watkins, P. A., and Ellis, J. M. (2012) Peroxisomal acyl-CoA synthetases. Biochimica et biophysica acta 1822, 1411-1420

62. Baes, M., and Van Veldhoven, P. P. (2016) Hepatic dysfunction in peroxisomal disorders. Biochimica et biophysica acta 1863, 956-970

63. Waterham, H. R., Ferdinandusse, S., and Wanders, R. J. A. (2016) Human disorders of peroxisome metabolism and biogenesis. Biochimica et Biophysica Acta (BBA) - Molecular Cell Research 1863, 922-933

64. Seedorf, U., Raabe, M., Ellinghaus, P., Kannenberg, F., Fobker, M., Engel, T., Denis, S., Wouters, F., Wirtz, K. W., Wanders, R. J., Maeda, N., and Assmann, G. (1998) Defective peroxisomal catabolism of branched fatty acyl coenzyme A in mice lacking the sterol carrier protein-2/sterol carrier protein-x gene function. Genes Dev 12, 1189-1201

65. Kannenberg, F., Ellinghaus, P., Assmann, G., and Seedorf, U. (1999) Aberrant oxidation of the cholesterol side chain in bile acid synthesis of sterol carrier protein-2/sterol carrier protein-x knockout mice. The Journal of biological chemistry 274, 35455-35460

66. Herrema, H., Meissner, M., van Dijk, T. H., Brufau, G., Boverhof, R., Oosterveer, M. H., Reijngoud, D.-J. J., MÃ¹/4ller, M., Stellaard, F., Groen, A. K., and Kuipers, F. (2010) Bile salt sequestration induces hepatic de novo lipogenesis through farnesoid $\mathrm{X}$ receptor- and liver $\mathrm{X}$ receptor alpha-controlled metabolic pathways in mice. Hepatology (Baltimore, Md.) 51, 806-816

67. Reddy, J. K., and Hashimoto, T. (2001) Peroxisomal $\beta$-oxidation and peroxisome proliferatoractivated receptor $\alpha$ : an adaptive metabolic system. Annual review of nutrition 21, 193-230

68. Fournier, B., Saudubray, J. M., Benichou, B., Lyonnet, S., Munnich, A., Clevers, H., and Poll-The, B. T. (1994) Large deletion of the peroxisomal acyl-CoA oxidase gene in pseudoneonatal adrenoleukodystrophy. The Journal of clinical investigation 94, 526-531

69. Varanasi, U., Chu, R., Chu, S., Espinosa, R., LeBeau, M. M., and Reddy, J. K. (1994) Isolation of the human peroxisomal acyl-CoA oxidase gene: organization, promoter analysis, and chromosomal localization. Proceedings of the National Academy of Sciences 91, 3107-3111

70. $\quad$ Fan, C.-Y., Pan, J. I. E., Chu, R., Lee, D., Kluckman, K. D., Usuda, N., Singh, I., Yeldandi, A. V., Rao, S. M., Maeda, N., and Reddy, J. K. (1996) Targeted Disruption of the Peroxisomal Fatty AcylCoA Oxidase Gene: Generation of a Mouse Model of Pseudoneonatal Adrenoleukodystrophya. Annals of the New York Academy of Sciences 804, 530-541

71. Fan, C. Y., Pan, J., Chu, R., Lee, D., and Kluckman, K. D. (1996) Hepatocellular and hepatic peroxisomal alterations in mice with a disrupted peroxisomal fatty acyl-coenzyme A oxidase gene. Journal of Biological Chemistry 271, 24698-24710

72. Lodhi, I. J., and Semenkovich, C. F. (2014) Peroxisomes: a nexus for lipid metabolism and cellular signaling. Cell metabolism 19, 380-392

73. Camões, F., Bonekamp, N. A., Delille, H. K., and Schrader, M. (2008) Organelle dynamics and dysfunction: A closer link between peroxisomes and mitochondria. Journal of Inherited Metabolic Disease 32, 163-180

74. McGarry, J. D., Robles-Valdes, C., and Foster, D. C. (1975) Role of carnitine in hepatic ketogenesis. Proceedings of the National Academy of Sciences of the United States of America 72, 4385-4388

75. Delisle, G., and Fritz, I. C. (1967) Interrelations between hepatic fatty acid oxidation and gluconeogenesis: a possible regulatory role of carnitine palmityltransferase. Proceedings of the National Academy of Sciences of the United States of America 58, 790-797 


\section{Chapter II: Characterization of the biochemical and structural properties of Nudt7 and Nudt19}

Shumar, S. A., Kerr, E. W., Geldenhuys, W. J., Montgomery, G. E., Fagone, P., Thirawatananond, P., Saavedra, H., Gabelli, S. B., \& Leonardi, R. (2018). Nudt19 is a renal CoA diphosphohydrolase with biochemical and regulatory properties that are distinct from the hepatic Nudt7 isoform. Journal of

Biological Chemistry, 293(11), 4134-4148.

Author Contributions

SAS, RL, EWK, WJG and SBG wrote the manuscript; SAS, RL and SBG designed the study; SAS purified all the enzymes and performed all the enzymatic assays and CoA measurements; SAS and GM generated the Nudt7 and Nudt19 mutants; EWK conducted the immunofluorescence studies; WJG generated the homology model of Nudt19; PF analyzed Nudt7, Nudt19 and the chimeras by analytical gel filtration; PT, HSE and SBG performed the ITC studies. All authors analyzed the results and approved the final version of the manuscript.

This research was originally published in the Journal of Biological Chemistry. Shumar, S. A., Kerr, E. W., Geldenhuys, W. J., Montgomery, G. E., Fagone, P., Thirawatananond, P., Saavedra, H., Gabelli, S. B., \& Leonardi, R. Nudt19 is a renal CoA diphosphohydrolase with biochemical and regulatory properties that are distinct from the hepatic Nudt7 isoform. J. Biol. Chem. 2018; 293: 4134-4148.

(c) The American Society for Biochemistry and Molecular Biology 


\section{$\underline{\text { Abstract }}$}

CoA is the major acyl carrier in mammals and a key cofactor in energy metabolism. Dynamic regulation of CoA in different tissues and organs supports metabolic flexibility. Two mammalian Nudix hydrolases, Nudt19 and Nudt7, degrade CoA in vitro. Nudt19 and Nudt7 possess conserved Nudix and CoA signature sequences, and specifically hydrolyze the diphosphate bond of free CoA and acyl-CoAs to form 3',5'-ADP and 4'-(acyl)phosphopantetheine. Limited information is available on these enzymes, but the relatively high abundance of Nudt19 and Nudt7 mRNA in the kidney and liver, respectively, suggests that they play distinct roles in the regulation of CoA levels in these organs.

Here, we analyzed Nudt19-- mice and found that deletion of Nudt19 elevates kidney CoA levels in mice fed ad libitum, indicating that Nudt19 contributes to the regulation of CoA in vivo. Unlike what observed for the regulation of Nudt7 in the liver, Nudt19 transcript and protein levels in the kidney did not differ between fed and fasted states. Instead, we identified chenodeoxycholic acid (CDCA) as a specific Nudt19 inhibitor that competed with CoA for Nudt19 binding but did not bind to Nudt7. Exchange of the Nudix and CoA signature motifs between the two isoforms dramatically decreased their $\mathrm{k}_{\text {cat. }}$ Furthermore, substitutions of conserved residues within these motifs identified amino acids playing different roles in CoA binding and hydrolysis in Nudt19 and Nudt7.

Our results reveal that the kidney and liver each possesses a distinct peroxisomal CoA diphosphohydrolase.

\section{Introduction}

Coenzyme A (CoA) is an obligate cofactor utilized as an acyl carrier in hundreds of metabolic reactions. Numerous CoA thioesters are also involved in the post-translational modification of histones and thousands of other proteins (1-5). Three major subcellular CoA pools are found in the cytosol, mitochondria, and peroxisomes to support specific metabolic pathways $(6,7)$. These include, among others, fatty acid synthesis in the cytosol, the TCA cycle and oxidation of long-chain and medium-chain fatty acids in the mitochondria, and bile acid conjugation and oxidation of very long-chain and branched-chain fatty 
acids in the peroxisomes. A dedicated pool of acetyl-CoA is also found in the endoplasmic reticulum where it is involved in protein quality control and autophagy (8), while nuclear acyl-CoAs, which freely equilibrate with the cytosolic pool across the nuclear pores, contributes to the regulation of gene expression (5).

Tight control over the concentration of CoA in different organs is essential to maintain normal metabolism and organ function. Indeed, elevated CoA levels in the liver of diabetic mice promote excessive gluconeogenesis and hyperglycemia (9), while a supraphysiological concentration of CoA in skeletal muscle leads to decreased ATP levels and exercise performance (10). Adverse consequences are also associated with lower than normal CoA levels. Decreased synthesis of this cofactor in the nervous system is associated with a rare neurological disorder in humans $(11,12)$, while decreased synthesis of CoA in the liver leads to fasting hypoglycemia and hepatic triglyceride accumulation (13). Dynamic regulation of CoA within the normal physiological range is important to support the metabolic reprogramming that underlies the capacity to respond to changes in the metabolic state. For example, the increase in CoA that characterizes the fed-to-fasted transition in the liver is driven by the activation of the biosynthetic pathway and is required to sustain the high rates of hepatic fatty acid oxidation and gluconeogenesis under fasting conditions $(13,14)$. On the other hand, the net decrease in the concentration of CoA observed upon refeeding after a fast requires both inhibition of de novo CoA synthesis and active degradation of the cofactor accumulated in the fasted state.

The existence of a CoA-degradation pathway in the liver and kidneys is supported by the fact that mice treated with an inhibitor of the CoA biosynthetic pathway, exhibit a dramatic reduction in hepatic and renal CoA, indicating rapid CoA turnover in these tissues (15). Liver and kidneys contain high transcript levels of two Nudix hydrolases, Nudt7 and Nudt19, respectively, which exhibit CoA diphosphohydrolase activity in vitro $(16,17)$. Nudix hydrolases hydrolyze the diphosphate bond of a variety of substrates consisting of a nucleoside diphosphate linked to another moiety, $\underline{x}(18,19)$. All members of the Nudix superfamily contain the conserved Nudix box motif $\mathrm{G}_{1}{ }^{\mathrm{N}}[5 \mathrm{X}] \mathrm{E}_{7}{ }^{\mathrm{N}}[7 \mathrm{X}] \mathrm{R}_{15}{ }^{\mathrm{N}} \mathrm{E}_{16}{ }^{\mathrm{N}} \mathrm{XXE}_{19}{ }^{\mathrm{N}} \mathrm{E}_{20}{ }^{\mathrm{N}} \mathrm{XG}_{22}{ }^{\mathrm{N}} \mathrm{U}$ (where $\mathrm{U}$ is a hydrophobic residue, $\mathrm{X}$ is any residue and superscript $\mathrm{N}$ denotes the position of a residue of the signature motif within a specific protein sequence) (20,21). Additionally, Nudix hydrolases that 
specifically hydrolyze CoA also contain a putative CoA binding motif, (L/M)(L/F)TXR(S/A)[3X](R/K)[3X]G[3X]FPGG (PROSITE accession number PS01293, formerly UPF0035), upstream of the Nudix box motif (22). The crystal structure of human NUDT7 has recently been released (PDB ID: 5T3P) (23). Similar to the structure of the bacterial homolog from D. radiodurans (24), the crystal structure of the human enzyme does not contain CoA bound. Thus, the contribution of the CoA motif to CoA binding and the organization of the CoA binding site in these enzymes are currently unknown.

Independent studies and approaches have demonstrated the localization of Nudt7 in the peroxisomes $(17,25)$. Nudt7 mRNA and protein levels in the liver are regulated by the nutritional state and are inversely correlated with the tissue concentration of CoA $(9,26)$. Additionally, overexpression of a catalytically active, cytosolic form of Nudt7 has also recently been shown to cause a decrease in CoA levels in vivo (25).

Significantly less is known about Nudt19. Nudt19 exhibits modest sequence similarity to Nudt7 (about 30\%), is larger than a typical Nudix hydrolase, including Nudt7, and contains a unique 45-49 amino acid insertion of unknown function within its Nudix box (16). This enzyme, also known as RP2p, was originally reported to be a kidney protein whose transcript levels were robustly upregulated by androgens (27). Later, recombinant Nudt19 was shown to specifically hydrolyze CoA species (16), although modest activity against capped RNA has also been detected (28). Based on proteomics studies, Nudt19 localization has been reported in both peroxisomes and mitochondria $(16,29)$; however it is currently unclear whether the enzyme has dual localization or exclusively resides in one of the two subcellular compartments, and which one. Given the presence of dedicated CoA pools and complementary metabolic processes in peroxisomes and mitochondria, this distinction is important to gain insight into the physiological role of Nudt19, even in relation to the other mammalian isoform, Nudt7. Indeed, it is presently unclear whether Nudt7 and Nudt19, which seem to be differentially expressed in liver and kidneys, differ in key aspects of their function. 
We have investigated the effect of Nudt19 deletion on CoA levels, the localization of this enzyme in intact cells, and its regulation by selected metabolites. Additionally, we used a combination of computational modeling, substrate docking and site directed mutagenesis to identify residues involved in CoA binding and hydrolysis in Nudt19, and analyzed the role of equivalent residues in Nudt7. We show that Nudt19 is a peroxisomal enzyme that contributes to the regulation of kidney CoA levels in vivo and provide evidence supporting the conclusion that the CoA binding sites of Nudt19 and Nudt7 are different.

\section{$\underline{\text { Material and methods }}$}

\section{Materials}

Reagents were purchased from the following suppliers: oligonucleotides, Lipofectamine 2000, cell culture reagents Alexa Flour 555 and HRP-conjugated goat anti-rabbit IgG from ThermoFisher Scientific; restriction enzymes, DNA polymerases and the Q5 ${ }^{\circledR}$ Site-Directed Mutagenesis Kit from New England Biolabs; HEK 293 cells from the American Type Culture Collection, and the GAPDH antibody from Cell Signaling. The PMP70 antibody was purchased from Sigma-Aldrich. The antibody against the full-length mouse Nudt19 was raised in rabbit and purified by antigen affinity chromatography. The Nudt7 antibody was generated as previously described (9). mBB-CoA was synthesized from free CoA and monobromobimane (Echelon Biosciences) as described by Shimada et al. (30). The conversion of free CoA to mBB-CoA was $>99.0 \%$, as estimated by HPLC. All other reagents were of analytical grade or better and were purchased from Sigma-Aldrich or Fisher Scientific, unless otherwise stated.

\section{Animal Studies}

Nudt19 ${ }^{\text {tm1(KOMP)Vlcg }}$ embryos, obtained by replacing the Nudt19 gene with a ZEN-UB1 cassette harboring the lacZ reporter and neo resistance gene, were purchased from the Knockout Mouse Project (KOMP) repository and implanted into pseudopregnant foster mothers by the Transgenic Animal Core Facility at West Virginia University. The progeny was screened for the deletion of the Nudt19 gene by multiplex PCR analysis using the Accustart II PCR Genotyping Kit (QuantaBio) and the following KOMP primers: $\quad$ Reg-NeoF $\quad$ (GCAGCCTCTGTTCCACATACACTTCA), Reg-Nudt19-R 
(AAGGACTCAACTCTCACCCTAAGCG). The Nudt19 knockout allele yielded a product of 466 bp. Although smaller than the size of 584 bp predicted by the KOMP repository, this 466 bp amplicon contained the expected 3' end of the ZEN-UB1 cassette (124 bases) and a sequence corresponding to the genomic region downstream of the Nudt19 gene (342 bp), as confirmed by sequencing. The wild type allele yielded a product of the expected size of 185 bp. Homozygous Nudt19-/ mice and wild-type C57BL6/N littermate controls were obtained through heterozygous x heterozygous breeding. Mice were fed a standard chow diet (Tekland 2018S) and maintained at a room temperature of $72.0 \pm 0.3^{\circ} \mathrm{F}$, room humidity of $40 \% \pm 2 \%$, and a 12-h light, 12-h dark cycle, with the dark cycle starting at 18:00 h. For experiments that required fasting, mice were transferred to clean cages containing floor grids, and food was removed for the indicated time. All studies were conducted on 10-14 week-old male mice and were approved by the Institutional Animal Care and Use Committees of West Virginia University.

CoA Analysis, Immunoblotting and RT-PCR

The concentration of total CoA (free CoA plus CoA thioesters) in kidney homogenates was determined after conversion of the cofactor to the monobromobimane derivative, as previously described (25). For western blot analysis, flash frozen tissues were homogenized in ice-cold radioimmunoprecipitation assay (RIPA) buffer supplemented with protease inhibitors (Biotool), and centrifuged at $10,000 \mathrm{x} g$ for $10 \mathrm{~min}$ at $4^{\circ} \mathrm{C}$. Proteins were fractionated on $4-12 \%$ bis-Tris polyacrylamide gels and transferred onto PVDF membranes. The GAPDH antibody was used at a 1:3000 dilution; the Nudt7 and Nudt19 antibodies were used at a 1:10,000 dilution. Bound primary antibodies were detected by chemiluminescence with HRP-conjugated goat anti-rabbit IgG at a 1:45,000 dilution. RNA was isolated from flash frozen tissue as previously described (31). Following the removal of genomic DNA with Turbo DNAse-free kit (Thermo Fisher Scientific), Nudt19 mRNA levels were quantified in triplicate by RT-PCR using the Quantitect SYBR Green RT-PCR kit (Qiagen) and the primers previously reported (31). The 


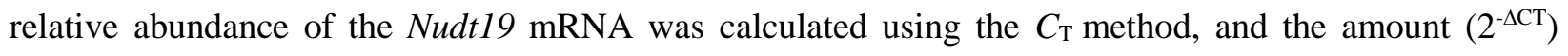
reported relative to the mRNA of beta-glucuronidase (GusB).

Plasmid Construction and Mutagenesis

Wild type mouse Nudt7 and Nudt19 were expressed as N-terminal hexahistine-tagged proteins from plasmids pKM204 (25) and pKM249, respectively. Plasmid pKM249 was a kind gift from Dr. Suzanne Jackowski, St. Jude Children's Research Hospital. The above plasmids were mutagenized with the Q $5{ }^{\circledR}$ Site-Directed Mutagenesis Kit to generate single point Nudt7 and Nudt19 mutants, and a Nudt19 deletion mutant lacking amino acids 58-103. Insertion of the correct mutations was confirmed by sequencing analysis by the Genomics Core Facility at West Virginia University. Sequences encoding Nudt7 and Nudt19 chimeras were custom synthesized (ThermoFisher Scientific) to contain NdeI and NotI restriction sites at the 5' and 3' ends, respectively, codon-optimized for expression in E. coli and subcloned into pET-28a(+) (Millipore). For the expression and localization of mouse Nudt19 in HEK 293 cells, custom pcDNA3.1(+)-derived constructs (GenScript) were assembled to express Nudt19 proteins, codonoptimized for expression in human cells, with $\mathrm{N}$ - or C-terminal GFP tags.

Protein Purification, Analytical Gel Filtration Chromatography and Isothermal Titration Calorimetry (ITC)

All Nudt7 and Nudt19 proteins with N-terminal hexahistidine tags were expressed in BL21 CodonPlus (DE3)-RIPL (Agilent Technologies) cells, following induction with $1 \mathrm{mM}$ isopropyl $\beta$-D-1thiogalactopyranoside and growth for $18-20 \mathrm{~h}$ at $18-25^{\circ} \mathrm{C}$. For enzymatic assay, the enzymes were purified as previously described (25). Fractions containing $~ 90 \%$ pure enzymes were combined and dialyzed overnight at $4^{\circ} \mathrm{C}$ in buffer containing $20 \mathrm{mM}$ Tris-HCl, pH 8.0, $300 \mathrm{mM} \mathrm{NaCl}, 1 \mathrm{mM}$ EDTA and $1 \mathrm{mM}$ dithiothreitol. Glycerol was added to a final concentration of $50 \%$ and proteins were stored at $-20^{\circ} \mathrm{C}$. Protein samples analyzed by analytical gel filtration chromatography were loaded (50 $\mu$ l) onto a Superdex 75 10/300 GL column and eluted at $0.3 \mathrm{ml} / \mathrm{min}$ with $50 \mathrm{mM}$ Tris- $\mathrm{HCl}, 150 \mathrm{mM} \mathrm{NaCl}$, pH 8.0. Protein elution was monitored by measuring the absorbance at $280 \mathrm{~nm}$. For ITC studies, the purification procedure 
included three chromatographic steps to increase the purity of the proteins to $>98 \%$, and the hexahistidine tag was removed. Briefly, cell pellets were lysed in $50 \mathrm{mM} \mathrm{Na} 2 \mathrm{HPO}_{4} / \mathrm{NaH}_{2} \mathrm{PO}_{4}, \mathrm{pH} 8.0,300 \mathrm{mM} \mathrm{NaCl}$, $10 \mathrm{mM}$ imidazole and centrifuged at 13,000 rpm for $45 \mathrm{~min}$ at $4^{\circ} \mathrm{C}$. The clarified supernatant was allowed to bind to the Ni-NTA resin for $2 \mathrm{~h}$ with stirring, before the suspension was transferred into a column. The resin-bound proteins were washed with $50 \mathrm{mM} \mathrm{Na} \mathrm{HPO}_{4} / \mathrm{NaH}_{2} \mathrm{PO}_{4}, \mathrm{pH}$ 8.0, $300 \mathrm{mM} \mathrm{NaCl}, 20 \mathrm{mM}$ imidazole, followed by elution with wash buffer containing 50-100 mM of imidazole. Protein-containing fractions were combined, dialyzed in buffer A (50 mM Na $2 \mathrm{HPO}_{4} / \mathrm{NaH}_{2} \mathrm{PO}_{4}, \mathrm{pH}$ 8.0, $200 \mathrm{mM} \mathrm{NaCl}$ ) and incubated with $0.04 \mathrm{U} / \mathrm{ml}$ thrombin (Sigma-Aldrich) overnight at room temperature to remove the hexahistine tag. The salt concentration of the protein samples was then lowered with an additional dialysis step in buffer $\mathrm{B}\left(50 \mathrm{mM} \mathrm{Na} \mathrm{HPO}_{4} / \mathrm{NaH}_{2} \mathrm{PO}_{4}, 50 \mathrm{mM} \mathrm{NaCl}\right)$, before applying the proteins onto a Resource15Q anion exchange column equilibrated in buffer B. Proteins were eluted with a 0-100\% gradient of buffer $\mathrm{C}\left(50 \mathrm{mM} \mathrm{Na} \mathrm{HPO}_{4} / \mathrm{NaH}_{2} \mathrm{PO}_{4}, \mathrm{pH} 8.0,500 \mathrm{mM} \mathrm{NaCl}\right)$, and the fractions containing the proteins of interested were pooled, dialyzed in buffer A and concentrated to $5 \mathrm{ml}$. The concentrated protein samples were subsequently loaded onto a HiLoad 26/60 Superdex 75 pg size exclusion column equilibrated in buffer A, eluted in the same buffer, concentrated and stored at $-80^{\circ} \mathrm{C}$.

Binding affinity of wild type Nudt7 and Nudt19 for CDCA was measured by ITC at $25^{\circ} \mathrm{C}$ using a VP-ITC instrument (Microcal Inc, Northhampton, MA) and $1.4 \mathrm{ml}$ of purified Nudt7 and Nudt19 dialyzed in ITC buffer (30 mM PIPES pH 7.5, $50 \mathrm{mM} \mathrm{NaCl,} 2 \mathrm{mM} \mathrm{MgCl}_{2}$ ) and diluted to $124 \mu \mathrm{M}$ and $55.5 \mu \mathrm{M}$, respectively. Protein solutions were degassed for at least 20 minutes. CDCA stock solutions were prepared in the same ITC buffer. After an initial $2 \mu$ injection of CDCA solution with a 240 second equilibration time, the compound was titrated in 30 injections of $10 \mu \mathrm{l}$ each, added at 300 second intervals. The duration of each injection was 12 seconds and the filter period 2 seconds. The stirring speed and Feedback Mode/gain option were set up to $300 \mathrm{rpm}$ and "High" respectively. The heat evolved at $25^{\circ} \mathrm{C}$ after each ligand injection was obtained by integration of the calorimetric signal after subtracting the average heat of dilution. Binding parameters were determined from titration binding curves using the MicroCal Data Analysis software (Malvern, Westborough, MA). 


\section{Enzymatic Assays}

The CoA diphosphohydrolase activity of the recombinant enzymes was assayed, in duplicate, as previously described (25), with minor modifications. The standard assay contained $10 \mathrm{ng}$ of recombinant Nudt7 or $30 \mathrm{ng}$ of Nudt19, $100 \mathrm{mM}$ Tris-HCl $\mathrm{pH}$ 8.0, $0.1 \mathrm{mg} / \mathrm{ml}$ gamma globulin, an optimized concentration of $\mathrm{MgCl}_{2}$, which was $4 \mathrm{mM}$ for Nudt7 or $10 \mathrm{mM}$ for Nudt19, and $250 \mu \mathrm{M}$ of free CoA or acyl-CoA substrates, in a total volume of $40 \mu \mathrm{l}$. The reactions were incubated at $37^{\circ} \mathrm{C}$ for $10 \mathrm{~min}$ and stopped by the addition of perchloric acid. Following neutralization with potassium carbonate, the reaction mixtures were diluted 5-fold with water, and the UV-visible 3',5'-ADP product was separated and quantified by high performance liquid chromatography (HPLC) coupled with UV detection on a 4.6 x 150 $\mathrm{mm}, 3 \mu \mathrm{m} \mathrm{C}-18$ column (Acclaim 120 ) kept at $35^{\circ} \mathrm{C}$. Elution was performed at $0.7 \mathrm{ml} / \mathrm{min}$ as follows: 0 $2.5 \mathrm{~min}, 100 \%$ buffer A (50 mM KH $\mathrm{PO}_{4}$ ); 2.5-9.0 min linear gradient to 50\% B (100\% acetonitrile); 9.010.0 min isocratic at $50 \% \mathrm{~B} ; 10.0-15.0$ min linear gradient from $50 \%$ B to $75 \%$ B; $15.0-19.0$ min isocratic at $75 \% \mathrm{~B} ; 19.0-32.0$ min return to $100 \% \mathrm{~A}$, and column equilibration. A standard curve of 3',5'-ADP (Sigma-Aldrich) subjected to the same reaction conditions was used to quantify the amount of product formed. For each mutant and chimeric enzyme, protein curves were generated to select concentrations within the activity linear range. The length of the assay was also extended to $2 \mathrm{~h}$ for mutants exhibiting marginal activity. The kinetic parameters $\mathrm{K}_{\mathrm{M}}, \mathrm{V}_{\max }$, and $\mathrm{k}_{\mathrm{cat}}$ were determined using free CoA as the substrate, with concentrations ranging from $31.25 \mu \mathrm{M}$ to $10 \mathrm{mM}$, and obtained from curves fitted to Michaelis-Menten equation using GraphPad Prism 6 (GraphPad Software).

To monitor the production of 3',5'-ADP in kidney extracts, flash-frozen tissue ( $50 \mathrm{mg})$ was homogenized in $750 \mu \mathrm{l}$ of $20 \mathrm{mM}$ Tris- $\mathrm{HCl} \mathrm{pH}$ 8.0. The homogenate was incubated on ice for $10 \mathrm{~min}$ and centrifuged at 20,000 $\mathrm{xg}$ for $10 \mathrm{~min}$. The supernatant was loaded on to a PD10 column equilibrated in 20 $\mathrm{mM}$ Tris-HCl pH 8.0, to remove the small molecules. The eluted proteins (50-200 $\mu \mathrm{g}$ ) were incubated with $250 \mu \mathrm{M}$ free CoA for $5 \mathrm{~min}$ at $37^{\circ} \mathrm{C}$. The reactions were then stopped and analyzed as described above. 


\section{Immunofluorescence}

HEK 293 cells were cultured at $37^{\circ} \mathrm{C}$ and $5 \% \mathrm{CO}_{2}$ in phenol red-free Opti-MEM I medium supplemented with 4\% FBS, $100 \mathrm{U} / \mathrm{ml}$ penicillin, $100 \mu \mathrm{g} / \mathrm{ml}$ streptomycin and $0.25 \mu \mathrm{g} / \mathrm{ml}$ amphotericin B. To localize Nudt19, cells were seeded on $12 \mathrm{~mm}$ coverslips at low density and grown in the same medium to about $80 \%$ confluence. Transfections were performed with $1.25 \mu \mathrm{g}$ of plasmid DNA using Lipofectamine 2000, as per manufacturer's instructions. Where indicated, cells were incubated with Mitotracker ${ }^{\circledR}$ Orange CMTMRos at a concentration of $25 \mathrm{nM}$ for $30 \mathrm{~min}$ at $37^{\circ} \mathrm{C}$. The cells were fixed with 4\% paraformaldehyde, and blocked in PBS containing 5\% FBS and 0.3\% Triton X-100 for $1 \mathrm{~h}$ at room temperature. The PMP70 antibody was used at a 1:1000 dilution in 1\% BSA, 0.3\% Triton-X100 for $1 \mathrm{~h}$ at room temperature. Following 10 washes with PBS, the cells were incubated for $1 \mathrm{~h}$ at room temperature with Alexa Flour 555 donkey anti-mouse IgG, used at a dilution of 1:1000 in 1\% BSA, 0.3\% Triton X-100. Slides were mounted using Prolong Gold Antifade with DAPI (ThermoFisher). Imaging experiments were performed in the West Virginia University Imaging Facilities using a Nikon A1R/N SIM-E confocal microscope. Image analysis was conducted using ImageJ.

\section{Molecular Modeling}

Homology models with bound $\mathrm{Mg}^{2+}$ were built using the software suite YASARA (32) (www.yasara.org). The models showing the expected orientation of $\mathrm{E}_{16}{ }^{\mathrm{N}}, \mathrm{E}_{19}{ }^{\mathrm{N}}$ and $\mathrm{E}_{20} \mathrm{~N}$ with respect to the $\mathrm{Mg}^{2+}$ ion were selected and prepared for docking studies with free CoA by adding hydrogens at $\mathrm{pH} 7.4$ in the modeling program MOE 2016 (www.chemcomp.com). After an induced fit docking step, the top five poses were evaluated for the correct relative orientation of the diphosphate moiety of free CoA, the bound $\mathrm{Mg}^{2+}$ and Nudt19 R34 or NUDT7 R60. Sequence alignments were obtained using Clustal W (33) and combined with secondary structure elements using ESPript 3 (34). The Nudt19 model and the crystal structures of human NUDT7 and the bacterial CoAse were aligned using Phenix Ensembler (35). 


\section{Statistical Analysis}

Unless otherwise stated, all data are reported as the mean with \pm the standard deviation. Statistical significance was calculated by unpaired two-tailed Student's t test using GraphPad Prism 6 (GraphPad Software).

\section{$\underline{\text { Results }}$}

\section{Nudt19 Regulates Kidney CoA Levels in Vivo}

Previous work has shown that Nudt19 hydrolyzes free CoA and acyl-CoAs to 3',5'-ADP and 4'(acyl)phosphopantetheine in vitro (16). We confirmed these findings by measuring the activity of recombinant Nudt19 against several acyl-CoAs and comparing it to the better characterized Nudt7 $(17,25)$ (Fig. 2.1A and 1B). We determined that the optimal concentration of $\mathrm{MgCl}_{2}$ for Nudt7 and Nudt19 was 4 and $10 \mathrm{mM}$, respectively, when free CoA was used as the substrate (data not shown). However, in experiments where we tested multiple acyl-CoAs, it was necessary to keep the concentration of $\mathrm{MgCl}_{2}$ at 4 mM for both Nudt7 and Nudt19 to prevent the precipitation of lauroyl- and steroyl-CoA. Under these conditions, the specific activity per pmole of Nudt19 was lower than that of Nudt7, but the enzyme readily hydrolyzed short and medium chain acyl-CoAs, malonyl- and succinyl-CoA, in addition to free CoA (Fig. 2.1A). Exceptions were acetyl-CoA and the synthetic monobromobimane (mBB)-CoA, which were excellent substrates for Nudt7, but not for Nudt19. Nudt19 also had no detectable hydrolytic activity against ATP, ADP, $\mathrm{NAD}^{+} \mathrm{NADH}, \mathrm{NADP}^{+}$, and NADPH (data not shown), confirming the specificity of this enzyme for CoA (16).

The mRNA levels of Nudt19 are highly abundant in mouse kidneys (16,36). To examine the distribution of this protein in mouse tissues, we generated a polyclonal antibody against the full-length mouse Nudt19. Consistent with the mRNA expression pattern, Nudt19 protein showed the highest abundance in the kidneys, with lower but still detectable levels in skeletal muscle and brain (Fig. 2.1C). This expression pattern was strikingly different from Nudt7, which was expressed at the highest levels in 
the liver and, to a significantly lower extent, in white and brown adipose tissue (Fig. 2.1C). The high expression level of Nudt19, combined with the minimally detectable Nudt7 protein in the kidneys (Fig. 2.1C), suggested that Nudt19 was the major of the two isoforms in these organs.

To determine whether Nudt19 regulated CoA levels in vivo, we obtained Nudt19 ${ }^{+/}$mice from the KOMP repository and bred them to generate $\mathrm{Nudt}_{19^{-/}}$mice and wild type littermate controls. The Nudt19${ }^{\text {^ }}$ mice were viable, fertile and outwardly normal. Successful deletion of the gene was verified by PCR analysis of tail biopsies (Fig. 2.2A), while western blot analysis confirmed the lack of Nudt19 protein expression (Fig. 2.2B). Additionally, enzymatic assays confirmed the lack of any residual formation of 3',5'-ADP in kidney homogenates obtained from $\mathrm{Nudt19}^{-/}$mice (Fig. 2.2C). The concentration of CoA was measured in the kidneys of $\mathrm{Nudt} 19^{-/}$and control mice in the fed state and following an overnight fast. In the fed state, the kidneys of the Nudt $19^{-/-}$mice exhibited a significant $20 \%$ increase in the concentration of CoA compared to the wild type mice (Fig. 2.2D). Fasting induced an increase in kidney CoA levels in both wild type and $\mathrm{Nudt}_{19^{-/}}$mice. Under these conditions, the concentration of CoA in the Nudt19\%kidneys tended to be higher than control mice, but the difference did not reach statistical significance. No compensatory increase in Nudt7 transcript levels was detected in the kidneys of the $\mathrm{Nudt} 19^{-/-}$mice in either the fed or fasted states (Fig. 2.2E). Combined, these data showed that Nudt19 was the major CoA diphosphohydrolase in the kidneys and that this enzyme contributed to the regulation of kidney CoA levels in vivo.

Mouse Nudt7 and other CoA diphosphohydrolases from S. cerevisiae and C. elegans localize to the peroxisomes $(17,22,37)$, which suggests that these enzymes might specifically regulate the peroxisomal pool of CoA $(38,39)$. Nudt19 contains a C-terminal peroxisome targeting signal type 1 (PTS1), Ala-HisLeu, and was originally identified as a peroxisomal enzyme by the proteomic analysis of kidney peroxisomes (16). Recently, proteomic studies conducted on isolated mitochondria have also led to the annotation of Nudt19 as a mitochondrial enzyme in the Mitocarta2.0 inventory (29). Given the common contamination of isolated peroxisomes and mitochondria with variable amounts of other organelles, we used immunofluorescence and confocal microscopy to determine the intracellular localization of Nudt19 in 
whole HEK 293 cells (Fig. 2.3). HEK 293 cells were transiently transfected with constructs expressing Nudt19 with N- or C-terminal GFP tags. Nuclei were stained with DAPI, mitochondria with Mitotracker ${ }^{\circledR}$ Orange CMTMRos, and peroxisomes were visualized with an antibody against the endogenous peroxisomal protein PMP70. When expressed with an N-terminal GFP tag that left the PTS1 exposed, Nudt19 co-localized with PMP70, as shown by the yellow pixels resulting from the overlap of the green and red contributions (Fig. 2.3A-C). Masking the PTS1 of Nudt19 with a C-terminal GFP tag prevented the peroxisomal localization (Fig. 2.3G-I) but did not direct the protein to the mitochondria (Fig. 2.3J-L), leading instead to a diffuse cytoplasmic localization. Overall, these results supported the conclusion that Nudt19 was a peroxisomal enzyme. As such, the $20 \%$ increase measured in the whole kidney homogenates from fed $N u d t 19^{-/-}$mice (Fig. 2.2D) could be an underestimation of the local accumulation of CoA in the kidney peroxisomes of these animals.

Nudt19, but not Nudt7, is Competitively Inhibited by Specific Bile Acids

Deletion of Nudt19 led to a significant increase in kidney CoA levels in fed but not fasted mice (Fig. 2.2D). This suggested that, in a wild type mouse, the activity of Nudt19 might be higher in the fed state compared to the fasted state. We analyzed Nudt19 mRNA and protein expression levels in the kidneys of mice fed ad libitum and mice fasted for up to 48h, but we did not detect any difference (Fig. 2.4A-C). We then focused our attention on metabolite regulation.

We screened a panel of 30 compounds, including a large number of substrates and products of peroxisomal metabolism, for their effect on the activity of recombinant Nudt19 (Table 2.1). None of the compounds tested significantly stimulated Nudt19 activity. Instead, we found that a select group of bile acids inhibited the enzyme by $>50 \%$. More specifically, chenodeoxycholic acid (CDCA) and its conjugated derivatives, taurochenodeoxycholic acid and glycochenodeoxycholic acid, decreased Nudt19 activity by 60-75\% with respect to vehicle control (Table 2.1). More hydrophobic bile acids, such as lithocholic acid and ursocholanic acid, were also potent inhibitors of Nudt19 and $\alpha$-muricholic acid, which derives from CDCA by the addition of a $6 \beta$-hydroxyl group, still retained significant potency against the enzyme. In 
contrast, cholic acid and its glycine and taurine conjugates exhibited decreased potency against Nudt19 compared to CDCA. Furthermore, other steroid compounds, such as progesterone, pregnenolone and corticosterone, had no significant effect on the activity of the enzyme, confirming the specificity of the inhibitory effect of CDCA and derived bile acids on Nudt19 (Table 2.1).

Interestingly, none of the compounds tested, including CDCA, significantly affected the activity of Nudt7 (Table 1). In addition, isothermal titration calorimetry (ITC) studies showed no detectable binding of CDCA to the liver enzyme, while the $\mathrm{K}_{\mathrm{d}}$ of the Nudt19-CDCA complex was determined to be $8.3 \mu \mathrm{M}$ $(\Delta \mathrm{G}=-6.9 \mathrm{kcal} / \mathrm{mol})$ (Fig. 2.5A and B). Further kinetic analysis of the mechanism of Nudt19 inhibition revealed that CDCA was a competitive inhibitor with respect to free CoA (Fig. 2.5C). Combined, these results identified CDCA as a specific competitive inhibitor of Nudt19 in vitro.

The CoA Boxes and the Nudix Signature Sequences of Nudt19 and Nudt7 are not Interchangeable

Bile acids are primarily synthesized and conjugated in the liver. While the potential relevance of Nudt19 inhibition by a select group of these compounds in vivo is currently not known, the lack of CDCA binding to Nudt7 and the competitive nature of the CDCA inhibition of Nudt19 strongly suggested that the CoA binding sites of the two enzymes might be different. Nudix hydrolases that degrade CoA contain a conserved motif, (L/M)(L/F)TXR(S/A)[3X](R/K)[3X]G[3X]FPGG, here referred to as the CoA box, which is proposed to play a role in CoA binding (24) (Fig. 2.6A). Based on the structures of both human NUDT7 and the $D$. radiodurans homolog, the CoA box comprises the three $\beta$ strands $\beta 2, \beta 3$ and $\beta 4$, while the Nudix signature sequence, $\mathrm{G}_{1}{ }^{\mathrm{N}}[5 \mathrm{X}] \mathrm{E}_{7}{ }^{\mathrm{N}}[7 \mathrm{X}] \mathrm{R}_{15}{ }^{\mathrm{N}} \mathrm{E}_{16}{ }^{\mathrm{N}} \mathrm{XXE}_{19}{ }^{\mathrm{N}} \mathrm{E}_{20}{ }^{\mathrm{N}} \mathrm{XG}_{22}{ }^{\mathrm{N}} \mathrm{U}$, here referred to as the Nudix box, forms a $\beta$-strand-loop/helix/loop structural motif (Fig. 2.6A) (21,40,41). In several validated CoA diphosphohydrolases, the Nudix box exhibits a variable number of ' $\mathrm{X}$ ' residues between $\mathrm{E}_{7}{ }^{\mathrm{N}}$ and $\mathrm{R}_{15}{ }^{\mathrm{N}}$. The most extreme case is represented by mouse and human Nudt19, which contain a 45-49 amino acid insertion (Fig. 2.6A). Given its position within the Nudix box, this insertion would occur between strand $\beta 4$ and helix $\alpha 2$, i.e. in the first loop of the Nudix $\beta$-strand-loop/helix/loop structural motif (Fig. 2.6A). 
We swapped the CoA box in Nudt7 and Nudt19, generating a chimeric Nudt7 with the Nudt19 CoA box, Nudt7-19-CB, and a chimeric Nudt19 with the Nudt7 CoA box, Nudt19-7-CB. We also substituted the Nudix box of Nudt7 with that of Nudt19 producing a Nudt7-19-NB chimera. All three chimeras were expressed and purified in amounts similar or higher compared to the wild type Nudt7 and Nudt19. Conversely, exchanging the Nudix box in Nudt19 with the shorter Nudt7 Nudix box produced a protein that could be expressed but not purified as a soluble enzyme. Interestingly, a similar result was obtained when we expressed a Nudt19( $\Delta 58-103)$ mutant lacking the unique insertion sequence in the Nudix box (data not shown), suggesting that this amino acid stretch could be important for the folding and/or stability of Nudt19. The three soluble chimeras were analyzed by analytical gel filtration to assess their folding. Based on their calculated molecular weight, about 70\% of Nudt19-7-CB and Nudt7-19-CB eluted as globular monomers, similar to wild type Nudt7 and Nudt19 (Fig. 2.6B). Smaller peaks, indicating the formation of multimers, were also detected in both the chimeras and the wild type enzymes (Fig. 2.6B). Despite evidence of protein folding, the specific activities of Nudt19-7-CB, Nudt7-19-CB and Nudt7-19NB were decreased 2-3 orders of magnitude compared to the respective wild type enzymes (Fig. 2.6C). In particular, the substitution of the CoA box of Nudt19 with that of Nudt7 caused $>5,000$-fold decrease in the $\mathrm{k}_{\text {cat }}$ and a 3-fold increase in the $\mathrm{K}_{\mathrm{M}}$ for free CoA compared to Nudt19 (Table 2.2). Similarly, swapping the Nudt7 CoA box with the CoA box of Nudt19 in Nudt7-19-CB led to a 500-fold decrease in $\mathrm{k}_{\text {cat }}$ and a 5-fold increase in $\mathrm{K}_{\mathrm{M}}$. Finally, exchanging the Nudix box in Nudt7-19-NB caused a 200-fold decrease in $\mathrm{k}_{\text {cat }}$ and a 3-fold increase in $\mathrm{K}_{\mathrm{M}}$ compared to Nudt7 (Table 2.2).

Wild type Nudt19 and Nudt7 readily degrade free CoA and lauroyl-CoA but exhibit different activities against acetyl-CoA and mBB-CoA, which are good substrates for Nudt7 but not Nudt19 (Fig. 2.1A and 1B). We next determined the effect of swapping the Nudix and CoA boxes on the substrate specificity of the three soluble chimeras Nudt19-7-CB, Nudt7-19-CB and Nudt7-19-NB (Fig. 2.6D). For each enzyme, the activity data with the different substrates were plotted relative to free CoA to allow for comparison across enzymes with dramatically different specific activities. All three proteins, including the Nudt19 chimera Nudt19-7-CB, degraded acetyl-CoA and mBB-CoA, with a significantly higher relative 
preference for the latter substrate compared to wild type Nudt7 and Nudt19. Combined with the dramatic reduction in $\mathrm{k}_{\text {cat }}$ and robust decrease in $\mathrm{K}_{\mathrm{M}}$ (Table 2.2), the altered substrate specificity of the chimeras suggested that swapping the Nudix and CoA boxes between Nudt19 and Nudt7 might have caused a substantial deformation of the respective active sites.

\section{Mutagenesis-guided Modeling of Nudt19}

The recently released crystal structure of human NUDT7 is the only currently available structure of a mammalian CoA diphosphohydrolase. This structure does not contain any divalent cation, essential to correctly orient the diphosphate bond of the substrate for hydrolysis, or CoA bound (23). To identify Nudt19 residues involved in CoA and possibly CDCA binding, we decided to build a model of the putative structure of Nudt19 in complex with $\mathrm{Mg}^{2+}$ and free $\mathrm{CoA}$ using a computational approach guided by mutational analysis.

Within the Nudix box, the carbonyl group of $\mathrm{G}_{1}{ }^{\mathrm{N}}$ and two of the three glutamates in the $\mathrm{E}_{16}{ }^{\mathrm{N}}, \mathrm{E}_{19}{ }^{\mathrm{N}}$ and $\mathrm{E}_{20}{ }^{\mathrm{N}}$ series, participate in the coordination of the essential divalent cation (24,40-45). $\mathrm{E}_{16}{ }^{\mathrm{N}}, \mathrm{E}_{19}{ }^{\mathrm{N}}$ and $\mathrm{E}_{20}{ }^{\mathrm{N}}$ correspond to E112, 115 and 116 in Nudt19, respectively (Fig. 2.6A and 2.7C). Mutation of these residues to alanine abolished the enzymatic activity of Nudt19 E112A and Nudt19 E116A (Table 2.2). The activity of Nudt19 E115A was detectable; however, the enzyme exhibited a 2000-fold decrease in $\mathrm{k}_{\text {cat }}$ and a 3-fold increase in $K_{M}$ (Table 2.2). Interestingly, the effect of this mutation on the $K_{M}$ suggested that this residue of the Nudix box contributed to CoA binding. We also mutated the equivalent Nudt7 residues $\mathrm{E}_{16}{ }^{92}$, $\mathrm{E}_{19}{ }^{95}$ and $\mathrm{E}_{20}{ }^{96}$. Similar to the Nudt19 mutants, Nudt7 E92A and Nudt7 E96A did not show any detectable activity. Mutation of Nudt7 E95 to alanine decreased the $\mathrm{k}_{\text {cat }}>10$-fold but, unlike the equivalent mutant in Nudt19, it had no significant effect on the $\mathrm{K}_{\mathrm{M}}$ for CoA. Overall, these results were consistent with mutagenesis studies conducted on other Nudix hydrolases $(42,46,47)$, and confirmed the importance of $\mathrm{E}_{16}{ }^{\mathrm{N}}, \mathrm{E}_{19}{ }^{\mathrm{N}}$ and $\mathrm{E}_{20} \mathrm{~N}$ for the activity of both Nudt19 and Nudt7. The crystal structure of the CoA diphosphohydrolase from $D$. radiodurans, CoAse, contains $\mathrm{Mg}^{2+}$ bound (24). Although unable to fit completely, docking of free CoA onto the 'closed' structure of the bacterial CoAse suggested the existence of a potential contact between the side chain of R55, the first arginine in the CoA box (Fig. 2.6A), and the 
diphosphate bond of CoA (24). Additionally, mutation of the equivalent residue to a glutamine in the $A$. thaliana homolog AtCoAse led to a dramatic decrease in activity (48). This arginine residue is highly conserved across CoA diphosphohydrolases, and corresponds to R34 in Nudt19 and R60 in Nudt7 (Fig. 2.6A). Mutation of these residues to a methionine, an amino acid with a comparable side chain but no charge, dramatically decreased the $\mathrm{k}_{\text {cat }}$ of the Nudt19 R34M and Nudt7 R60M mutants by 200 and 2000fold, respectively, and caused a 2-3 fold increase in the $\mathrm{K}_{\mathrm{M}}$. Combined, these data supported the importance of this residue for catalysis.

We used this information as a guide to evaluate computational models of Nudt19 generated by using the YASARA modeling software suite (32). Specifically, the final model that we selected exhibited 1) the correct positioning of $\mathrm{E}_{16}{ }^{112}, \mathrm{E}_{19}{ }^{155}$ and $\mathrm{E}_{20}{ }^{116}$ with respect to $\mathrm{Mg}^{2+}$, 2) the appropriate orientation of the diphosphate moiety of free CoA with respect to $\mathrm{Mg}^{2+}$ to allow for catalysis, and 3) interaction of R34 with the diphosphate group of the substrate CoA (Fig. 2.7B-C). In this model, the CoA and Nudix boxes of Nudt19 folded into the expected structural motifs with very good superposition with the CoA and Nudix boxes of the D. radiodurans CoAse and human NUDT7 (Fig. 2.7A). Within the $\beta$-strand-loop/helix/loop structural motif of the Nudix box, the 45 amino acid insertion in Nudt19 was modeled as an expansion in the loop connecting the strand $\beta 4$ to the helix $\alpha 2$ and was mostly unstructured.

\section{Identification of Additional Residues Involved in CoA Binding and Hydrolysis}

Based on the model, we selected and mutated a set of Nudt19 residues predicted to participate in the binding of CoA (Table 2.2). In particular, we mutated R39, F40, L41, which are part of the CoA box, and R189 to alanine (Fig. 2.6A and 2.7C). In the Nudt19 model, the side chains of F40 and L41 extended towards the free thiol end of the CoA molecule, while the side chain of R39 extended towards the 3'phosphate of the CoA adenine ring (Fig. 2.7C). Mutation of F40 and L41 to alanine caused a robust 3- to 4-fold increase in the $\mathrm{K}_{\mathrm{M}}$, confirming that these residues played a role in CoA binding. In addition, the Nudt19 L41A mutant also showed a 3-fold decrease in $\mathrm{k}_{\text {cat. }}$ Mutation of R39 to an alanine did not have a significant effect ( $<2$-fold) on the $\mathrm{K}_{\mathrm{M}}$; however, removal of the positive charge caused a 3-fold decrease 
in $\mathrm{k}_{\text {cat }}$, indicating that this residue was nevertheless important for CoA hydrolysis. R189 was located in a loop region outside the CoA box and stretched towards the adenosine moiety of CoA (Fig. 2.7C). The Nudt19 R189A mutant exhibited a 3-fold increase in $\mathrm{K}_{\mathrm{M}}$ and a 2-fold decrease in $\mathrm{k}_{\mathrm{cat}}$, confirming that R189 was important for both CoA binding and hydrolysis. We further investigated the role of this residue by mutating R189 to a methionine. Unlike alanine, the side chain of methionine was able to functionally replace R189, thus suggesting the existence of potential non-polar side chain interaction(s) between R189 and CoA (Table 2.2). Since Nudt19 R34M, F40A, L41A and R189A mutants exhibited a reduced affinity for CoA (Table 2.2), we tested the sensitivity of these mutants to inhibition by the competitive inhibitor CDCA (Fig. 2.5C). At a concentration of $250 \mu \mathrm{M}$ of free CoA, the $\mathrm{IC}_{50}$ of CDCA was determined to be $125 \mu \mathrm{M}$ against wild type Nudt19. Among the above mutants, Nudt19 R34M retained $~ 80 \%$ of activity in the presence of CDCA (Fig. 2.7D), suggesting that R34 might be involved in both CoA and CDCA binding. Although one of the variable ' $\mathrm{X}$ ' residues in the Nudix box, Nudt19 D53 is highly conserved among the CoA diphosphohydrolases (Fig. 2.6A). In the Nudt19 model, this residue showed no interaction with CoA, but we nevertheless mutated it to alanine to determine the effect of the mutation on $\mathrm{K}_{\mathrm{M}}$ and $\mathrm{k}_{\text {cat }}$. Consistent with the model, the Nudt19 D53A mutant exhibited kinetic parameters similar to the wild type enzyme, and we obtained similar results with the equivalent Nudt7 D79A mutant (Table 2.2). The sequence alignment of the CoA diphosphohydrolases also showed that it was common to find a positively charged residue in the same position as Nudt19 R39, a residue involved in catalysis but not CoA binding (Table 2.2). We mutated the equivalent residue in Nudt7, K65, to alanine. The Nudt7 K65A mutant exhibited a 3-fold increase in both $\mathrm{K}_{\mathrm{M}}$ and $\mathrm{k}_{\mathrm{cat}}$, showing a differential effect on both kinetic parameters with respect to Nudt19. Finally, since F40 in Nudt19 was involved in substrate binding, we mutated the equivalent Nudt7 R66 to both alanine and methionine. Nudt7 R66A showed a $>10$-fold increase in $\mathrm{K}_{\mathrm{M}}$, with only a minor effect on $\mathrm{k}_{\text {cat }}$. Conversely, the Nudt7 R66M mutant exhibited kinetic parameters similar to the wild type enzyme (Table 2.2). These results supported the conclusion that the side chain of R66 participated in 
CoA binding through hydrophobic interactions, and suggested that F40 in Nudt19 and R66 in Nudt7 might be functionally similar.

\section{Discussion}

To gain insight into the physiological role of Nudt19, a Nudix enzyme with CoA diphosphohydrolase activity, one the major goals of this study was to determine the ability of the enzyme to regulate CoA levels in vivo. The present work demonstrates that Nudt19 degrades CoA and contributes to the regulation of this cofactor in the kidneys. Indeed, Nudt $19^{-/-}$mice exhibited a significant $20 \%$ increase in total CoA levels in whole kidney homogenates obtained from fed mice (Fig. 2.2D). This result is consistent with the very narrow tissue distribution of the protein, which showed the highest expression levels in the kidneys, with brain and skeletal muscle containing barely detectable levels of Nudt19 (Fig. 2.1C).

The second goal of this study was to address conflicting reports on the peroxisomal and mitochondrial localization of Nudt19, as peroxisomes and mitochondria contain separate pools of CoA and are the sites of distinct CoA-dependent metabolic processes $(7,49)$. Using immunofluorescence and constructs expressing Nudt19 with either a C-terminal or an N-terminal GFP tag, we showed that the enzyme resides in the peroxisomes and not in the mitochondria (Fig. 2.3), thus suggesting a specific role for Nudt19 in peroxisomal metabolism. In the peroxisomes, free CoA and acyl-CoAs are imported through dedicated transporters $(50,51)$ and participate in several processes including the $\alpha$ - and $\beta$-oxidation of fatty acids that are not substrates for the mitochondrial oxidative machinery, the synthesis of ether phospholipids and, in the liver, the final steps in bile acid synthesis and conjugation. Additionally, since imported CoA species and other molecules $>400$ Da cannot freely exit the peroxisomes, the Nudix-mediated hydrolysis of CoA species to 3',5'-ADP and 4'-(acyl)phosphopantetheine likely prevents CoA buildup in this organelle $(38,39)$. Indeed, it has been proposed that the function of peroxisomal CoA-degrading enzymes, including Nudt7 and now Nudt19, is to regulate the peroxisomal CoA pool and, in turn, peroxisomal lipid metabolism (38). Consistent with this proposed role, Nudt19 activity was highest with free CoA, which is required in 
multiple peroxisomal pathways, and with succinyl-CoA and C8-C12 acyl-CoAs, which are the oxidation products of dicarboxylic and very long-chain fatty acids, respectively (Fig 2.1A). Nudt19 was previously reported to possess mRNA decapping activity in vitro (28). Although detectable, the hydrolytic activity of Nudt19 against capped RNA was minimal when compared to that of established mRNA decapping enzymes such as Dcp2 and Nudt16 (28). Additionally, mRNA decapping occurs in cytosolic foci (52). Thus, the peroxisomal localization of Nudt19, combined with its effect on CoA levels in vivo, argues against a role for this enzyme in mRNA decapping.

The peroxisomal localization of Nudt19 and fact that the enzyme could affect just one of the subcellular CoA pools could also explain the relatively small increase in total CoA levels observed in whole kidney homogenates obtained from $N u d t 19^{-/}$mice in the fed state. In the fasted state, the concentration of kidney CoA was higher than in the fed state and similar between wild type control and $\mathrm{Nudt}^{-9^{--}}$mice. These results suggest that the increase in kidney CoA levels that occurs upon fasting may be promoted more by activation of the CoA biosynthetic pathway than by a decrease in CoA degradation. The flux through the CoA biosynthetic pathway is controlled by the pantothenate kinase (PanK) isoforms, and PanK1, which is the isoform that drives the increase in CoA levels observed in the liver of fasted mice, is also the major PanK isoform in the kidneys (13,53). Upon refeeding, a net decrease in CoA levels requires not only inhibition of CoA synthesis, but also active degradation of the cofactor accumulated in the fasted state. This might explain why the effect of deleting Nudt19 on kidney CoA levels was mostly detected in fed mice. Interestingly, although the $N u d t 19^{-/}$mice exhibited higher kidney CoA levels in the fed state compared to control mice, this concentration of the cofactor was still significantly lower than in the fasted state, suggesting the existence of residual CoA-degrading activity in the absence of Nudt19. Nudt7 and Nudt19 are the only currently confirmed CoA-degrading enzymes and, in the kidneys, Nudt7 is a minor isoform (Fig. 2.1C, and compare 2.2E to 2.4A). Under our experimental conditions, the production of 3',5'ADP in kidney extracts from Nudt19-/ mice was undetectable (Fig. 2.2C), and we did not observe any compensatory upregulation of Nudt7 (Fig. 2.2E). In spite of this, an accurate estimate of the contribution of Nudt7, if any, to the regulation of kidney CoA levels, will require the generation of mice with a deletion 
in both Nudt7 and Nudt19. Alternatively, CoA might be hydrolyzed to products other than 3',5'-ADP and 4'-(acyl)phosphopantetheine by a novel class of CoA-degrading enzymes that may or may not reside in the peroxisomes.

The narrow and non-overlapping tissue distribution of Nudt19 and Nudt7 in kidneys and liver, respectively, is intriguing. Another major goal of this study was to investigate the distinctive biochemical and regulatory properties of these enzymes to gain insight into the physiological roles that Nudt19 and Nudt7 may play in their respective organs. Nudt19 (42 kDa) is significantly larger than Nudt7 (27 kDa), and contains unique sequence regions, the most notable of which is the 45 amino acid insertion in the Nudix box, which are not present in Nudt7 (Fig. 2.6A). Nudt7 expression in the liver decreases in the fasted state and increases in the fed state, showing an inverse correlated with the hepatic concentration of CoA $(9,26)$. Conversely, Nudt19 mRNA and protein levels in the kidneys did not change between the fed and fasted states, in spite of the fact that this enzyme contributes to the regulation of kidney CoA levels.

While searching for potential small molecule regulators, we identified CDCA as a competitive inhibitor of Nudt19 that did not bind to Nudt7, strongly suggesting that these two enzymes possess distinct CoA binding sites (Fig. 2.5). Interestingly, binding of CDCA to Nudt19 depended, at least in part, on R34 (R60 in Nudt7), a highly conserved residue involved in CoA binding and hydrolysis in both Nudt19 and Nudt7 (Fig. 2.6A, 2.7D and Table 2.2). Additional evidence in support of a different organization of the CoA binding sites between the two isoforms is provided by the different activities that Nudt19 and Nudt7 displayed against substrates such as acetyl-CoA and mBB-CoA and by the dramatic decrease in $\mathrm{k}_{\mathrm{cat}}$ observed upon exchange of the CoA and Nudix boxes between the two enzymes. Furthermore, the invariant $\mathrm{E}_{19}{ }^{\mathrm{N}}$ in the Nudix box was found to participate in CoA binding in Nudt19 but not Nudt7, while the conserved (R/K) residue in the CoA box (corresponding to K65 in Nudt7 and R39 in Nudt19) was found to be involved in CoA binding in Nudt7 but not Nudt19.

No structural information on CoA diphosphohydrolases with CoA bound is currently available and this represents a limitation to the accurate prediction of the mode of CoA binding through homology models. In spite of this, mutational analysis based on the Nudt19 model that we generated allowed us to 
identify residues that are important for CoA binding and hydrolysis (Table 2.2). These residues were found both within and outside the CoA box. The Nudt19 model also shows that the 45 amino acid insertion within the Nudix box may be mostly disordered. Within a protein, disordered regions can exert different functions, including providing display sites for post-translational modification and/or mediating protein-protein interactions (54). There are only a few known examples of peroxisomal proteins regulated through reversible post-translational modifications $(55,56)$, and the role of this potentially disordered sequence, if any, in the regulation Nudt19 activity remains to be elucidated. We also docked $\mathrm{Mg}^{2+}$ and free CoA to the NUDT7 crystal structure to determine whether we could find a pose that would agree with the mutational analysis on the closely related mouse Nudt7, and provide us with a model of CoA binding to NUDT7. As for Nudt19, we selected the most likely models using the position of $\mathrm{E}_{16}{ }^{92}, \mathrm{E}_{19}{ }^{95}, \mathrm{E}_{20}{ }^{96}, \mathrm{R} 60, \mathrm{CoA}$ and $\mathrm{Mg}^{2+}$. In these models, R66 (equivalent to R66 in mouse Nudt7) would form a salt bridge with the diphosphate moiety of CoA (data not shown). While the 10-fold increase in $K_{M}$ of the Nudt7 R66A mutant supports the importance of R66 for substrate binding, the lack of effect of the R66M mutation on the kinetic parameters of the enzyme indicates that the requirement for R66 is likely spatial and geometric and not about the charge (Table 2.2). Thus, in this case, the docking of CoA to the structure of NUDT7 does not seem to provide a close representation of how CoA binds to this enzyme. Crystallization of NUDT7 and possibly Nudt19 with a substrate bound will be required to yield further details of CoA binding.

Finally, bile acids are synthesized de novo exclusively in the liver, with the last steps in the synthesis and conjugation to glycine and taurine occurring in the peroxisomes (57). While it may be unlikely for Nudt19 to encounter high concentrations of CDCA in kidney peroxisomes, Nudt7 is presumably exposed to high concentrations of CDCA, cholic acid, and conjugated derivatives in liver peroxisomes. Thus, it is tempting to speculate that the ability of Nudt7 to remain active even at high micromolar concentrations of bile acids, is one possible reason for the selection of this enzyme as the major CoA diphosphohydrolase in the liver. As for Nudt19, further studies, including an in depth characterization of the $\mathrm{Nudt} 19^{-/-}$mice, will be required to determine what selective advantage(s) this isoform may confer to the kidneys. 


\section{$\underline{\text { References }}$}

1. Choudhary, C., Weinert, B. T., Nishida, Y., Verdin, E., and Mann, M. (2014) The growing landscape of lysine acetylation links metabolism and cell signalling. Nature reviews. Molecular cell biology 15, 536-550

2. Hirschey, M. D., and Zhao, Y. (2015) Metabolic Regulation by Lysine Malonylation, Succinylation, and Glutarylation. Molecular \& cellular proteomics : MCP 14, 2308-2315

3. Resh, M. D. (2016) Fatty acylation of proteins: The long and the short of it. Progress in lipid research 63, 120-131

4. Daniotti, J. L., Pedro, M. P., and Valdez Taubas, J. (2017) The role of S-acylation in protein trafficking. Traffic

5. Sabari, B. R., Zhang, D., Allis, C. D., and Zhao, Y. (2017) Metabolic regulation of gene expression through histone acylations. Nature reviews. Molecular cell biology 18, 90-101

6. Horie, S., Ishii, H., and Suga, T. (1981) Changes in peroxisomal fatty acid oxidation in the diabetic rat liver. Journal of biochemistry $\mathbf{9 0}, 1691-1696$

7. Van Broekhoven, A., Peeters, M. C., Debeer, L. J., and Mannaerts, G. P. (1981) Subcellular distribution of coenzyme A: evidence for a separate coenzyme A pool in peroxisomes. Biochemical and biophysical research communications 100, 305-312

8. Peng, Y., and Puglielli, L. (2016) N-lysine acetylation in the lumen of the endoplasmic reticulum: A way to regulate autophagy and maintain protein homeostasis in the secretory pathway. Autophagy 12, 1051-1052

9. Leonardi, R., Rock, C. O., and Jackowski, S. (2014) Pank1 deletion in leptin-deficient mice reduces hyperglycaemia and hyperinsulinaemia and modifies global metabolism without affecting insulin resistance. Diabetologia 57, 1466-1475

10. Corbin, D. R., Rehg, J. E., Shepherd, D. L., Stoilov, P., Percifield, R. J., Horner, L., Frase, S., Zhang, Y. M., Rock, C. O., Hollander, J. M., Jackowski, S., and Leonardi, R. (2017) Excess coenzyme A reduces skeletal muscle performance and strength in mice overexpressing human PANK2. Molecular genetics and metabolism 120, 350-362

11. Dusi, S., Valletta, L., Haack, T. B., Tsuchiya, Y., Venco, P., Pasqualato, S., Goffrini, P., Tigano, M., Demchenko, N., Wieland, T., Schwarzmayr, T., Strom, T. M., Invernizzi, F., Garavaglia, B., Gregory, A., Sanford, L., Hamada, J., Bettencourt, C., Houlden, H., Chiapparini, L., Zorzi, G., Kurian, M. A., Nardocci, N., Prokisch, H., Hayflick, S., Gout, I., and Tiranti, V. (2014) Exome sequence reveals mutations in CoA synthase as a cause of neurodegeneration with brain iron accumulation. American journal of human genetics 94, 11-22

12. Zhou, B., Westaway, S. K., Levinson, B., Johnson, M. A., Gitschier, J., and Hayflick, S. J. (2001) A novel pantothenate kinase gene (PANK2) is defective in Hallervorden-Spatz syndrome. Nature genetics 28, 345-349

13. Leonardi, R., Rehg, J. E., Rock, C. O., and Jackowski, S. (2010) Pantothenate kinase 1 is required to support the metabolic transition from the fed to the fasted state. PloS one 5, e11107

14. Jackowski, S., and Leonardi, R. (2014) Deregulated coenzyme A, loss of metabolic flexibility and diabetes. Biochemical Society transactions 42, 1118-1122

15. Zhang, Y. M., Chohnan, S., Virga, K. G., Stevens, R. D., Ilkayeva, O. R., Wenner, B. R., Bain, J. R., Newgard, C. B., Lee, R. E., Rock, C. O., and Jackowski, S. (2007) Chemical knockout of pantothenate kinase reveals the metabolic and genetic program responsible for hepatic coenzyme A homeostasis. Chemistry \& biology 14, 291-302

16. Ofman, R., Speijer, D., Leen, R., and Wanders, R. J. (2006) Proteomic analysis of mouse kidney peroxisomes: identification of RP2p as a peroxisomal nudix hydrolase with acyl-CoA diphosphatase activity. The Biochemical journal 393, 537-543

17. Gasmi, L., and McLennan, A. G. (2001) The mouse Nudt7 gene encodes a peroxisomal nudix hydrolase specific for coenzyme A and its derivatives. The Biochemical journal 357, 33-38 
18. Bessman, M. J., Frick, D. N., and O'Handley, S. F. (1996) The MutT proteins or "Nudix" hydrolases, a family of versatile, widely distributed, "housecleaning" enzymes. The Journal of biological chemistry 271, 25059-25062

19. Carreras-Puigvert, J., Zitnik, M., Jemth, A. S., Carter, M., Unterlass, J. E., Hallstrom, B., Loseva, O., Karem, Z., Calderon-Montano, J. M., Lindskog, C., Edqvist, P. H., Matuszewski, D. J., Ait Blal, H., Berntsson, R. P. A., Haggblad, M., Martens, U., Studham, M., Lundgren, B., Wahlby, C., Sonnhammer, E. L. L., Lundberg, E., Stenmark, P., Zupan, B., and Helleday, T. (2017) A comprehensive structural, biochemical and biological profiling of the human NUDIX hydrolase family. Nature communications 8, 1541

20. McLennan, A. G. (2006) The Nudix hydrolase superfamily. Cellular and molecular life sciences : CMLS 63, 123-143

21. de la Pena, A. H., Suarez, A., Duong-Ly, K. C., Schoeffield, A. J., Pizarro-Dupuy, M. A., Zarr, M., Pineiro, S. A., Amzel, L. M., and Gabelli, S. B. (2015) Structural and Enzymatic Characterization of a Nucleoside Diphosphate Sugar Hydrolase from Bdellovibrio bacteriovorus. PloS one 10, e0141716

22. Cartwright, J. L., Gasmi, L., Spiller, D. G., and McLennan, A. G. (2000) The Saccharomyces cerevisiae PCD1 gene encodes a peroxisomal nudix hydrolase active toward coenzyme A and its derivatives. The Journal of biological chemistry 275, 32925-32930

23. Crystal structure of Human Peroxisomal coenzyme A diphosphatase NUDT7. TO BE PUBLISHED

24. Kang, L. W., Gabelli, S. B., Bianchet, M. A., Xu, W. L., Bessman, M. J., and Amzel, L. M. (2003) Structure of a coenzyme A pyrophosphatase from Deinococcus radiodurans: a member of the Nudix family. Journal of bacteriology 185, 4110-4118

25. Shumar, S. A., Fagone, P., Alfonso-Pecchio, A., Gray, J. T., Rehg, J. E., Jackowski, S., and Leonardi, R. (2015) Induction of Neuron-Specific Degradation of Coenzyme A Models Pantothenate Kinase-Associated Neurodegeneration by Reducing Motor Coordination in Mice. PloS one 10, e0130013

26. Reilly, S. J., Tillander, V., Ofman, R., Alexson, S. E., and Hunt, M. C. (2008) The nudix hydrolase 7 is an Acyl-CoA diphosphatase involved in regulating peroxisomal coenzyme A homeostasis. Journal of biochemistry 144, 655-663

27. Rheaume, C., Barbour, K. W., Tseng-Crank, J., and Berger, F. G. (1989) Molecular genetics of androgen-inducible RP2 gene transcription in the mouse kidney. Molecular and cellular biology $\mathbf{9}$, 477-483

28. Song, M. G., Bail, S., and Kiledjian, M. (2013) Multiple Nudix family proteins possess mRNA decapping activity. Rna 19, 390-399

29. Calvo, S. E., Clauser, K. R., and Mootha, V. K. (2015) MitoCarta2.0: an updated inventory of mammalian mitochondrial proteins. Nucleic acids research

30. Shimada, K., and Mitamura, K. (1994) Derivatization of thiol-containing compounds. Journal of chromatography. B, Biomedical applications 659, 227-241

31. Garcia, M., Leonardi, R., Zhang, Y. M., Rehg, J. E., and Jackowski, S. (2012) Germline deletion of pantothenate kinases 1 and 2 reveals the key roles for CoA in postnatal metabolism. PloS one 7, e40871

32. Krieger, E., and Vriend, G. (2014) YASARA View - molecular graphics for all devices - from smartphones to workstations. Bioinformatics 30, 2981-2982

33. Thompson, J. D., Higgins, D. G., and Gibson, T. J. (1994) CLUSTAL W: improving the sensitivity of progressive multiple sequence alignment through sequence weighting, position-specific gap penalties and weight matrix choice. Nucleic acids research 22, 4673-4680

34. Robert, X., and Gouet, P. (2014) Deciphering key features in protein structures with the new ENDscript server. Nucleic acids research 42, W320-324

35. Adams, P. D., Afonine, P. V., Bunkoczi, G., Chen, V. B., Davis, I. W., Echols, N., Headd, J. J., Hung, L. W., Kapral, G. J., Grosse-Kunstleve, R. W., McCoy, A. J., Moriarty, N. W., Oeffner, R., Read, R. J., Richardson, D. C., Richardson, J. S., Terwilliger, T. C., and Zwart, P. H. (2010) 
PHENIX: a comprehensive Python-based system for macromolecular structure solution. Acta crystallographica. Section D, Biological crystallography 66, 213-221

36. Snider, L. D., King, D., and Lingrel, J. B. (1985) Androgen regulation of MAK mRNAs in mouse kidney. The Journal of biological chemistry 260, 9884-9893

37. AbdelRaheim, S. R., and McLennan, A. G. (2002) The Caenorhabditis elegans Y87G2A.14 Nudix hydrolase is a peroxisomal coenzyme A diphosphatase. BMC biochemistry 3, 5

38. Hunt, M. C., Tillander, V., and Alexson, S. E. (2014) Regulation of peroxisomal lipid metabolism: the role of acyl-CoA and coenzyme A metabolizing enzymes. Biochimie 98, 45-55

39. Antonenkov, V. D., and Hiltunen, J. K. (2006) Peroxisomal membrane permeability and solute transfer. Biochimica et biophysica acta 1763, 1697-1706

40. Gabelli, S. B., Bianchet, M. A., Bessman, M. J., and Amzel, L. M. (2001) The structure of ADPribose pyrophosphatase reveals the structural basis for the versatility of the Nudix family. Nature structural biology 8, 467-472

41. Gabelli, S. B., Bianchet, M. A., Xu, W., Dunn, C. A., Niu, Z. D., Amzel, L. M., and Bessman, M. J. (2007) Structure and function of the E. coli dihydroneopterin triphosphate pyrophosphatase: a Nudix enzyme involved in folate biosynthesis. Structure 15, 1014-1022

42. Boto, A. N., Xu, W., Jakoncic, J., Pannuri, A., Romeo, T., Bessman, M. J., Gabelli, S. B., and Amzel, L. M. (2011) Structural studies of the Nudix GDP-mannose hydrolase from E. coli reveals a new motif for mannose recognition. Proteins 79, 2455-2466

43. Swarbrick, J. D., Bashtannyk, T., Maksel, D., Zhang, X. R., Blackburn, G. M., Gayler, K. R., and Gooley, P. R. (2000) The three-dimensional structure of the Nudix enzyme diadenosine tetraphosphate hydrolase from Lupinus angustifolius L. Journal of molecular biology 302, 11651177

44. Lin, J., Abeygunawardana, C., Frick, D. N., Bessman, M. J., and Mildvan, A. S. (1996) The role of Glu 57 in the mechanism of the Escherichia coli MutT enzyme by mutagenesis and heteronuclear NMR. Biochemistry 35, 6715-6726

45. O'Handley, S. F., Thirawatananond, P., Kang, L. W., Cunningham, J. E., Leyva, J. A., Amzel, L. M., and Gabelli, S. B. (2016) Kinetic and mutational studies of the adenosine diphosphate ribose hydrolase from Mycobacterium tuberculosis. Journal of bioenergetics and biomembranes 48, 557567

46. Abdelghany, H. M., Bailey, S., Blackburn, G. M., Rafferty, J. B., and McLennan, A. G. (2003) Analysis of the catalytic and binding residues of the diadenosine tetraphosphate pyrophosphohydrolase from Caenorhabditis elegans by site-directed mutagenesis. The Journal of biological chemistry 278, 4435-4439

47. Iwai, T., Kuramitsu, S., and Masui, R. (2004) The Nudix hydrolase Ndx1 from Thermus thermophilus HB8 is a diadenosine hexaphosphate hydrolase with a novel activity. The Journal of biological chemistry 279, 21732-21739

48. Kupke, T., Caparros-Martin, J. A., Malquichagua Salazar, K. J., and Culianez-Macia, F. A. (2009) Biochemical and physiological characterization of Arabidopsis thaliana AtCoAse: a Nudix CoA hydrolyzing protein that improves plant development. Physiologia plantarum 135, 365-378

49. Horie, S., Isobe, M., and Suga, T. (1986) Changes in CoA pools in hepatic peroxisomes of the rat under various conditions. Journal of biochemistry 99, 1345-1352

50. Agrimi, G., Russo, A., Scarcia, P., and Palmieri, F. (2012) The human gene SLC25A17 encodes a peroxisomal transporter of coenzyme A, FAD and NAD+. The Biochemical journal 443, 241-247

51. Kemp, S., Theodoulou, F. L., and Wanders, R. J. (2011) Mammalian peroxisomal ABC transporters: from endogenous substrates to pathology and clinical significance. British journal of pharmacology 164, 1753-1766

52. Sheth, U., and Parker, R. (2003) Decapping and decay of messenger RNA occur in cytoplasmic processing bodies. Science 300, 805-808 
53. Dansie, L. E., Reeves, S., Miller, K., Zano, S. P., Frank, M., Pate, C., Wang, J., and Jackowski, S. (2014) Physiological roles of the pantothenate kinases. Biochemical Society transactions 42, 10331036

54. van der Lee, R., Buljan, M., Lang, B., Weatheritt, R. J., Daughdrill, G. W., Dunker, A. K., Fuxreiter, M., Gough, J., Gsponer, J., Jones, D. T., Kim, P. M., Kriwacki, R. W., Oldfield, C. J., Pappu, R. V., Tompa, P., Uversky, V. N., Wright, P. E., and Babu, M. M. (2014) Classification of intrinsically disordered regions and proteins. Chemical reviews 114, 6589-6631

55. Ortega-Galisteo, A. P., Rodriguez-Serrano, M., Pazmino, D. M., Gupta, D. K., Sandalio, L. M., and Romero-Puertas, M. C. (2012) S-Nitrosylated proteins in pea (Pisum sativum L.) leaf peroxisomes: changes under abiotic stress. Journal of experimental botany 63, 2089-2103

56. Knoblach, B., and Rachubinski, R. A. (2010) Phosphorylation-dependent activation of peroxisome proliferator protein PEX11 controls peroxisome abundance. The Journal of biological chemistry 285, 6670-6680

57. Ferdinandusse, S., and Houten, S. M. (2006) Peroxisomes and bile acid biosynthesis. Biochimica et biophysica acta 1763, 1427-1440 


\section{$\underline{\text { Table and figure legends }}$}

Table 2.1. Effect of selected metabolites on the activity of Nudt19 and Nudt7. Nudt19 and Nudt7 were assayed in the presence of $250 \mu \mathrm{M}$ of each compound, unless otherwise indicated, and free CoA as substrate. A '-' sign indicates a decrease in activity with respect to the vehicle control. A '+' sign indicates an increase in activity with respect to the vehicle control. Metabolites that decreased or increased the activity $\geq 50 \%$ are shaded in gray.

Table 2.2. Kinetic parameters of Nudt19 and Nudt7 mutants and chimeras. Experiments were conducted in duplicate as described in 'Experimental Procedures'. Kinetic constants were determined by non-linear regression analysis of the data and expressed \pm the standard error. ND indicates that the parameters were not determined due to undetectable enzymatic activity.

Figure 2.1. In vitro activity and tissue distribution of Nudt19 and Nudt7. Recombinant mouse Nudt19 (A) and Nudt7 (B) were incubated with $250 \mu \mathrm{M}$ of each CoA substrate, and the 3',5'-ADP produced was quantified as described in the "Experimental Procedures' section. Data are plotted as the mean (bars) of duplicate measurements from 2-3 independent experiments (circles) \pm standard deviation. (C) Immunoblot analysis to detect Nudt19 and Nudt7 proteins in mouse tissues. Tissue lysate proteins (150 $\mu \mathrm{g})$ were fractionated by SDS-PAGE, transferred to a PVDF membrane and blotted with the Nudt19 antibody. The same membrane was then stripped and blotted with antibodies against GAPDH (loading control) and Nudt7. Note that although the same amount of protein was loaded, different tissues contain a different amount of GAPDH. Abbreviations are: Panc., pancreas; WAT, white adipose tissue; BAT, brown adipose tissue; NS, non-specific band.

Figure 2.2. Effect of Nudt19 deletion on kidney CoA levels. Mouse genotyping. Multiplex PCR reactions were set up to detect the wild type and/or the knockout allele, as described in the 'Experimental Procedures' section. A PCR product of 185 bp indicated a wild type allele; a PCR product of 466 bp indicated a 
knockout allele. Heterozygous mice exhibited both bands. M, marker; WT, wild type; Hz, heterozygous; KO, knockout. (B) Western blot analysis of kidney homogenates $(150 \mu \mathrm{g})$ from wild type and Nudt19-/ mice using the Nudt19 antibody. GAPDH was used as a loading control. NS, nonspecific band. (C) Production of 3',5'-ADP in reaction mixtures containing free CoA and kidney extracts from wild type and Nudt $19^{-/-}$mice. Numbers in parenthesis represent the number of animals analyzed; data are reported as the mean \pm standard deviation. (D) Total CoA measured in the kidneys of wild type and Nudt19-/ mice fed ad libitum or fasted overnight (ON). (E) Nudt7 mRNA abundance in the kidneys of fed and fasted wild type and $N u d t 19^{-/-}$mice. Data in (D) and (E) are reported as the mean (bars) of measurements on individual mice (circles and squares) \pm standard deviation. ${ }^{* *}, p<0.01$ relative to wild type mice; \#, $p<0.05$, \#\#, $p<0.01$, $\# \#$ \# $p<0.001$ relative to the fed state.

Figure 2.3. Localization of Nudt19 to the peroxisomes. HEK 293 cells were transfected with expression plasmids encoding for GFP fused to (A-F) the N-terminus (Nudt19-N-GFP) or (G-L) the C-terminus of Nudt19 (Nudt19-C-GFP). Fixed cells were visualized using confocal microscopy. (B, C, H, I) The endogenous PMP70 protein was used as a marker for the peroxisomes, shown in red. (E, F, K, L) Mitochondria were visualized using Mitotracker Orange CMTMRos and shown in red. (A-L) Cell nuclei were stained with DAPI, in blue. (C, F, I, L) Insets in merged images show details at higher magnification. The results are representative of at least 2 independent experiments.

Figure 2.4. Nudt19 expression in the kidneys of fed and fasted mice. (A) Nudt19 transcript levels in the kidneys of fed, overnight fasted (ON), and 48h fasted mice. (B) Western blot analysis showing that Nudt19 protein levels in the kidneys did not change between fed, overnight fasted, and $48 \mathrm{~h}$ fasted mice. $\alpha$-Tubulin was used as a loading control. (C) Quantification of the western blot in (B). Data are reported as the mean (bars) of measurements on individual mice (circles, squares and diamonds) \pm standard deviation.

Figure 2.5. CDCA binds to Nudt19 but not to Nudt7. ITC binding curves of (A) Nudt19 and (B) Nudt7. The binding curves are representative of 2-3 independent experiments. (C) Graphical analysis of CDCA 
inhibition of Nudt19 using fixed concentrations of the inhibitor and variable concentrations of free CoA revealed that CDCA was competitive with free CoA. Data are reported as the mean of 2 independent experiments, in duplicate, \pm standard deviation.

Figure 2.6. Effect of exchanging the CoA and Nudix boxes of Nudt19 and Nudt7. (A) Alignment of the CoA and Nudix boxes of characterized CoA diphosphohydrolases from different organisms. Invariant residues are highlighted in red, conserved residues in yellow, and groups of conserved residues are boxed. The Nudix and the CoA boxes overlap at a glycine highlighted in black. Residues mutated in both Nudt19 and Nudt7 are marked with a blue star. Residues mutated just in Nudt19 are marked with a blue open circle. Secondary structure elements of the Deinococcus radiodurans CoAse (Dr1184, PDB ID: 1NQZ) and human NUDT7 (HsNUDT7, PDB ID: 5T3P) are shown on top of the alignment. The unique insertion sequence found in mouse and human Nudt19 would be inserted (blue triangle) in the loop region between the $\beta$ strand and the $\alpha$ helix of the Nudix box structural motif. Mm, Mus musculus; Hs, Homo sapiens; Dr, Deinococcus radiodurans; Sc, Saccharomyces cerevisiae; Ce, Caenorhabditis elegans. Genbank GI numbers for the aligned proteins are as follows: MmNudt7,165972342; HsNUDT77, 157785656; MmNudt19, 187954021; HsNUDT19, 157739940; Dr1184, 6458920; ScPcd1p, 6323180; CeNdx8, 68565843. (B) Calibration curve of the Superdex 75 10/300 GL column and elution profile of wild type Nudt19, Nudt7 and chimeras. The native molecular weights of the protein peaks, as estimated from the calibration curve, are reported for each enzyme. The calculated molecular weights of the recombinant Histagged proteins, based on the amino acid composition, are: wild type Nudt19, 42.6 kDa; Nudt19-7-CB, 42.6 kDa; wild type Nudt7, 29.2 kDa; Nudt7-19-NB, 34.0 kDa; Nudt7-19-CB, 29.1 kDa. (C) Specific activity of the recombinant Nudt19, Nudt7, and chimeric proteins. Specific activities \pm standard error were calculated from the linear regression of 5-point protein curves obtained for each enzyme from at least 2 independent experiments in duplicate. (D) Substrate specificity of Nudt19, Nudt7, and chimeric proteins. Activity with each CoA species is expressed as percentage relative to the activity with free CoA. Data are 
reported as the mean (bars) of duplicate measurements from 2-3 independent experiments (circles) \pm standard deviation. NB, Nudix box; CB, CoA box.

Figure 2.7. Homology model of Nudt19 with magnesium ion and free CoA bound. (A) Alignment of the CoA and Nudix box regions of the Nudt19 model (cyan) with the crystal structures of human NUDT7 (magenta, PDB ID: 5T3P) and D. radiourans CoAse (grey, PDB ID: 1NQZ). (B) The homology model for Nudt19 with $\mathrm{Mg}^{2+}$ bound (orange sphere) was obtained using the YASARA modeling software suite. Free CoA (sticks) was then docked using MOE and the resultant complex evaluated and selected as described in the text. Structural elements in the CoA and Nudix boxes are shown in green and cyan, respectively. The unique amino acid insertion in the Nudix box of Nudt19 is shown as a red dotted line in both (A) and (B). (C) Mode of CoA binding to Nudt19, with potentially interacting residues shown. (D) Activity of Nudt19 mutants incubated with or without the competitive inhibitor CDCA. Data are reported as the mean (bars) of duplicate measurements from 2-3 independent experiments (circles) \pm standard deviation. 
Table 2.1. Effect of selected metabolites on the activity of Nudt19 and Nudt7.

\begin{tabular}{|c|c|c|}
\hline \multirow[t]{2}{*}{ Metabolite } & \multicolumn{2}{|c|}{$\begin{array}{c}\text { \% Activity relative to } \\
\text { vehicle }\end{array}$} \\
\hline & Nudt19 & Nudt7 \\
\hline Hydrogen peroxide $(10 \mu \mathrm{M})$ & +16 & -11 \\
\hline Hydrogen peroxide (100 $\mu \mathrm{M})$ & +3 & +5 \\
\hline ATP & +6 & +2 \\
\hline $\mathrm{NAD}^{+}$ & -5 & +1 \\
\hline NADH & -13 & +20 \\
\hline $\mathrm{NADP}^{+}$ & -16 & +4 \\
\hline NADPH & -5 & +7 \\
\hline NMN & -8 & -1 \\
\hline Pyruvate & +13 & -17 \\
\hline$\alpha$-Ketoglutarate & 0 & -8 \\
\hline Isocitrate & -9 & -5 \\
\hline Sarcosine & +8 & +4 \\
\hline Carnitine & -3 & -1 \\
\hline Glycine & -4 & +16 \\
\hline Taurine & +5 & +24 \\
\hline Lauric acid & -16 & -4 \\
\hline Palmitic acid (50 $\mu \mathrm{M})$ & -2 & -32 \\
\hline Palmitoylcarnitine (50 $\mu \mathrm{M})$ & +23 & +11 \\
\hline Cholic acid & -24 & +8 \\
\hline Glycocholic acid & -30 & +10 \\
\hline Taurocholic acid & -17 & +3 \\
\hline Chenodeoxycholic acid & -62 & +1 \\
\hline Glycochenodeoxycholic acid & -67 & +3 \\
\hline Taurochenodeoxycholic acid & -75 & +11 \\
\hline$\alpha$-Muricholic acid & -50 & -8 \\
\hline Lithocholic acid (50 $\mu \mathrm{M})$ & -83 & -15 \\
\hline Ursocholanic acid (50 $\mu \mathrm{M})$ & -70 & +12 \\
\hline Corticosterone & -26 & -7 \\
\hline Progesterone & -37 & 0 \\
\hline Pregnenolone & -15 & +14 \\
\hline
\end{tabular}


Table 2.2. Kinetic parameters of Nudt19 and Nudt7 mutants and chimeras.

\begin{tabular}{c|cc}
\hline Mutation & $\mathbf{K}_{\mathbf{M}}(\boldsymbol{\mu M})$ & $\mathbf{k}_{\text {cat }}(\mathbf{1} / \mathbf{m i n})$ \\
\hline \multicolumn{4}{c}{ Nudt19 } \\
Nudt19-7-CB & $300 \pm 40$ & $284 \pm 12$ \\
E112A & ND & ND \\
E115A & $1019 \pm 226$ & $0.16 \pm 0.02$ \\
E116A & ND & ND \\
R34M & $680 \pm 186$ & $0.12 \pm 0.01$ \\
R39A & $511 \pm 64$ & $91 \pm 4$ \\
F40A & $1053 \pm 153$ & $210 \pm 12$ \\
L41A & $1250 \pm 125$ & $101 \pm 4$ \\
D53A & $434 \pm 59$ & $323 \pm 16$ \\
R189A & $1193 \pm 184$ & $118 \pm 11$ \\
R189M & $455 \pm 80$ & $177 \pm 10$ \\
\hline \multicolumn{4}{c}{ Nudt7 } \\
WT & $283 \pm 94$ & $125 \pm 12$ \\
Nudt7-19-CB & $1362 \pm 328$ & $0.28 \pm 0.03$ \\
Nudt7-19-NB & $836 \pm 173$ & $0.65 \pm 0.04$ \\
E92A & ND & ND \\
E95A & $444 \pm 87$ & $10 \pm 0.7$ \\
E96A & ND & ND \\
R60M & $1010 \pm 155$ & $0.55 \pm 0.03$ \\
K65A & $958 \pm 277$ & $463 \pm 56$ \\
R66A & $3069 \pm 407$ & $220 \pm 10$ \\
R66M & $496 \pm 79$ & $167 \pm 7$ \\
D79A & $334 \pm 29$ & $159 \pm 5$ \\
\hline
\end{tabular}



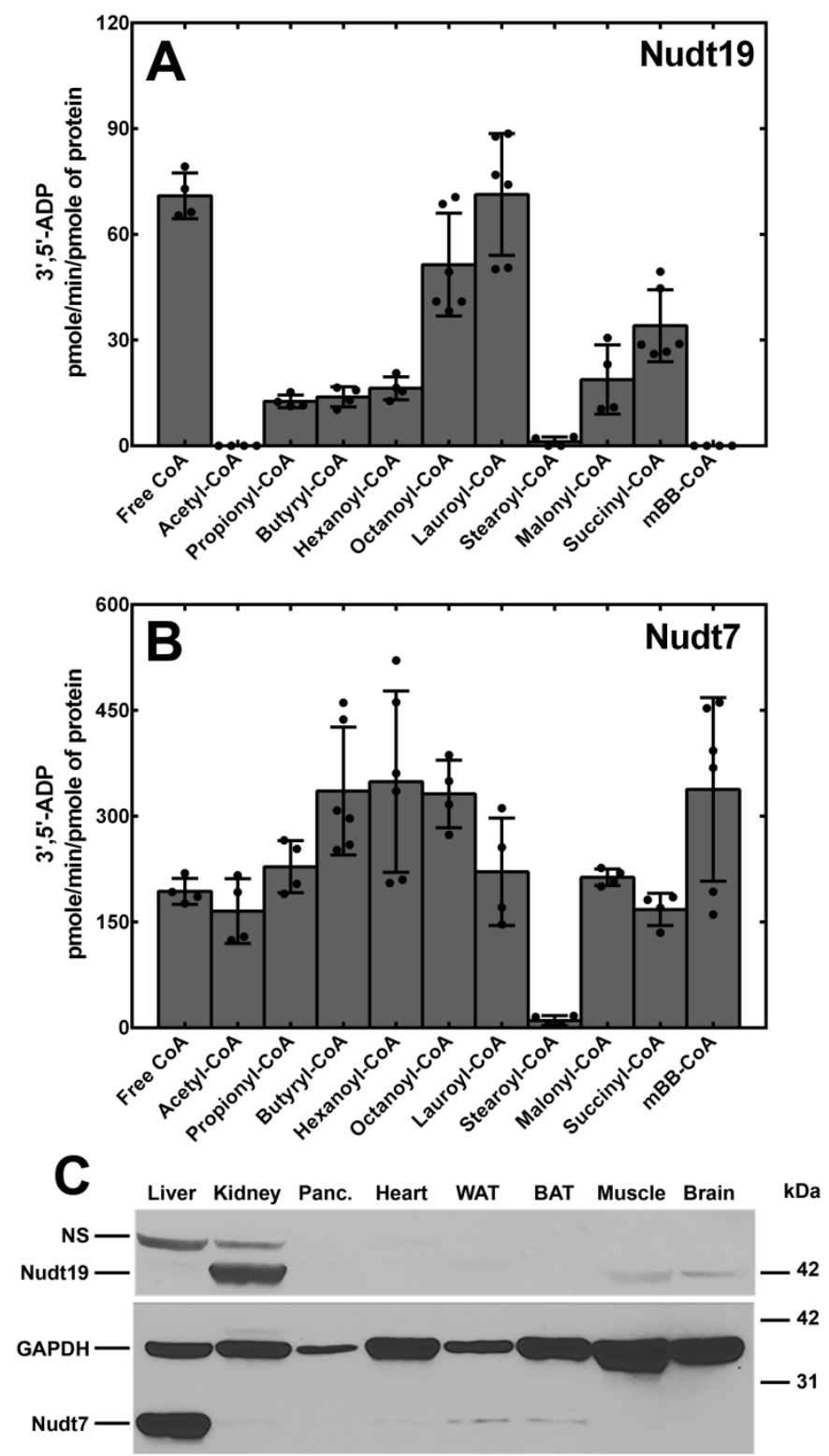

Figure 2.1. In vitro activity and tissue distribution of Nudt19 and Nudt7. 

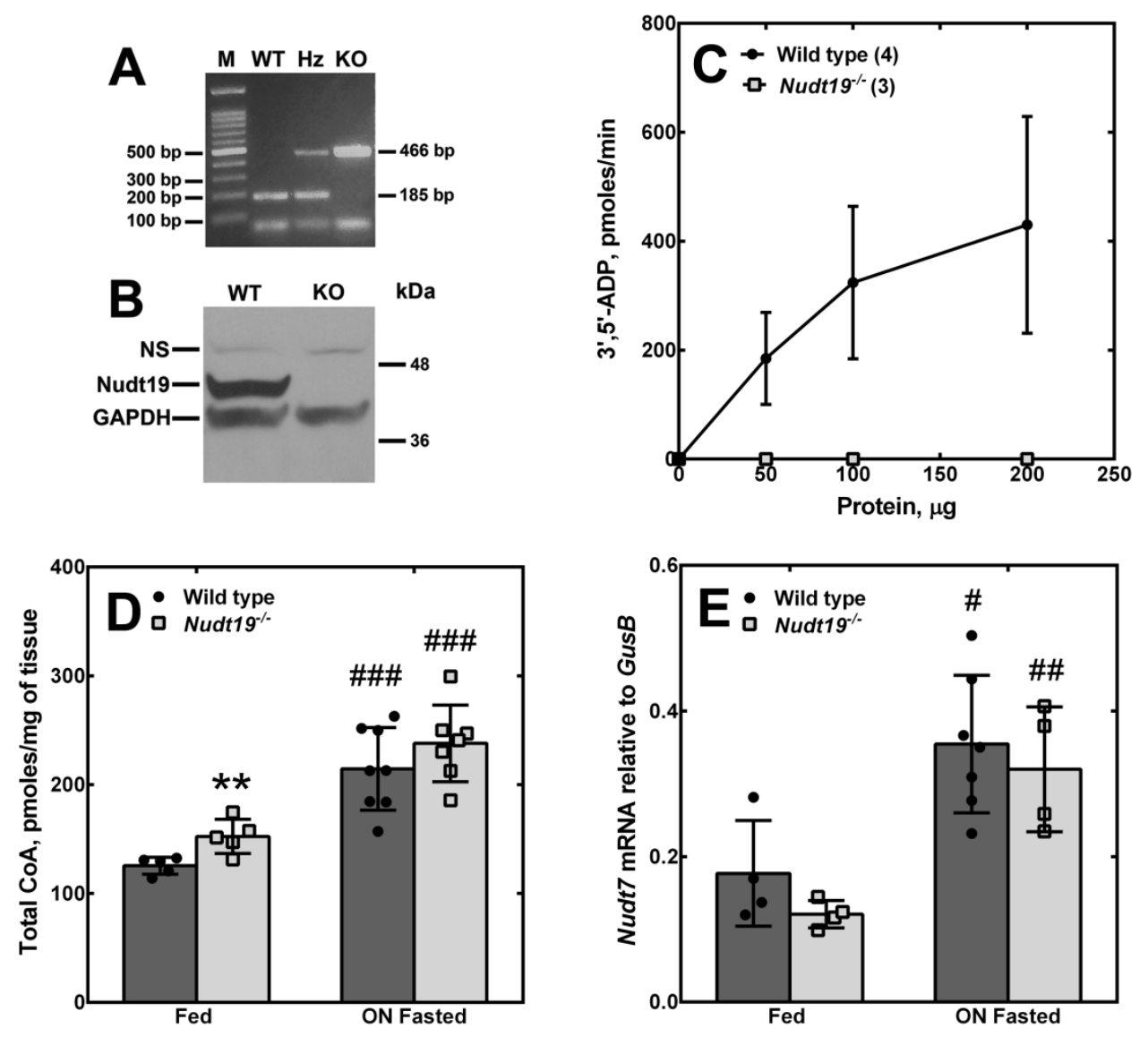

Figure 2.2. Effect of Nudt19 deletion on kidney CoA levels. 


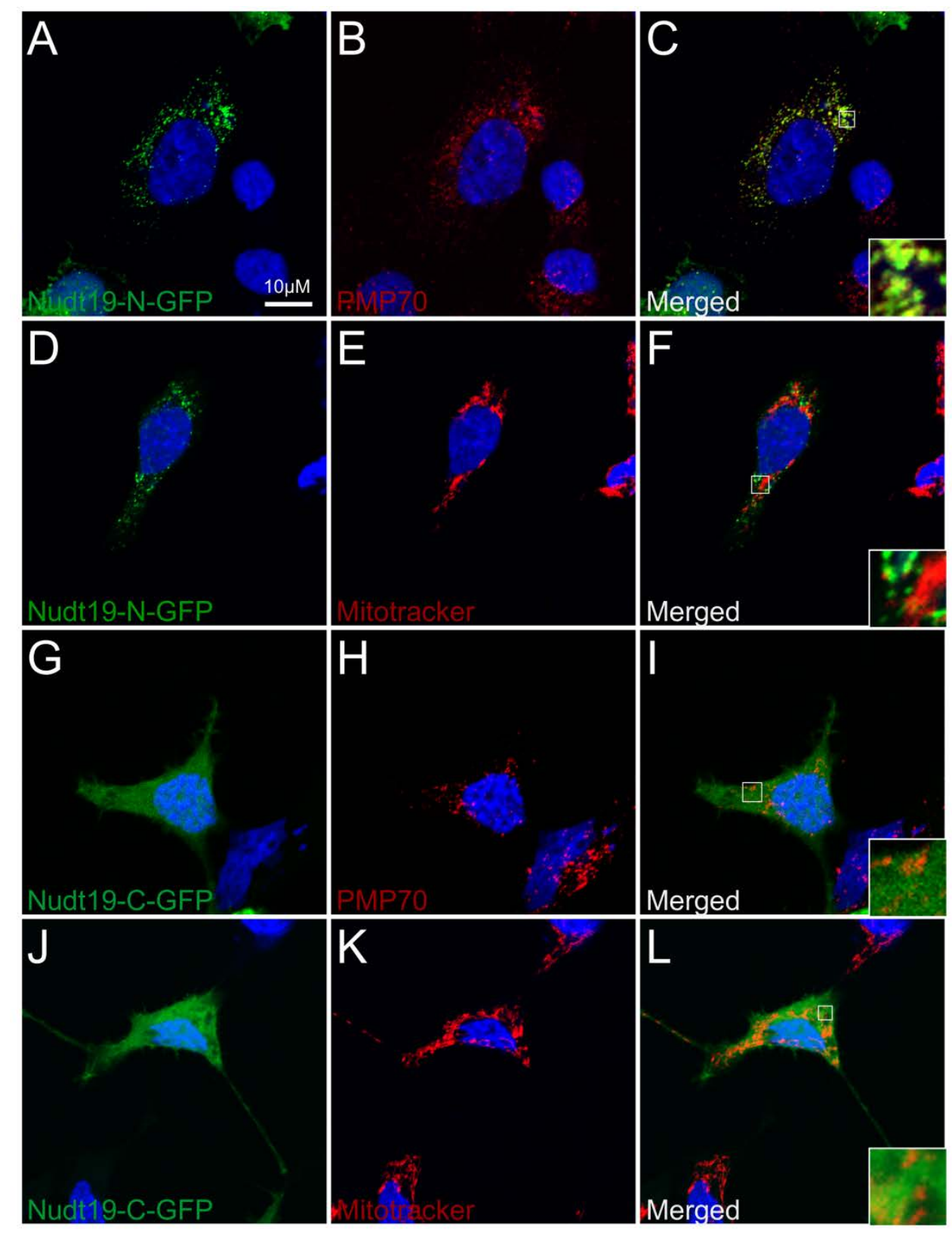

Figure 2.3. Localization of Nudt19 to the peroxisomes. 

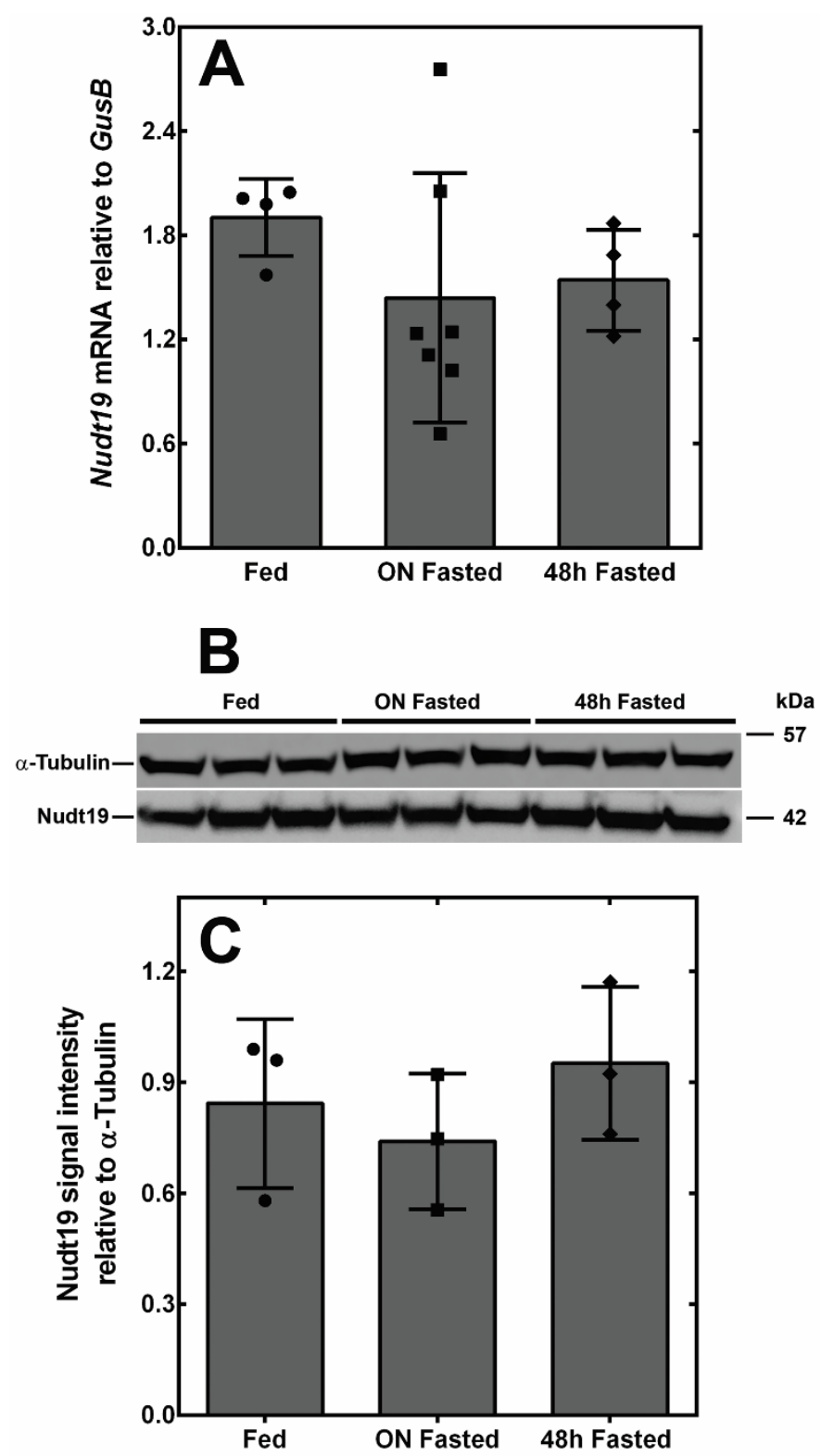

Figure 2.4. Nudt19 expression in the kidneys of fed and fasted mice. 

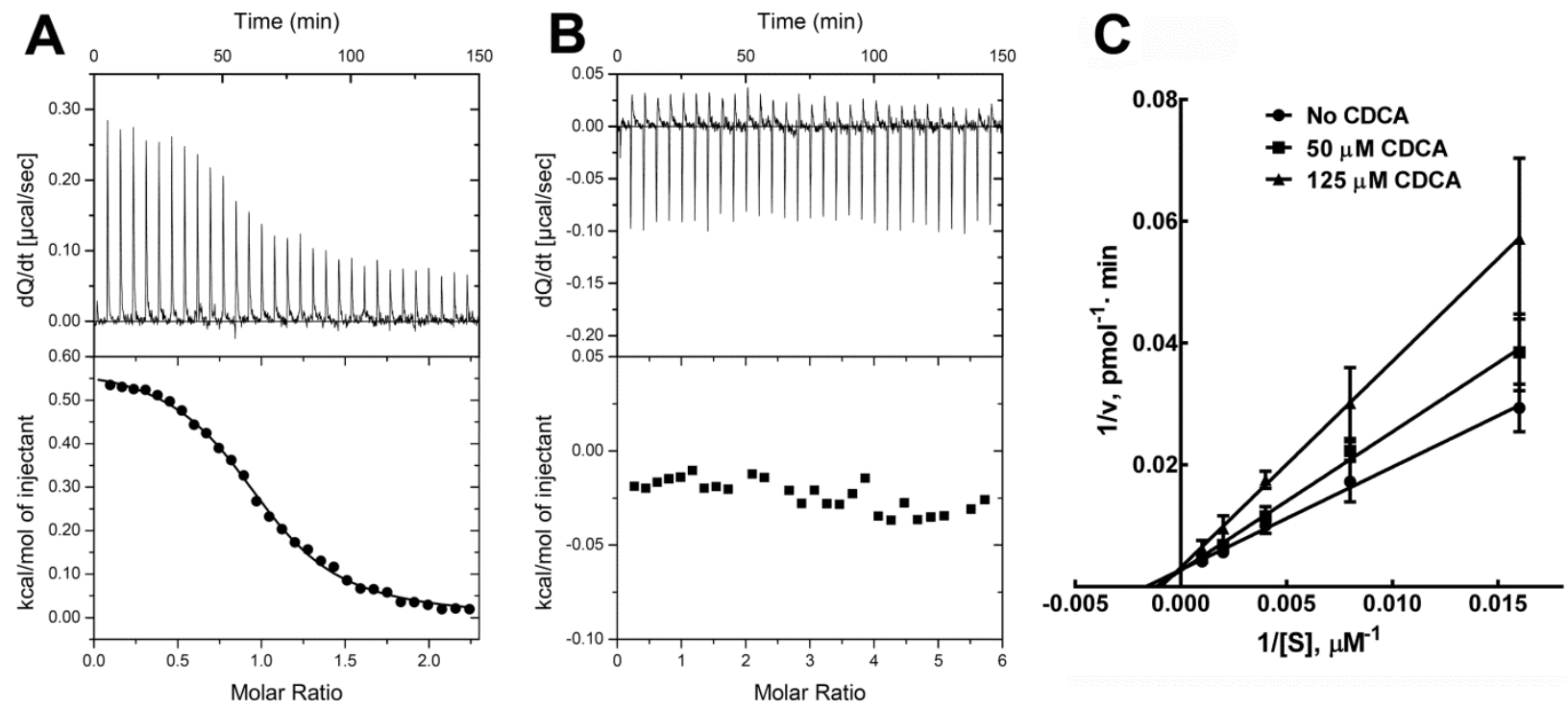

Figure 2.5. CDCA binds to Nudt19 but not to Nudt7. 


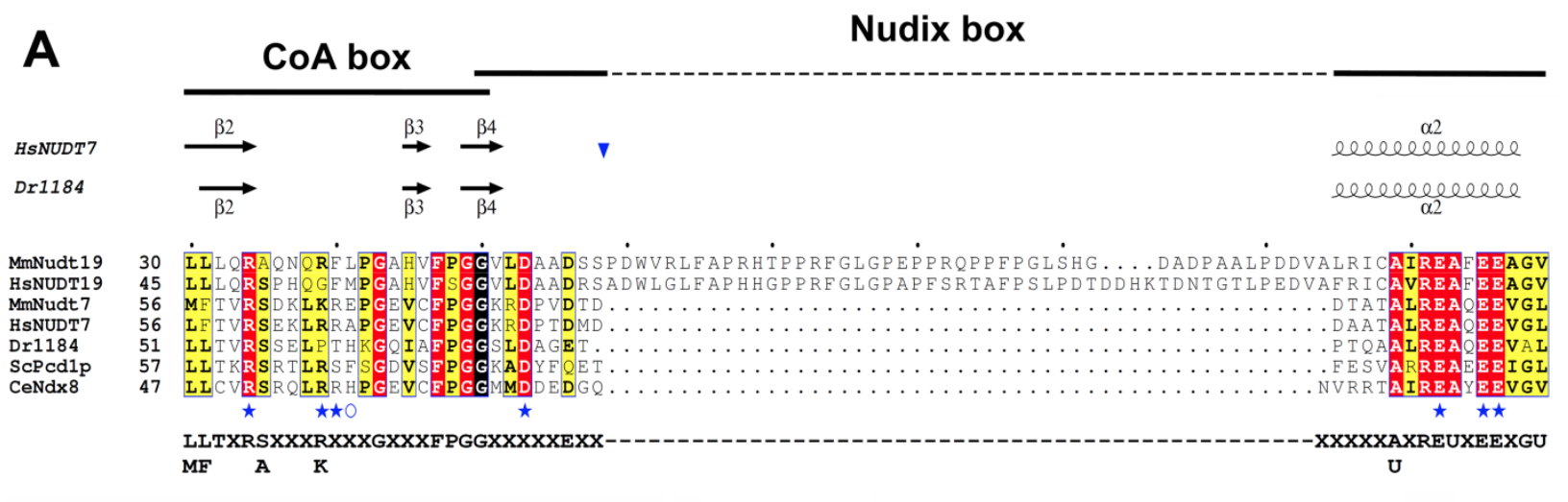

B

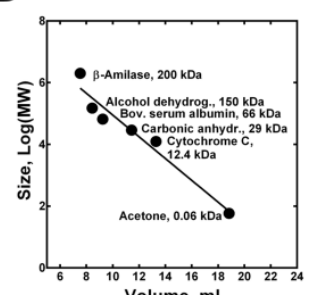

Volume, $\mathrm{ml}$
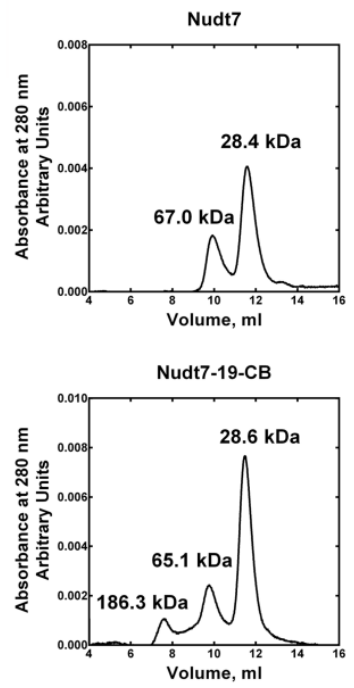
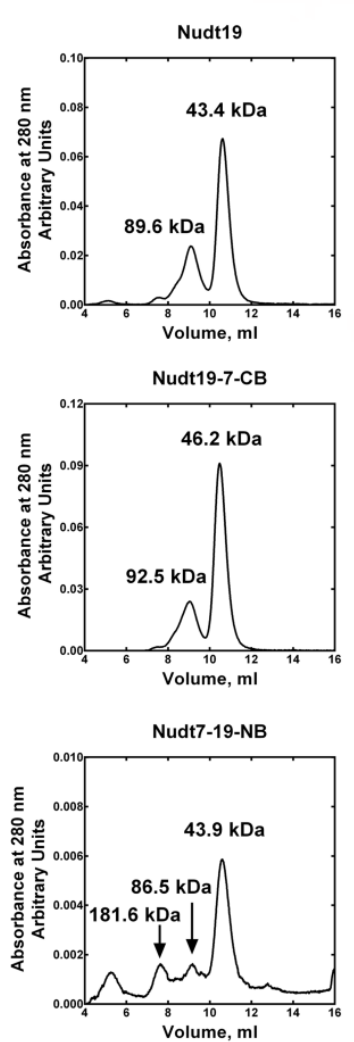

C

Specific activity with free CoA as substrate, $\mathrm{pmole} / \mathrm{min} / \mathrm{pmole}$ of protein

Nudt $19=89 \pm 6$

Nudt7 $=79 \pm 6$

Nudt19-7-CB $=0.014 \pm 0.001$

Nudt7-19-CB $=0.037 \pm 0.003$

Nudt7-19-NB $=0.139 \pm 0.025$

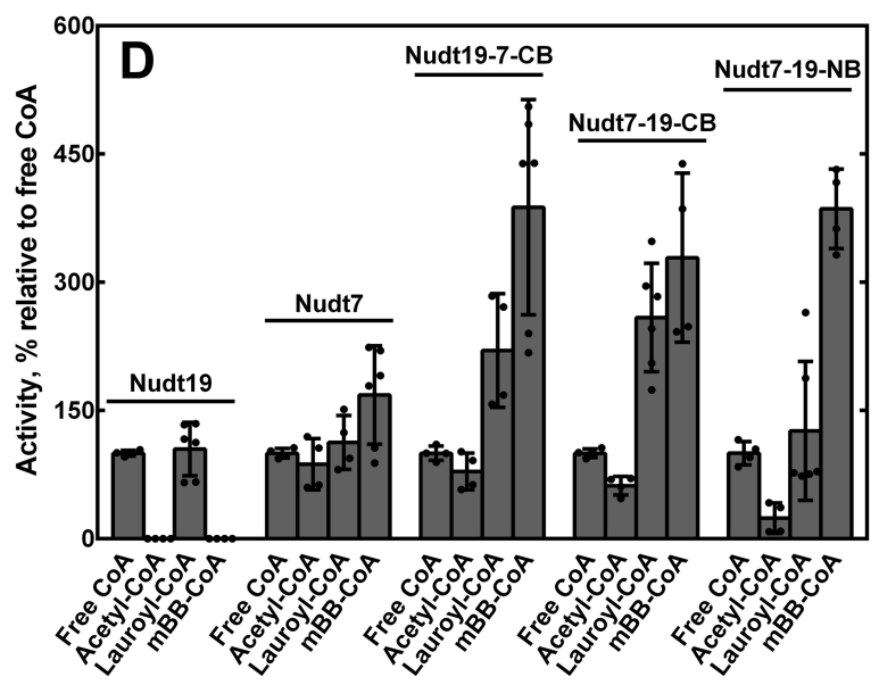

Figure 2.6. Effect of exchanging the CoA and Nudix boxes of Nudt19 and Nudt7. 

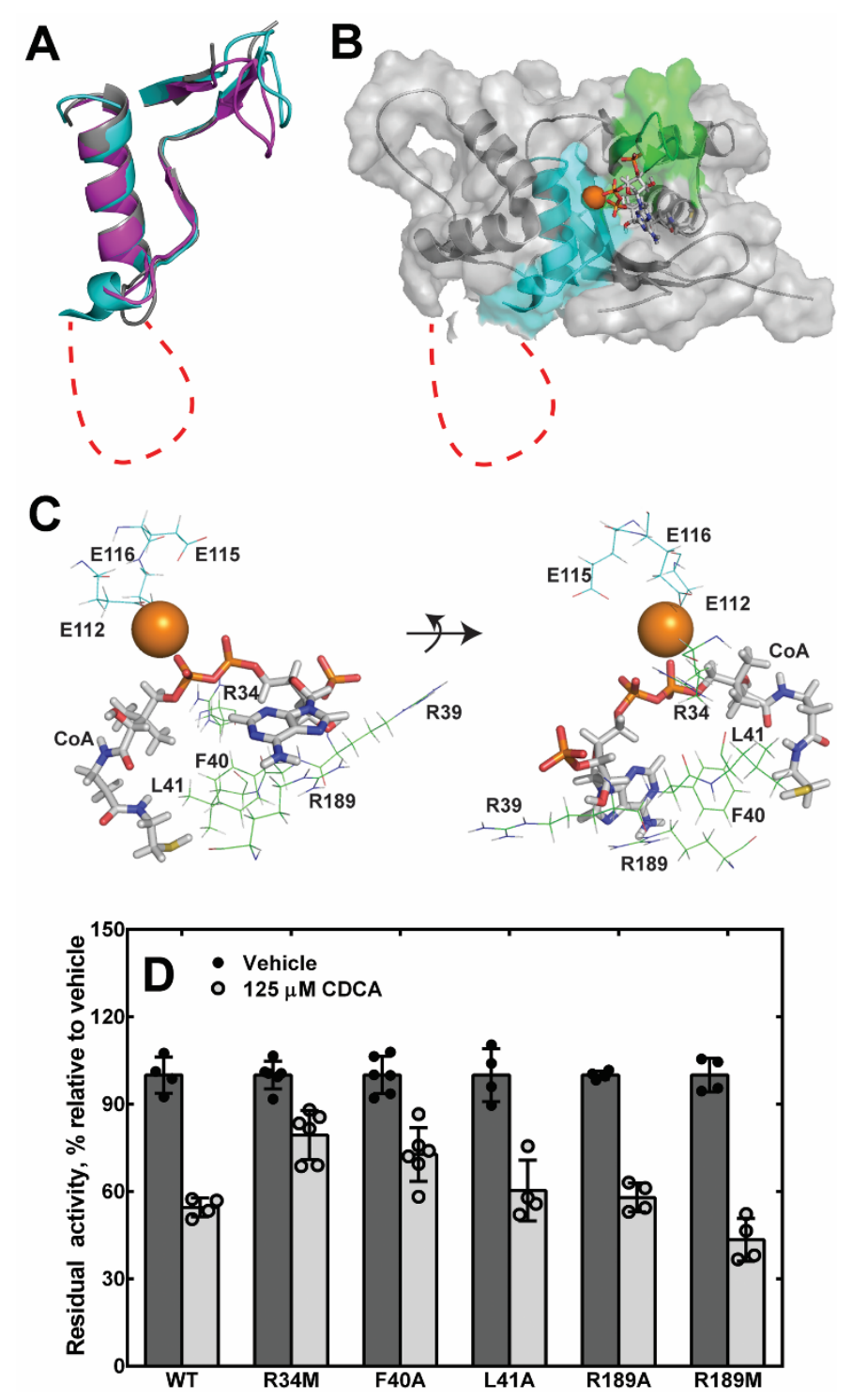

Figure 2.7. Homology model of Nudt19 with magnesium ion and free CoA bound. 
Chapter III: Identification of the in vivo CoA species and subcellular CoA pool(s) regulated by

\section{Nudt7}

Shumar, S. A., Kerr, E. W., Fagone, P., Infante, A. M., \& Leonardi, R. (2019). Overexpression of the CoA-degrading enzyme Nudt7 decreases liver bile acid levels and peroxisomal fatty acid oxidation. Journal of lipid research, jlr-M092676.

Author Contributions

$S A S, R L$, and EWK wrote the manuscript; SAS and RL designed the study; SAS conducted animal studies,CoA analysis, immunoblotting, lipid analysis, $\beta$ oxidation and enzymatic assays; EWK conducted the immunofluorescence studies; AMF analyzed RNAseq data; PF generated AAV. All authors analyzed the results and approved the final version of the manuscript.

This research was originally published in the Journal of Lipid Research. Shumar, S. A., Kerr, E. W., Fagone, P., Infante, A. M., \& Leonardi, R. Overexpression of the CoA-degrading enzyme Nudt7 decreases liver bile acid levels and peroxisomal fatty acid oxidation. J. Lipid Res. 2019; jlr-M092676. (c) The American Society for Biochemistry and Molecular Biology 


\begin{abstract}
$\underline{\text { Abstract }}$
Lipid metabolism requires coenzyme A (CoA), an essential cofactor found in multiple subcellular compartments including the peroxisomes. In the liver, CoA levels are dynamically adjusted between the fed and fasted states. Elevated CoA levels in the fasted state are driven by increased synthesis; however, this also correlates with decreased expression of Nudt7, the major CoA-degrading enzyme in the liver. Nudt7 resides in the peroxisomes and we overexpressed this enzyme in mouse livers to determine its effect on the size and composition of the hepatic CoA pool in the fed and fasted states. Nudt7 overexpression did not change total CoA levels but decreased the concentration of short-chain acyl-CoAs and choloyl-CoA in fasted livers, when endogenous Nudt7 activity was lowest. The effect on these acyl-CoAs correlated with a significant decrease in the hepatic bile acid content and in the rate of peroxisomal fatty acid oxidation, as estimated by targeted and untargeted metabolomics, combined with the measurement of fatty acid oxidation in intact hepatocytes. Identification of the CoA species and metabolic pathways affected the overexpression on Nudt7 in vivo supports the conclusion that the nutritionally-driven modulation of Nudt7 activity could contribute to the regulation of the peroxisomal CoA pool and peroxisomal lipid metabolism.
\end{abstract}




\section{Introduction}

The liver is a metabolically flexible organ that plays a key role in the maintenance of whole-body lipid and glucose homeostasis (1-3). Several pathways, including gluconeogenesis, ketogenesis, and bile acid synthesis occur primarily in this organ and rely on the availability of the essential cofactor coenzyme A (CoA). CoA is the major acyl group carrier in mammalian cells. Formation of acyl-CoAs allows for the delivery of activated substrates for a multitude of reactions and the modulation of metabolic pathways through allosteric regulation or posttranslational modification of histones and key metabolic enzymes (49). Cells contain limited and regulated amounts of CoA that are sequestered in multiple subcellular compartments, including the cytosol, peroxisomes, mitochondria, and endoplasmic reticulum (10-13). Within each subcellular compartment, free CoA and acyl-CoAs support specific pathways and reactions. These include ketogenesis, the TCA cycle and fatty acid $\beta$-oxidation in the mitochondria, fatty acid synthesis in the cytosol, autophagy in the endoplasmic reticulum, and the last steps in the bile acid biosynthetic pathway and fatty acid $\alpha$ - and $\beta$-oxidation in the peroxisomes $(3,12,14)$.

In the liver, CoA levels change in response to the nutritional state to support the switch between glucose and fatty acid oxidation $(11,15,16)$. This dynamic modulation occurs through a balance between CoA synthesis and degradation, which leads to a net increase in the concentration of hepatic CoA in the fasted state and to a net decrease in the fed state (16-18). CoA synthesis requires 5 enzymatic steps and is regulated through feedback inhibition of the first and rate-limiting enzyme of the pathway, pantothenate kinase, by free CoA and acyl-CoAs (19-21). The only currently known CoA-degrading enzymes are Nudt7 and Nudt19 $(22,23)$. Both enzymes are members of the Nudix hydrolase superfamily, specifically hydrolyze CoA species to produce 3',5'-ADP and (acyl-)phosphopantetheine, and reside in the peroxisomes, organelles that play a key role in lipid metabolism (18,22-26). Nudt19 is the major isoform in the kidneys, where it contributes to the regulation of total CoA levels in these organs (18). Nudt7 is the major CoA-degrading enzyme in the liver. Recombinant Nudt7 hydrolyzes a broad range of CoA substrates, including free CoA, short- and medium-chain acyl-CoAs, and the CoA thioesters of bile acids 
$(17,18)$. Unlike Nudt19, Nudt7 is not inhibited by bile acids and its expression varies with fasting and feeding. In particular, Nudt7 levels decrease with fasting, correlating with the increase in CoA observed in fasted livers. While these findings have established that Nudt7 can degrade CoA in vitro, it has yet to be determined whether Nudt7 is actively involved in regulating hepatic CoA levels in vivo. Indeed, the peroxisomal CoA pool is estimated to represent only $4-10 \%$ of total liver CoA $(10,11)$, and it is currently unclear to what extent the activity of Nudt7 in liver peroxisomes could affect total CoA levels in the fed and fasted states. Furthermore, the localization, substrate specificity, and nutritional regulation of Nudt7 expression suggest that this enzyme may be involved in the regulation of peroxisomal pathways (27), but direct evidence is lacking.

To gain insight into the physiological role of Nudt7 and its ability to regulate liver CoA levels in vivo, we overexpressed the enzyme in the liver of C57BL/6J mice. Overexpression of Nudt7 in the fasted state, when endogenous Nudt7 activity was lowest, did not prevent the increase in total hepatic CoA levels that occurs with fasting, but it changed the acyl-CoA composition of the liver by significantly decreasing the concentration of short-chain acyl-CoAs and choloyl-CoA, a peroxisomal intermediate in bile acid synthesis. Untargeted and targeted metabolomics analysis revealed that fasted livers overexpressing Nudt7 contained significantly reduced levels of primary and secondary bile acids, in addition to an accumulation of medium- and long-chain acylcarnitines, which correlated with a lower rate of peroxisomal fatty acid oxidation. Our data show that overexpression of Nudt7 specifically interfered with the output of peroxisomal fatty acid oxidation and the bile acid biosynthetic pathway in the fasted state. Thus, changes in Nudt7 activity between the fed and fasted states could act to regulate the peroxisomal CoA pool and, in turn, peroxisomal lipid metabolism. 


\section{Materials and methods}

Reagents were purchased from the following suppliers: HRP-conjugated GFP antibody, Alexa Fluor 647- and HRP-conjugated goat anti-rabbit IgG from Thermo Fisher Scientific; the antibodies against glyceraldehyde-3-phosphate dehydrogenase (GAPDH) and the FLAG epitope (DYKDDDK) from Cell Signaling; Waymouth MB 752/1 medium, medium 199 (M199) and the antibody against ATP-binding cassette, sub-family D, member 3 (PMP70) from Sigma-Aldrich. The Nudt7 antibody was generated as previously described (15). The fluorescent CoA derivative of monobromobimane (mBB), mBB-CoA, was synthesized as previously described (18). All other reagents were of analytical grade or better and were purchased from Sigma-Aldrich or Fisher Scientific, unless otherwise stated.

Adeno-associated virus production

Plasmid pscAAV-LP1-EGFP, carrying the enhanced green fluorescent protein coding sequence (henceforth referred to as GFP), was developed by John T. Gray and provided by St. Jude Children's Research Hospital, Memphis, TN. Plasmid pscAAV-LP1-mNudt7, obtained by replacing the coding sequence of GFP with the sequence of mouse Nudt7 containing a FLAG tag at the N-terminus, was a kind gift of Suzanne Jackowski, St. Jude Children's Research Hospital, Memphis, TN. To produce adenoassociated virus (AAV), HEK 293T cells were transiently transfected with a 1:1:2.5 (mass ratio) mixture of plasmids pHGTI-adeno1 (developed by John T. Gray and provided by Harvard College, Cambridge, MA), pLTAAVhelp2-8 (developed by John T. Gray and provided by St. Jude Children's Research Hospital, Memphis, TN) and either pscAAV-LP1-mNudt7 or pscAAV-LP1-EGFP using polyethylenimine. Transfected cells were harvested $72 \mathrm{~h}$ post transfection and lysed in $50 \mathrm{mM}$ Tris-HCl, $50 \mathrm{mM} \mathrm{NaCl,} 1 \mathrm{mM}$ $\mathrm{MgCl}_{2}$, 2\% Triton X-100, $50 \mathrm{U} / \mathrm{ml}$ Pierce ${ }^{\mathrm{TM}}$ Universal Nuclease, $\mathrm{pH}$ 8.0, incubating for 20 min at $37{ }^{\circ} \mathrm{C}$. The lysate was then supplemented with 0.05 volumes of sodium deoxycholate ( $10 \%$ solution in water) and further incubated at $37^{\circ} \mathrm{C}$ for $10 \mathrm{~min}$. Following the addition of 0.1 volumes of $5 \mathrm{M} \mathrm{NaCl}$, the lysate was clarified by centrifugation at 4,000 $\mathrm{xg}$ for $20 \mathrm{~min}$ at $20^{\circ} \mathrm{C}$. The cleared lysate was layered on top of a discontinuous sucrose density gradient generated by layers of $60 \%, 40 \%, 25 \%$, and $15 \%$ sucrose in $20 \mathrm{mM}$ 
diethanolamine hydrochloride, pH 9.0. Following centrifugation at 104,000 x $g$ (Beckman Optima LE-80) for $3 \mathrm{~h}$ at $20^{\circ} \mathrm{C}$, the virus was recovered from the $40 \%$ sucrose layer and the $40-60 \%$ sucrose interface region of the gradient. The AAV particles were further purified by ion exchange chromatography on a POROS 50 HQ column (ThermoFisher Scientific). AAV elution was monitored by measuring the absorbance at both 260 and $280 \mathrm{~nm}$. Fractions containing the AAV particles were buffer exchanged into phosphate buffered saline containing $0.01 \%$ Pluronic F-68, filter-sterilized, and quantified using the agarose gel method previously described (28).

Animal studies

Mice were fed a standard chow diet (Tekland 2018S) and maintained at a room temperature of 72.0 $\pm 0.3^{\circ} \mathrm{F}$, room humidity of $40 \% \pm 2 \%$, and a 12 -h light, 12 -h dark cycle, with the dark cycle starting at 18:00 h. Six-week-old C57BL/6J male mice were purchased from Jackson Laboratory. Following a 2week acclimation period, these mice were injected with $2.5^{*} 10^{11}$ genome copies of GFP- or Nudt7-AAV in $150 \mu \mathrm{l}$ of sterile phosphate buffered saline and, unless otherwise indicated, were sacrificed 3-4 weeks after injection. Unless otherwise stated, fasting experiments were started at 7:00 am, and mice were placed on cages with grids without food for the indicated amount of time. To measure food consumption, mice were individually housed in cages with grids and provided with a pre-weighed amount of food. Seventytwo hours later, the leftover food was weighed and used to calculate the average food consumption per mouse per $24 \mathrm{~h}$. Serum levels of aspartate aminotransferase (AST) and alanine aminotransferase (ALT) were measured using Stanbio kits (EKF Diagnostic USA) and 3-hydroxybutyrate was determined using a 3-hydroxybutyrate enzyme solution and colorimetric detector from Cayman Chemicals, as per manufacturer's instructions. All studies were approved by the Institutional Animal Care and Use Committees of West Virginia University.

\section{CoA analysis, immunoblotting and RT-PCR}

The concentration of total CoA (free CoA plus CoA thioesters) in liver homogenates was determined after conversion of the cofactor to the mBB derivative, as previously described (24). For western blot analysis, flash frozen tissues were homogenized in ice-cold radioimmunoprecipitation assay buffer 
supplemented with protease inhibitors (Biotool), and centrifuged at $10,000 \mathrm{x} \mathrm{g}$ for $10 \mathrm{~min}$ at $4{ }^{\circ} \mathrm{C}$. Proteins (5 or $20 \mu \mathrm{g}$ ) were fractionated on $4-12 \%$ bis-Tris polyacrylamide gels and transferred onto nitrocellulose membranes using an iBlot dry transfer system (Thermo Fisher Scientific). The GAPDH and Nudt7 antibodies were used at a 1:5,000 and 1:3,000 dilution, respectively. Bound primary antibodies were detected by chemiluminescence with HRP-conjugated goat anti-rabbit IgG at a 1:45,000 dilution. The HRP-conjugated GFP antibody was used at a 1:7,000 dilution. RNA was isolated from flash frozen tissue as previously described (29). Following the removal of genomic DNA with Turbo DNA-free kit (Thermo Fisher Scientific), mRNA levels were quantified in triplicate by RT-PCR using the Quantitect SYBR Green RT-PCR kit (Qiagen) and the primers previously reported (29). The relative abundance of each gene was calculated using the $\mathrm{C}_{\mathrm{T}}$ method, and the amount $\left(2^{-\Delta \mathrm{CT}}\right)$ reported relative to the average of ribosomal protein L22 (Rp122) and 32-microglobulin (B2m). Primers 5'-GTGCCTTTCTCCAAAAGGTATTT-3' and 5'CTCTCTTTGCTGTTGGCGAC-3' were used as forward and reverse primers, respectively, to amplify Rpl22. Primers 5'-ACTGACCGGCCTGTATGCTA-3' and 5'-ATGTTCGGCTTCCCATTCTCC-3' were used as forward and reverse primers, respectively for B2m.

RNA-Seq

Total RNA from mouse liver tissue was isolated as described above and delivered to the WVU Genomics Core Facility for quality control and library preparation. The concentration of extracted total RNA was determined using a Qubit fluorometer (Thermo Fisher Scientific) while RNA quality was ascertained on an Agilent 2100 Bioanalyzer using an RNA Nano 6000 chip. All of the samples used for the library preparation had RIN (RNA Integrity Number) values > 8.0. Each library was built using 500 ng of RNA as input material for the stranded mRNA library preparation kit from KAPA Biosystems, following the manufacturer's recommended protocol with 9 cycles of PCR. The cDNA libraries were quantified via Qubit, pooled in equimolar ratios, and sent to the Genomics Core Facility at Marshall University where the libraries were sequenced on 4 lanes generating PE50bp reads with the Illumina HiSeq1500 system (16$36 \mathrm{M}$ reads per sample, $\sim 175 \mathrm{M}$ total reads). Read quality was assessed using FastQC 
(https://www.bioinformatics.babraham.ac.uk/projects/fastqc) and deemed to require no trimming. The reads were quantified using Salmon (30) with transcripts obtained from ensemble, version GRCm38.84. Using tximport (31), the data was imported into R and differential expression was computed using DESeq2 (32). We removed all genes that did not have at least a count of 10 in at least 3 samples. Genes were considered to be significantly changed if the Benjamini-Hockburg adjusted p-value was less than 0.01. WebGestalt (http://www.webgestalt.org) was used to conduct gene ontology and pathway enrichment analyses. The RNA-Seq raw data are deposited and accessible through NCBI Gene Expression Omnibus (https://www.ncbi.nlm.nih.gov/geo/) with the GEO Series accession number GSE125237.

\section{Enzymatic assays and lipid analyses}

Total liver CoA diphosphohydrolase activity was measured in liver extracts obtained by homogenizing flash-frozen tissue ( 20 mg) in $750 \mu$ of $20 \mathrm{mM}$ Tris-HCl pH 8.0. The homogenates were incubated on ice for $10 \mathrm{~min}$ before centrifuging at 20,000 $\mathrm{x} g$ for $10 \mathrm{~min}$. The supernatants were removed and increasing amounts of protein (6.25-50 $\mu \mathrm{g}$ ) were incubated with $100 \mu \mathrm{M}$ of mBB-CoA for $10 \mathrm{~min}$ at $37^{\circ} \mathrm{C}$. The reactions were then stopped and analyzed as described previously (18).

Serum cholesterol and triglycerides were measured using Stanbio kits (EKF Diagnostic USA), as per the manufacturer's instructions. Liver lipids were extracted from $\sim 50 \mathrm{mg}$ of liver using a modification of the Bligh and Dyer procedure (33) optimized for lipid quantitation by the LipidMaps group (34). Following extraction, a portion of the lipids was dried and resuspended in $100 \mu \mathrm{l}$ of $5 \% \mathrm{NP}-40$ for cholesterol analysis using the same Stan-Bio kit utilized for the determination of cholesterol in the serum. A second portion of the total lipid extract was fractionated for triglyceride analysis on HPTLC silica plates developed in hexane:ether:acetic acid (80:20:1, v:v:v). Resolved lipid bands and serial dilutions of a tripalmitin standard were visualized and quantitated by spraying with a $0.002 \%$ primulin solution followed by fluorescence analysis using G:BOX Chemi XX9 imaging system (Syngene). 


\section{Targeted and untargeted metabolomics}

Global metabolomics profiling, combined with statistical and pathway enrichment analyses, were conducted by Metabolon, Inc. Raw data and metabolite fold-changes between groups are reported in supplemental Table 3.S1. Principal component analysis, normalization, and scaling of selected metabolites for the generation of heat plots were conducted using MetaboAnalyst 4.0 (https://www.metaboanalyst.ca/) (35). Liver, small intestine, and bile samples were analyzed for their bile acid composition using a targeted assay by Metabolon, Inc. For the analysis of the acyl-CoA pool composition, CoA species were extracted as described by Minkler P. E. et al. (36). Briefly, flash-frozen livers ( $\sim 50 \mathrm{mg})$ were homogenized in 1.5 $\mathrm{ml}$ of a 3:1 (v:v) mixture of acetonitrile:isopropanol containing $0.625 \mu \mathrm{M}$ of heptanoyl- and heptadecanoylCoA standards. Following the addition of $0.5 \mathrm{ml}$ of $0.1 \mathrm{M} \mathrm{KH}_{2} \mathrm{PO}_{4}, \mathrm{pH} 6.7$, the homogenates were centrifuged at $16,000 \times \mathrm{g}$ for $5 \mathrm{~min}$. The supernatants were acidified by the addition of 0.25 volumes of glacial acetic acid and applied onto 2-(2-pyridyl)ethyl-functionalized silica columns (100 mg, SigmaAldrich), previously conditioned with $1 \mathrm{ml}$ of a 9:3:4:4 (v:v) mixture of acetonitrile:isopropanol:water:acetic acid. The columns were washed with $1 \mathrm{ml}$ of the same solvent mixture and $1 \mathrm{ml}$ of water before eluting the bound compounds with $4 \mathrm{ml}$ of $90 \%$ methanol containing $15 \mathrm{mM}$ ammonium hydroxide. The samples were dried at room temperature under nitrogen flow and stored at -20 ${ }^{\circ} \mathrm{C}$ until analyzed. The LC/MS/MS analysis was conducted in the Metabolome Analysis Facility at West Virginia University on an AB Sciex QTrap 5500 mass spectrometer connected to an AB Sciex Exion UPLC and controlled by Analyst ${ }^{\circledR}$ Version 1.6 .3 software. Frozen extracts were re-suspended in $300 \mu \mathrm{l}$ of $50 \%$ methanol and fractionated at $0.4 \mathrm{ml} / \mathrm{min}$ onto an Acquity BEH amide column (2.1 x $50 \mathrm{~mm}, 1.7 \mu \mathrm{m}$ particle size, Waters) kept at $25{ }^{\circ} \mathrm{C}$ and equilibrated with $10 \%$ solvent $\mathrm{A}$ (50 mM ammonium formate, $\mathrm{pH}$ 9.5) and $90 \%$ solvent B (acetonitrile). Following the injection of $1.5 \mu \mathrm{l}$ of sample, the concentration of A was maintained at $10 \%$ for an additional $1.5 \mathrm{~min}$, followed by a linear gradient that increased the concentration of $\mathrm{A}$ to $50 \%$ in $6.5 \mathrm{~min}$ and then to $65 \%$ in $30 \mathrm{sec}$. The concentration of A was kept at $65 \%$ for $1.5 \mathrm{~min}$ before returning it to $10 \%$ in $3.5 \mathrm{~min}$, followed by $4.5 \mathrm{~min}$ of column re-equilibration. Positive ESI-MS/MS 
was performed using the following parameters: ionspray voltage of $5.5 \mathrm{kV}$, temperature of $500{ }^{\circ} \mathrm{C}$, ion source gas 1 set at 25 , ion source gas 2 and curtain gas set at 20, collision gas set at medium. The entrance potential (EP), collision energy (CE), and collision cell exit potential (CXP) resulting in the neutral loss of 507.0 Da from the $(\mathrm{M}+\mathrm{H})^{+}$precursor $(37,38)$ were individually optimized for free CoA $(\mathrm{EP}=10 \mathrm{~V}, \mathrm{CE}=$ $37 \mathrm{~V}, \mathrm{CXP}=15 \mathrm{~V})$, acetyl-CoA $(\mathrm{EP}=10 \mathrm{~V}, \mathrm{CE}=40 \mathrm{~V}, \mathrm{CXP}=15 \mathrm{~V})$, malonyl-CoA $(\mathrm{EP}=12 \mathrm{~V}, \mathrm{CE}=40$ $\mathrm{V}, \mathrm{CXP}=15 \mathrm{~V})$, succinyl-CoA $(\mathrm{EP}=10 \mathrm{~V}, \mathrm{CE}=45 \mathrm{~V}, \mathrm{CXP}=17 \mathrm{~V})$, butyryl-CoA $(\mathrm{EP}=12 \mathrm{~V}, \mathrm{CE}=41$ $\mathrm{V}, \mathrm{CXP}=20 \mathrm{~V})$, heptanoyl-CoA $(\mathrm{EP}=10 \mathrm{~V}, \mathrm{CE}=45 \mathrm{~V}, \mathrm{CXP}=20 \mathrm{~V})$, octanoyl-CoA $(\mathrm{EP}=7 \mathrm{~V}, \mathrm{CE}=46$ $\mathrm{V}, \mathrm{CXP}=23 \mathrm{~V})$, lauroyl-CoA $(\mathrm{EP}=9 \mathrm{~V}, \mathrm{CE}=47 \mathrm{~V}, \mathrm{CXP}=20 \mathrm{~V})$, heptadecanoyl-CoA $(\mathrm{EP}=10 \mathrm{~V}, \mathrm{CE}$ $=47 \mathrm{~V}, \mathrm{CXP}=20 \mathrm{~V})$, and stearoyl-CoA $(\mathrm{EP}=9 \mathrm{~V}, \mathrm{CE}=50 \mathrm{~V}, \mathrm{CXP}=23 \mathrm{~V})$. The declustering potential was consistently set at 170 volts. For all other CoA species, the parameters EP, CE and CXP were set at 10, 45 and $17 \mathrm{~V}$, respectively. Retention times for the detected CoA species are reported in supplementary Table 3.S2. Peak analysis was conducted using MultiQuant 3.0.2. Peak areas corresponding to free CoA and acyl-CoAs with an acyl chain up to 10 carbons were normalized to the heptanoyl-CoA internal standard. Peak areas corresponding to longer acyl-CoAs and choloyl-CoA were normalized to the heptadecanoylCoA internal standard. Corrected peak areas were further normalized to the tissue weight.

\section{Hepatocyte isolation}

For immunofluorescence studies, primary hepatocytes were isolated from fed mice using the twostep liver perfusion method described by Klaunig et al. (39) with minor modifications. All perfusion steps were conducted at a flow rate of $7 \mathrm{ml} / \mathrm{min}$ with buffers kept at $37^{\circ} \mathrm{C}$. The livers were first perfused with $50 \mathrm{ml}$ of a modified Krebs buffer $(120 \mathrm{mM} \mathrm{NaCl}, 24 \mathrm{mM} \mathrm{NaHCO}$, $20 \mathrm{mM}$ glucose, $5 \mathrm{mM}$ HEPES, 4.8 $\mathrm{mM} \mathrm{KCl}, 1.2 \mathrm{mM} \mathrm{MgSO} 4,1.2 \mathrm{mM} \mathrm{KH} \mathrm{PO}_{4}, 100 \mathrm{U} / \mathrm{ml}$ penicillin, $100 \mathrm{U} / \mathrm{ml}$ streptomycin, $50 \mu \mathrm{g} / \mathrm{ml}$ gentamycin) containing 0.1 mM EGTA, pH 7.4, followed by 50 ml of modified Krebs buffer supplemented with $1.4 \mathrm{mM} \mathrm{CaCl}_{2}$ and $10 \mu \mathrm{g} / \mathrm{ml}$ of Liberase TM (Sigma-Aldrich). Intermittent clamping of the inferior vena cava for about 10 sec each time was conducted during this latter step to increase total cell yield (40). After the perfusion, the livers were excised, separated from the gall bladder, and transferred to a sterile dish containing $20 \mathrm{ml}$ of the second perfusion buffer, ice-cold, without Liberase. The cells were released by 
tearing the liver with forceps. The cell suspension was filtered through a $100 \mu \mathrm{m}$ strainer (Fisher Scientific) and washed 3 times with Waymouth medium (Waymouth MB 752/1 medium supplemented with $0.5 \mathrm{mM}$ serine, $0.5 \mathrm{mM}$ alanine, $20 \mathrm{mM}$ HEPES, pH 7.4, $100 \mathrm{U} / \mathrm{ml}$ penicillin, $100 \mathrm{U} / \mathrm{ml}$ streptomycin, 50 $\mu \mathrm{g} / \mathrm{ml}$ gentamycin) containing $1.9 \mathrm{mg} / \mathrm{ml}$ of fatty acid-free bovine serum albumin and pelleted by spinning at 50 $\mathrm{x} g$ for $2 \mathrm{~min}$ at $4^{\circ} \mathrm{C}$. After the washes, the cells were re-suspended in Waymouth medium containing $5 \%$ newborn calf serum, and $7.5 * 10^{5}$ cells were plated on each well of collagen-coated 6-well plates. Viability (consistently $>85 \%$ ) and yield were assessed by counting the cells that excluded trypan blue. After a $5 \mathrm{~h}$ incubation, the medium was replaced with fresh Waymouth medium lacking the newborn calf serum and an overlay of Matrigel $(0.2 \mathrm{mg} / \mathrm{ml})$ and insulin $(60 \mathrm{nM})$ was added as described by Cyphert, H. A. et al. (41). The hepatocytes were incubated under these conditions overnight before being processed for immunofluorescence studies.

For the measurement of the rate of $\beta$-oxidation, the procedure for the isolation of hepatocytes was modified as follows. First, hepatocytes were isolated from mice fasted for $15 \mathrm{~h}$ (food removed at 4:00 pm) using perfusion buffers containing a reduced amount of glucose at $5.5 \mathrm{mM}$. Second, after releasing the cells from the liver capsule, M199 containing 25 mM of HEPES, pH 7.4 (M199/HEPES) was used for all the wash steps and to re-suspend the cells at a final concentration of $1.0 * 10^{6}$ cells $/ \mathrm{ml}$. This cell suspension was immediately used for $\beta$-oxidation studies.

\section{Immunofluorescence and $\beta$-oxidation}

Confocal microscopy was used as previously described (18) to determine the subcellular localization of the exogenous FLAG-tagged Nudt7 expressed in primary hepatocytes isolated from mice injected with Nudt7-AAV. The DYKDDDK antibody, the PMP70 antibody used to visualize the peroxisomes, and an Alexa Fluor 647 donkey-anti rabbit secondary antibody were used at a 1:1,000

dilution. $\beta$-Oxidation studies were conducted in triplicate on freshly isolated hepatocytes in suspension by measuring the production of ${ }^{14} \mathrm{C}$-labeled acid-soluble metabolites from $\left[1-{ }^{14} \mathrm{C}\right]$-palmitic acid (American Radiolabeled Chemicals) in the presence or absence of etomoxir, an inhibitor of mitochondrial fatty acid oxidation. Pilot experiments were conducted to determine the optimal combination of cell number and 
incubation time that would ensure linear product formation. Furthermore, pilot studies comparing the delivery of $\left[1-{ }^{14} \mathrm{C}\right]$-palmitic acid as a solution in ethanol or as a dry powder thoroughly resuspended in M199/HEPES containing 0.5\% fatty acid-free bovine serum albumin ruled out any adverse effect of $17 \mathrm{mM}$ final ethanol concentration on the rate of fatty acid oxidation. Specifically, $7.5 * 10^{5}$ cells were pre-incubated for $15 \mathrm{~min}$ at $37{ }^{\circ} \mathrm{C}$ in $1.5 \mathrm{ml}$ of M199/HEPES $\pm 45 \mu \mathrm{M}$ etomoxir before starting the reaction with the addition of $0.5 \mathrm{ml}$ of substrate mixture containing $0.5 \%$ fatty acid-free bovine serum albumin and $0.4 \mathrm{mM}$ of $\left[1-{ }^{14} \mathrm{C}\right]$-palmitic acid $(0.4 \mu \mathrm{Ci}$, specific activity $2 \mathrm{mCi} / \mathrm{mmol}$, from a $111 \mathrm{mM}$ stock solution in ethanol) in M199/HEPES. The hepatocytes were incubated with gentle shaking for $15 \mathrm{~min}$ at $37^{\circ} \mathrm{C}$ before stopping the reaction by transferring $400 \mu \mathrm{l}$ of cell suspension to a tube containing $133 \mu \mathrm{l}$ of $1 \mathrm{M}$ perchloric acid. This acidified suspension was centrifuged at 20,000 x $\mathrm{g}$ for $10 \mathrm{~min}$ and the radioactivity in $300 \mu \mathrm{l}$ of supernatant was measured by liquid scintillation counting. Typical raw counts in samples incubated without etomoxir were between 4,000 and 9,000 cpm, while typical raw counts in samples incubated with etomoxir were between 1,500 and 3,000 cpm. Background radioactivity (350-700 cpm), estimated in reactions stopped immediately after the addition of the substrate mixture, was subtracted from each sample. For each mouse, peroxisomal $\beta$-oxidation was calculated from the samples incubated with etomoxir. Mitochondrial fatty acid oxidation was calculated as the difference between total fatty acid oxidation (no etomoxir) and peroxisomal fatty acid oxidation.

Statistical analysis

All data are reported as the mean with \pm the standard error. Unless otherwise stated, statistical significance $(p<0.05)$ was calculated using the Welch's two-sample t-test (Metabolon Inc.) or the unpaired two-tailed Student's t-test and the two-way ANOVA using GraphPad Prism 7 (GraphPad Software).

\section{$\underline{\text { Results }}$}

Overexpression of Nudt7 does not prevent the increase in total CoA levels that occurs in fasted livers

Compared to fed mice, the livers of mice fasted for 24 or 48 h exhibit a higher concentration of total CoA (free CoA plus acyl-CoAs) and lower expression of the CoA-degrading enzyme Nudt7, the major 
CoA diphosphohydrolase isoform in this organ $(17,18,22)$. To examine the inverse correlation between the cofactor concentration and Nudt7 expression in more detail, we measured liver CoA levels and Nudt7 mRNA and activity during a fasting time course, starting as early as $6 \mathrm{~h}$ after the removal of food (Fig. 3.1). We found that CoA levels increased significantly after a $12 \mathrm{~h}$ fast (Fig. 3.1A) and plateaued after a $24 \mathrm{~h}$ fast, closely matching the time course of the decrease in Nudt7 mRNA (Fig. 3.1B) and CoA-degrading activity (Fig. 3.1C) in the liver. To determine whether suppression of Nudt7 activity contributed to this accumulation of CoA, we overexpressed Nudt7 with an N-terminal FLAG tag in the livers of C57BL/6J mice and analyzed the effect of this manipulation on the concentration of hepatic CoA in fasted mice (Fig. 3.2). Transgene expression was obtained using AAV particles carrying the Nudt7 coding sequence under the control of the liver-specific LP1 promoter (42). Mice expressing GFP from the same AAV construct were used as controls and to characterize the transgene expression from this system, which was stable for up to 12 weeks (Fig. 3.2A) and confirmed to be liver-specific (Fig. 3.2B). All analyses were conducted 34 weeks after the AAV injection. Under these conditions, values for serum AST and ALT were in the normal range (43) and similar between GFP and Nudt7 mice, indicating the absence of liver damage (Table 3.1). Furthermore, injection of Nudt7-AAV did not affect food intake or body weight and the weight of the liver normalized to body weight was modestly elevated in the Nudt7 mice under fed but not fasting conditions (Table 3.1). Exogenous Nudt7 was robustly overexpressed compared to the endogenous levels (Fig. 3.2C, inset) and measurement of the CoA-degrading activity in Nudt7 mice fasted for $24 \mathrm{~h}$ revealed a 10-fold increase in activity compared to either GFP mice or mice that were not injected with AAV (Fig. 3.2C). Nudt7 is a peroxisomal enzyme $(23,24)$. To determine whether the exogenously expressed Nudt7 was correctly localized to the peroxisomes, we isolated primary hepatocytes from mice injected with Nudt7AAV and used immunofluorescence and confocal microscopy to visualize the subcellular localization of the FLAG epitope fused to the transgene. Nuclei were stained with DAPI and peroxisomes were visualized with an antibody against the endogenous protein PMP70. FLAG-tagged Nudt7 was found to co-localize with PMP70 with no detectable fluorescence in the cytoplasm (Fig. 3.2D), confirming efficient import of this protein into the peroxisomes. Nudt7 and GFP mice were then analyzed for total hepatic CoA levels in 
the fed state and following a 24 and $48 \mathrm{~h}$ fast. The hepatic concentration of CoA tended to be lower in fed and 24 h fasted Nudt7 mice compared to the GFP controls but the difference did not reach statistical significance (Fig. 3.2E). Furthermore, total CoA levels increased with fasting in both Nudt7 and GFP livers, indicating that overexpression of Nudt7 was not able to blunt the accumulation of CoA driven by activation of the biosynthetic pathway. Overall, these results supported the conclusion that the fastinginduced decrease in Nudt7 activity was unlikely to significantly contribute to the increase in the total CoA content normally observed, at the whole tissue level, in the liver (Fig. 3.1).

Increased Nudt7 activity in the fasted state changes the acyl-CoA composition and affects lipid metabolism in the liver

Nudt7 is a promiscuous CoA diphosphohydrolase that hydrolyzes free CoA and a large range of short- and medium-chain acyl-CoAs in vitro $(17,18)$. To determine whether overexpression of Nudt7 changed the concentration of individual acyl-CoA species, we analyzed the relative composition of the acyl-CoA pool in Nudt7 and GFP livers by LC/MS/MS.

We found that, upon fasting, the vast majority of the short-chain acyl-CoAs monitored were significantly decreased in Nudt7 mice compared to the GFP controls (Fig. 3.3D-F). These included CoA thioesters known to be excellent in vitro substrates for Nudt7, such as acetyl-, propionyl-, (iso)butyryl-, hexanoyl-, malonyl-, and succinyl-CoA. Acetoacetyl-, 3-hydroxyl(iso)butyryl, crotonyl/methacryloyl- and tyglyl/methylcrotonyl-CoA, which are products of fatty acid and amino acid metabolism, were also decreased in the Nudt7 livers (Fig. 3.3E). The drop in the concentration of free CoA observed in fasted Nudt7 livers narrowly missed statistical significance (Fig. 3.3D). Choloyl-CoA, an intermediate in bile acid synthesis and known substrate of recombinant Nudt7 (17), was significantly decreased by Nudt7 overexpression in the fasted state and tended to be lower even in the fed state (Fig. 3.3C and F). Overexpression of Nudt7 had minimal effects on the acyl-CoA composition in the fed state, with adipoylCoA, a six-carbon dicarboxylic fatty acyl-CoA, being the only CoA thioester significantly decreased in fed Nudt7 mice compared to the GFP controls (Fig. 3.3A-C). Adipoyl-CoA levels remained significantly lower in the Nudt7 livers following a $24 \mathrm{~h}$ fast. 
Combined, these results showed that while the overexpression of Nudt7 did not significantly affect total CoA levels, it changed the acyl-CoA composition of the liver by decreasing the concentration of shortchain acyl-CoAs and choloyl-CoA. This effect was more pronounced in the fasted state, when the expression and activity of the endogenous Nudt7 in the liver were lowest (Fig. 3.1B and C).

Mono and dicarboxylic short-chain acyl-CoAs are products and intermediates of a variety of metabolic processes, including lipid, carbohydrate, and amino acid metabolism. To identify pathways that could be potentially altered by Nudt7 overexpression, we conducted global metabolic profiling of Nudt7 and GFP livers harvested from fed and fasted mice (Fig. 3.4, supplementary Table 3.S1). As expected, the vast majority of the 769 metabolites detected was changed by the nutritional state, regardless of the specific transgene expressed (Fig. 3.4A). The separation between the fed and fasted mice, together with the significant overlap between GFP and Nudt7 groups, was also highlighted by principal-component analysis (Fig. 3.4B). In the fed state, 66 metabolites were significantly different between Nudt7 and GFP livers, while this number almost doubled to 111 in the fasted state (Fig. 3.4A). Only a few CoA species, CoA precursors and metabolites were detected by the untargeted metabolic profiling. Among the latter were the products of the Nudt7 reaction, phosphopantetheine and 3',5'-ADP, which were not significantly different between Nudt7 and GFP livers, although the higher levels of phosphopantetheine trended toward significance in the fasted Nudt7 livers (supplementary Table 3.S1). The larger effect of Nudt7 expression on liver metabolism in the fasted state was consistent with the results from the acyl-CoA analysis (Fig. 3.3E) and led us to focus all subsequent analyses on this nutritional state. Under fasting conditions, pathway enrichment analysis revealed that the 3 most significantly and highly (>3-fold) enriched pathways were primary and secondary bile acid metabolism, henceforth considered as a single pathway, and acylcarnitines (Fig. 3.4C). These pathways, which are both part of lipid metabolism and either dependent on or tightly linked to CoA, were further analyzed (see below).

We also conducted RNA-Seq analysis of the livers of fasted Nudt7 and GFP mice. This analysis revealed a modest effect of Nudt7 overexpression on the global transcriptome, identifying a total of 62 genes (33 upregulated, 29 downregulated) whose expression was significantly different in the Nudt7 livers 
compared to the GFP controls (fold-change $>1.50$ or $\leq 0.66$, adjusted $p$ value $<0.01$ ) (supplementary Table 3.S3). Gene ontology analysis on this list of differentially expressed genes identified carboxylic ester hydrolase activity and fibronectin binding as significantly enriched molecular functions (Table 3.2), but no significantly enriched biological process. Pathway analysis using the Wikipathway database identified the PPAR signaling pathway as significantly enriched (Table 3.2). The same result was obtained using the KEGG database.

Effect of increased Nudt7 activity on peroxisomal bile acid metabolism

A heat plot of all the primary and secondary bile acids detected by the untargeted metabolomics analysis is shown in Fig 3.5A. Out of the 23 bile acids quantified, 20 were significantly decreased in the livers of Nudt7 mice, with no difference in the levels of the bile acid precursors cholesterol and $7 \alpha-$ hydroxycholesterol (44). The concentration of liver and serum cholesterol was also confirmed to be similar between GFP and Nudt7 mice by an independent assay (Table 3.1). To corroborate the results obtained from the untargeted metabolomics analysis, we injected another cohort of mice with GFP- and Nudt7-AAV and examined the bile acid composition of their livers with a targeted bile acid assay. This analysis was also extended to the gall bladder and small intestine of the mice, as these organs contain a large portion of the body bile acid pool. In the Nudt7 livers, the targeted analysis confirmed a significant $\sim 50 \%$ decrease in selected primary and secondary bile acids including tauro- $\alpha$-muricholic acid, taurocholic acid, taurodeoxycholic acid and tauroursodeoxycholic acid compared to the GFP controls (Fig. 3.5B). Hepatic levels of $\beta$-muricholic acid and tauro- $\beta$-muricholic acid also tended to be lower in the Nudt7 mice but did not reach statistical significance in the targeted analysis. No difference in the bile acid composition of the gall bladder and small intestine was detected (Fig. 3.5C and D), indicating that the effect of the increased activity of Nudt7 was limited to the liver, the site of bile acid synthesis and Nudt7 overexpression.

Conversion of the primary bile acid precursors trihydroxycholestanoic acid (THCA) and dihydroxycholestanoic acid (DHCA) to cholic and chenodeoxycholic acid, respectively, requires their activation as CoA thioesters and import into the peroxisomes, where their branched side chain undergoes a 
cycle of $\beta$-oxidation (14) (Fig. 3.5E). This process requires free CoA and releases propionyl-CoA and either chenodeoxycholoyl-CoA (CDCA-CoA, from DHCA-CoA) or choloyl-CoA (from THCA-CoA), which are then primarily conjugated to taurine in mice. In the Nudt7 livers, the observed drop in the concentration of cholic acid and its derivatives was consistent with a robust decrease in the levels of choloyl-CoA (Fig. 3.3F). Similar to choloyl-CoA, THCA-CoA is a known substrate of Nudt7 (17); however, the concentration of THCA-CoA was below the detection limit of our assay and we could not determine whether the Nudt7 livers contained less of this bile acid precursor as well. At the gene expression level, Cyp39a1 was the only gene involved in bile acid metabolism that was differentially expressed and, specifically, downregulated, in the Nudt7 livers (supplementary Table 3.S3). Cyp39a1 encodes a microsomal cytochrome P450 enzyme that possesses $7 \alpha$-hydroxylase activity against 24(S)hydroxycholesterol (45), but the contribution of this enzyme to bile acid synthesis in the liver is currently unknown.

Effect of Nudt7 overexpression on fatty acid $\beta$-oxidation

In addition to bile acids, the metabolic profiling of the livers identified 18 acylcarnitines that were significantly changed between Nudt7 and GFP mice in the fasted state (Fig. 3.6A). Acylcarnitines are in close equilibrium with acyl-CoAs due to the activity of carnitine acyltransferases that transfer the fatty acid from an acyl-CoA to free carnitine to prevent a buildup of acyl-CoAs at the expense of free CoA (46). Among the acylcarnitines significantly changed by the overexpression of Nudt7, short-chain acylcarnitines such as propionylcarnitine, succinylcarnitine, glutarylcarnitine and adipoylcarnitine were significantly decreased, correlating with the global drop in the concentration of short-chain acyl-CoAs observed in the Nudt7 livers (Fig. 3.3E). Conversely, acylcarnitines with acyl chains between 8 and 20 carbons in length ( $\mathrm{C}_{8-20}$-acylcarnitines) were found to accumulate, suggesting increased transfer of medium and long-chain acyl groups from the correspondent acyl-CoAs to free carnitine. The concentration of free carnitine, which is the most abundant of the carnitine species in the liver, was not affected by these changes in the composition of the acylcarnitine pool $(16,47,48)$. 
Since this metabolic signature was consistent with a potential decrease in fatty acid oxidation $(16,49,50)$, we measured the rate of ${ }^{14} \mathrm{C}$-labeled palmitic acid oxidation in primary hepatocytes isolated from fasted Nudt7 and GFP mice. To discriminate between the mitochondrial and peroxisomal pathways, we incubated the hepatocytes with and without etomoxir, an inhibitor of mitochondrial carnitine palmitoyltransferase 1 and thus, mitochondrial fatty acid oxidation. Additionally, we measured the serum concentration of 3-hydroxybutyrate and glucose, as the circulating levels of these metabolites are linked to the oxidation of fatty acids in liver mitochondria through the production of NADH and acetyl-CoA in this subcellular compartment (Table3. 1). We did not detect any significant difference in the rate of mitochondrial fatty acid oxidation between groups (Fig 3.6B), and this result correlated with similar levels of 3-hydroxybutyrate and glucose in the serum of Nudt7 and GFP mice (Table 3.1). Conversely, we found that increased Nudt7 activity reduced the rate of fatty acid oxidation in the peroxisomes (Fig. 3.6B), the subcellular compartment where Nudt7 localized. Peroxisomes are known to preferentially shorten fatty acids instead of completely oxidizing them to acetyl-CoA (51). Indeed, octanoate undergoes only 1-2 cycles of peroxisomal fatty acid oxidation to produce hexanoate and butyrate (52). Consistent with this notion, the Nudt7 livers accumulated $\mathrm{C}_{8-20}$-acylcarnitines while exhibiting a lower concentration of acylCoAs and acylcarnitines with 6 carbons or less, which are the final products of the oxidation of monocarboxylic fatty acids (hexanoyl- and butyryl-CoA) and dicarboxylic fatty acids (adipoyl- and succinyl-CoA) in the peroxisomes (Fig. 3.6C). The decrease in the levels of these short-chain acyl-CoAs could have also been further exacerbated by direct degradation of these products by exogenous Nudt7.

Overexpression of Nudt7 led to a 2-fold increase in the expression of Acaa1b, the gene encoding the peroxisomal enzyme acetyl-coenzyme A acyltransferase 1B, also known as 3-ketoacyl-CoA thiolase B (Table 3.2). Acca1b catalyzes the last, free CoA-dependent step in the oxidation of straight chain fatty acids $(25,53)$. No other gene involved in either peroxisomal or mitochondrial fatty acid oxidation was differentially expressed in the Nudt7 livers. Although peroxisomal fatty acid oxidation was blunted in the Nudt7 livers, hepatic triglyceride levels were similar between Nudt7 and GFP mice (Table 3.1). 
Interestingly, this was associated with the upregulation of genes involved in the hydrolysis of triglycerides (Ces1g, Ces1f and Ces2c) (54-56) and monoacylglycerols (Abhd6) (57-59) (Table 3.2).

Taken together with the effect on bile acid metabolism, the above data indicated that overexpression of Nudt7 predominantly affected peroxisomal lipid metabolism.

\section{$\underline{\text { Discussion }}$}

In this study, we determined the metabolic consequences of increasing the CoA-degrading activity in the liver by overexpressing mouse Nudt7, the most abundant of the currently known CoA diphosphohydrolases in this organ $(17,18,22)$. One of our major findings was that the overexpression of the enzyme did not interfere with the increase in total liver CoA levels that occurs in the transition from the fed to the fasted state (Fig. 3.2E). This supports the conclusion that, upon fasting, the rise in CoA observed at the whole tissue level is driven by increased synthesis (16), with negligible contribution from a decrease in Nudt7 expression and activity (Fig. 3.1B and C).

The exogenously expressed Nudt7 was correctly localized to the peroxisomes (Fig. 3.2D). Based on subcellular fractionation studies on rat livers, these organelles are estimated to contain only about 4-10\% of the total hepatic CoA, depending on the nutritional state $(11,60)$. This subcellular localization combined with the fact that overexpression of the enzyme had no significant effect on total liver CoA levels but decreased the concentration of choloyl-CoA, a CoA thioester specifically generated in the peroxisomes, suggests that the activity of this enzyme may have been restricted to the peroxisomal CoA pool. This conclusion is further supported by the second major finding of this study: increased expression of Nudt7 in the fasted state specifically blunted peroxisomal processes, including peroxisomal fatty acid $\beta$-oxidation (Fig. 3.6).

Overexpression of Nudt7 in the fed state, when the endogenous Nudt7 levels are highest, had a very modest effect on the composition of the hepatic acyl-CoA pool. Conversely, overexpression of Nudt7 in the fasted state led to a $15-50 \%$ decrease in the concentration of most of the short-chain acyl-CoAs detected (Fig. 3.3E). Short-chain acyl-CoAs, which comprise a small portion of total liver CoA $(15,60,61)$, 
are excellent substrates for the enzyme $(17,18)$; thus, increased degradation of these CoA species likely contributed to lowering their concentration in the Nudt7 livers. However, this mechanism alone would not explain the distinctive accumulation of $\mathrm{C}_{8-10}$-acylcarnitines observed in the same samples (Fig. 3.6A). This metabolic signature was associated with a reduced rate of peroxisomal fatty acid oxidation, which, in turn, likely blunted product formation. Among the products whose concentrations were curtailed were hexanoyland butyryl-CoA, which derive from the shortening of straight-chain fatty acids, acetyl-CoA, which is produced at each cycle of fatty acid oxidation, and both adipoyl- and succinyl-CoA, which are the final products of the oxidation of long-chain dicarboxylic fatty acids, a process that preferentially occurs in the peroxisomes (52,62-64). Propionyl-CoA, whose concentration was also significantly decreased in the fasted Nudt7 livers, can be produced from the oxidation of 2-methyl-branched-chain fatty acids, another process that is restricted to the peroxisomes (14). The accumulation of acylcarnitines with a wide range of acyl chain lengths also suggests that the flux through the whole peroxisomal $\beta$-oxidation pathway, and not just at a specific step, was decreased in the Nudt7 livers. This, in turn, correlated with a strong trend towards a lower concentration of free CoA, which is required to complete each cycle of fatty acid oxidation and might have been limiting in the peroxisomes of the Nudt7 livers due to increased hydrolysis (Fig. 3.3D). Analysis of the acyl-CoA pool composition in the peroxisomes would be required to confirm this hypothesis; however, the measurement of individual CoA species, and even total CoA, in mitochondriafree peroxisomal fractions is still technically challenging in mice due to the small sample size. As observed in other mouse models, inhibition of peroxisomal fatty acid oxidation in the Nudt7 livers was also associated with the activation of PPAR $\alpha$ and with increased transcription of genes involved in lipid transport and metabolism (Table 3.2) (65,66).

Efficient off-loading of acyl chains from CoA to carnitine likely explains why, unlike medium- and long-chain acylcarnitines, no change in the concentration of the correspondent acyl-CoAs was detected in the Nudt7 livers at the whole tissue level. For octanoyl- and, possibly, decanoyl-CoA, this process could occur inside the peroxisomes by the action of carnitine octanoyltransferase (Crot), which is abundant in mouse liver (67-69). For acyl-CoAs with a $\mathrm{C}_{10}$ acyl group or longer, the formation of acylcarnitines would 
likely be catalyzed by carnitine palmitoyltransferase 1 , following hydrolysis of long-chain acyl-CoAs by peroxisomal thioesterases, such as Acot3 or Acot8, and re-activation of the released free fatty acids as acylCoAs outside the peroxisomes $(27,67,69,70)$. Since CoA species are too bulky to diffuse through the peroxisomal membrane (71), the presence of multiple peroxisomal thioesterases and Crot allows the acyl group attached to CoA to leave the peroxisomes as free fatty acids or acylcarnitines and enter metabolic pathways in other subcellular compartments. For example, acetate produced from acetyl-CoA inside the peroxisomes contributes to the synthesis of malonyl-CoA $(52,72)$, whose levels were correspondingly decreased in the Nudt7 livers. Similarly, the succinyl group derived from the oxidation of dicarboxylic fatty acids in the peroxisomes can feed into the TCA cycle (62). In the livers of the fasted Nudt7 mice, the levels of succinyl-CoA and succinylcarnitine were acutely decreased by 40 and $80 \%$, respectively. In spite of this, the concentration of succinate, fumarate, malate, and oxaloacetate was not significantly different between Nudt7 and GFP livers (supplementary Table 3.S1), indicating that anaplerosis from amino acid metabolism was sufficient to support the portion of the TCA cycle that, together with pyruvate carboxylation, produces mitochondrial oxaloacetate for gluconeogenesis. Accordingly, fasting blood glucose levels were similar between Nudt7 and GFP mice (Table 3.1). Interestingly, Taniguchi M. et al. identified an association between Nudt7 expression and the redness of pork meat (73) and subsequently showed that overexpression of porcine Nudt7, which is about $70 \%$ identical to the murine isoform, leads to a significant decrease in heme content during rat L6 myoblast differentiation (74). Heme biosynthesis starts from the condensation of succinyl-CoA and glycine in the mitochondria (75). The authors suggested that that the effect on heme levels could be due to reduced availability of succinyl-CoA, a conclusion supported by our data. Overall, our results strongly suggest that Nudt7 activity contributes to the regulation of the levels of acetyl-CoA, succinyl-CoA, and other short-chain acyl-CoAs produced by fatty acid oxidation in the peroxisomes.

In addition to peroxisomal fatty acid oxidation, Nudt7 overexpression in the fasted state also altered bile acid metabolism. In particular, the fasted Nudt7 livers contained lower amounts of both primary and secondary bile acids (Fig. 3.5). This phenotype was associated with a robust decrease in the concentration 
of choloyl-CoA (Fig. 3.3F), a bile acid precursor produced via $\beta$-oxidation of the 2-methylacyl side chain of THCA-CoA in the peroxisomes $(14,44)$. Both THCA-CoA and choloyl-CoA are known substrates for Nudt7 (17) and the decrease in the concentration of choloyl-CoA could be due to a combination of mechanisms. These include an increase in direct degradation of choloyl-CoA by Nudt7, increased degradation of THCA-CoA, and/or decreased $\beta$-oxidation of THCA-CoA, which could not be detected in our samples. No information is available on the ability of Nudt7 to hydrolyze DHCA-CoA and CDCACoA in vitro and neither compound was detected in the GFP or Nudt7 livers. In spite of this, the robust decrease in chenodeoxycholic acid derivatives, including conjugated and unconjugated $\alpha$ - and $\beta$-muricholic acids, suggests the possibility that these CoA thioesters could be Nudt7 substrates as well. Combined, the drop in the availability of choloyl-CoA and the decrease in the hepatic concentration of both cholic acid and chenodeoxycholic acid derivatives suggest that increased Nudt7 activity may have blunted bile acid synthesis by interfering with the final, peroxisomal steps of the pathway.

Once synthesized in the liver, bile acids are immediately secreted and stored in the gall bladder until food ingestion leads to the contraction of this small organ to empty the bile into the intestinal tract. Most of the bile acids are then re-absorbed in the terminal ileum, secreted into the portal blood circulation, and transported back to the liver, where the small amount of bile acids lost in the feces is replenished by de novo synthesis (76). Only about $5 \%$ of the total bile acid pool needs to be synthesized by the liver at each cycle; thus, a defect in bile acid synthesis would take several fasting-to-refeeding cycles to affect the size of the total bile acid pool. This might explain why, in the fasted Nudt7 mice, the decrease in the bile acid content was only detected at the site of synthesis but it did not affect the significantly larger pools contained in the gall bladder and small intestine (Fig. 3.5B-D).

Interestingly, Nudt7 overexpression in the liver also decreased the concentration of secondary bile acids, independent of any compositional change in the intestinal bile acid pool. Secondary bile acids are produced in the intestine by de-conjugation and de-hydroxylation of primary bile acids by the gut microbiome and arrive to the liver through the enterohepatic circulation $(44,76)$. Once in the liver, the secondary bile acids are activated as acyl-CoAs and re-conjugated to taurine or glycine. Due to the dual 
localization of the enzyme that catalyzes this amidation reaction, bile acid-CoA: amino acid Nacyltransferase (Baat), the subcellular site of the secondary bile acid conjugation has been debated to be the cytosol or, as for the primary bile acids, the peroxisomes (77-79). Since there was no detectable amount of Nudt7 in the cytosol, the decrease in the concentration of secondary bile acids suggests that at least a portion of the correspondent acyl-CoAs was imported in the peroxisomes for conjugation and was hydrolyzed by Nudt7. Combined with the ability of Nudt7 to hydrolyze choloyl-CoA, this would support the conclusion that Nudt7 and Baat compete for substrates. Bile acids are signaling molecules that bind to multiple G protein-coupled receptors and nuclear receptors, such as the farnesoid X receptor and pregnane X receptor. In spite of this, the decrease in the bile acid content of the liver did not cause any significant changes in the expression of those receptors' known targets, including small heterodimer partner (NrOb2, SHP) and cholesterol $7 \alpha$ hydroxylase (Cyp7a1) $(44,76)$ (supplementary Table 3.S3). Overall, gene expression was only modestly affected in the Nudt7 livers compared to the GFP controls, supporting the conclusion that the observed changes in peroxisomal metabolism were a direct consequence of the increased CoAdegrading activity of Nudt7.

Peroxisomal fatty acid oxidation and bile acid synthesis are normally activated in the fasted state $(80,81)$, when Nudt7 expression is at its lowest. Altogether, our data support the conclusion that modulation of Nudt7 activity by the nutritional state could contribute to the regulation of the output of both pathways. Analysis of these processes in the livers of $\mathrm{Nudt}^{-/}$mice will be required to further corroborate the role of Nudt7 in the regulation of peroxisomal lipid metabolism in this organ. Generation of the $N u d t 7^{-/}$mice has recently been reported, but the phenotyping of these mice was limited to cartilage and immature murine articular chondrocytes, which, in the presence of phytol, showed decreased levels of catalase, suggesting a connection to peroxisomal function (82). Our data also strongly suggest that Nudt7-dependent CoA degradation may be limited to the quantitatively small pool of peroxisomal CoA. Analysis of the total CoA concentration in the livers of the fed and fasted $\mathrm{Nudt}^{-/-}$mice will also be important to determine whether Nudt7, and its increased activity in the fed state, is indeed responsible for the decrease in CoA levels that occurs, at the whole tissue level, in the transition from the fasted to the fed state. 


\section{$\underline{\text { References }}$}

1. Weickert, M. O., and Pfeiffer, A. F. (2006) Signalling mechanisms linking hepatic glucose and lipid metabolism. Diabetologia 49, 1732-1741

2. Bechmann, L. P., Hannivoort, R. A., Gerken, G., Hotamisligil, G. S., Trauner, M., and Canbay, A. (2012) The interaction of hepatic lipid and glucose metabolism in liver diseases. Journal of hepatology 56, 952-964

3. $\quad$ Rui, L. (2014) Energy metabolism in the liver. Compr Physiol 4, 177-197

4. Leonardi, R., Zhang, Y. M., Rock, C. O., and Jackowski, S. (2005) Coenzyme A: back in action. Progress in lipid research 44, 125-153

5. Choudhary, C., Weinert, B. T., Nishida, Y., Verdin, E., and Mann, M. (2014) The growing landscape of lysine acetylation links metabolism and cell signalling. Nature reviews. Molecular cell biology 15, 536-550

6. Hirschey, M. D., and Zhao, Y. (2015) Metabolic Regulation by Lysine Malonylation, Succinylation, and Glutarylation. Molecular \& cellular proteomics : MCP 14, 2308-2315

7. Resh, M. D. (2016) Fatty acylation of proteins: The long and the short of it. Progress in lipid research 63, 120-131

8. Daniotti, J. L., Pedro, M. P., and Valdez Taubas, J. (2017) The role of S-acylation in protein trafficking. Traffic

9. Sabari, B. R., Zhang, D., Allis, C. D., and Zhao, Y. (2017) Metabolic regulation of gene expression through histone acylations. Nature reviews. Molecular cell biology 18, 90-101

10. Horie, S., Ishii, H., and Suga, T. (1981) Changes in peroxisomal fatty acid oxidation in the diabetic rat liver. Journal of biochemistry 90, 1691-1696

11. Van Broekhoven, A., Peeters, M. C., Debeer, L. J., and Mannaerts, G. P. (1981) Subcellular distribution of coenzyme A: evidence for a separate coenzyme A pool in peroxisomes. Biochemical and biophysical research communications 100, 305-312

12. Peng, Y., and Puglielli, L. (2016) N-lysine acetylation in the lumen of the endoplasmic reticulum: A way to regulate autophagy and maintain protein homeostasis in the secretory pathway. Autophagy 12, 1051-1052

13. Cooper, D. E., Young, P. A., Klett, E. L., and Coleman, R. A. (2015) Physiological Consequences of Compartmentalized Acyl-CoA Metabolism. The Journal of biological chemistry 290, 2002320031

14. Ferdinandusse, S., Denis, S., Faust, P. L., and Wanders, R. J. (2009) Bile acids: the role of peroxisomes. Journal of lipid research 50, 2139-2147

15. Leonardi, R., Rock, C. O., and Jackowski, S. (2014) Pank1 deletion in leptin-deficient mice reduces hyperglycaemia and hyperinsulinaemia and modifies global metabolism without affecting insulin resistance. Diabetologia 57, 1466-1475

16. Leonardi, R., Rehg, J. E., Rock, C. O., and Jackowski, S. (2010) Pantothenate kinase 1 is required to support the metabolic transition from the fed to the fasted state. PloS one 5, e11107

17. Reilly, S. J., Tillander, V., Ofman, R., Alexson, S. E., and Hunt, M. C. (2008) The nudix hydrolase 7 is an Acyl-CoA diphosphatase involved in regulating peroxisomal coenzyme A homeostasis. Journal of biochemistry 144, 655-663

18. Shumar, S. A., Kerr, E. W., Geldenhuys, W. J., Montgomery, G. E., Fagone, P., Thirawatananond, P., Saavedra, H., Gabelli, S. B., and Leonardi, R. (2018) Nudt19 is a renal CoA diphosphohydrolase with biochemical and regulatory properties that are distinct from the hepatic Nudt7 isoform. The Journal of biological chemistry

19. Zhang, Y. M., Rock, C. O., and Jackowski, S. (2006) Biochemical properties of human pantothenate kinase 2 isoforms and mutations linked to pantothenate kinase-associated neurodegeneration. The Journal of biological chemistry 281, 107-114 
20. Vallari, D. S., Jackowski, S., and Rock, C. O. (1987) Regulation of pantothenate kinase by coenzyme A and its thioesters. The Journal of biological chemistry 262, 2468-2471

21. Rock, C. O., Calder, R. B., Karim, M. A., and Jackowski, S. (2000) Pantothenate kinase regulation of the intracellular concentration of coenzyme A. The Journal of biological chemistry 275, 13771383

22. Ofman, R., Speijer, D., Leen, R., and Wanders, R. J. (2006) Proteomic analysis of mouse kidney peroxisomes: identification of RP2p as a peroxisomal nudix hydrolase with acyl-CoA diphosphatase activity. The Biochemical journal 393, 537-543

23. Gasmi, L., and McLennan, A. G. (2001) The mouse Nudt7 gene encodes a peroxisomal nudix hydrolase specific for coenzyme A and its derivatives. The Biochemical journal 357, 33-38

24. Shumar, S. A., Fagone, P., Alfonso-Pecchio, A., Gray, J. T., Rehg, J. E., Jackowski, S., and Leonardi, R. (2015) Induction of Neuron-Specific Degradation of Coenzyme A Models Pantothenate Kinase-Associated Neurodegeneration by Reducing Motor Coordination in Mice. PloS one 10, e0130013

25. Van Veldhoven, P. P. (2010) Biochemistry and genetics of inherited disorders of peroxisomal fatty acid metabolism. Journal of lipid research 51, 2863-2895

26. Wanders, R. J., Vreken, P., Ferdinandusse, S., Jansen, G. A., Waterham, H. R., van Roermund, C. W., and Van Grunsven, E. G. (2001) Peroxisomal fatty acid alpha- and beta-oxidation in humans: enzymology, peroxisomal metabolite transporters and peroxisomal diseases. Biochemical Society transactions 29, 250-267

27. Hunt, M. C., Tillander, V., and Alexson, S. E. (2014) Regulation of peroxisomal lipid metabolism: the role of acyl-CoA and coenzyme A metabolizing enzymes. Biochimie 98, 45-55

28. Fagone, P., Wright, J. F., Nathwani, A. C., Nienhuis, A. W., Davidoff, A. M., and Gray, J. T. (2012) Systemic errors in quantitative polymerase chain reaction titration of self-complementary adenoassociated viral vectors and improved alternative methods. Human gene therapy methods 23, 1-7

29. Garcia, M., Leonardi, R., Zhang, Y. M., Rehg, J. E., and Jackowski, S. (2012) Germline deletion of pantothenate kinases 1 and 2 reveals the key roles for CoA in postnatal metabolism. PloS one 7, e40871

30. Patro, R., Duggal, G., Love, M. I., Irizarry, R. A., and Kingsford, C. (2017) Salmon provides fast and bias-aware quantification of transcript expression. Nature methods 14, 417-419

31. Soneson, C., Love, M. I., and Robinson, M. D. (2015) Differential analyses for RNA-seq: transcript-level estimates improve gene-level inferences. F1000Res 4, 1521

32. Love, M. I., Huber, W., and Anders, S. (2014) Moderated estimation of fold change and dispersion for RNA-seq data with DESeq2. Genome Biol 15, 550

33. Bligh, E. G., and Dyer, W. J. (1959) A rapid method of total lipid extraction and purification. Can J Biochem Physiol 37, 911-917

34. Ivanova, P. T., Milne, S. B., Byrne, M. O., Xiang, Y., and Brown, H. A. (2007) Glycerophospholipid identification and quantitation by electrospray ionization mass spectrometry. Methods in enzymology 432, 21-57

35. Chong, J., Soufan, O., Li, C., Caraus, I., Li, S., Bourque, G., Wishart, D. S., and Xia, J. (2018) MetaboAnalyst 4.0: towards more transparent and integrative metabolomics analysis. Nucleic acids research 46, W486-W494

36. Minkler, P. E., Kerner, J., Ingalls, S. T., and Hoppel, C. L. (2008) Novel isolation procedure for short-, medium-, and long-chain acyl-coenzyme A esters from tissue. Analytical biochemistry 376, 275-276

37. Haynes, C. A. (2011) Analysis of mammalian fatty acyl-coenzyme A species by mass spectrometry and tandem mass spectrometry. Biochimica et biophysica acta 1811, 663-668

38. Magnes, C., Sinner, F. M., Regittnig, W., and Pieber, T. R. (2005) LC/MS/MS method for quantitative determination of long-chain fatty acyl-CoAs. Analytical chemistry 77, 2889-2894

39. Klaunig, J. E., Goldblatt, P. J., Hinton, D. E., Lipsky, M. M., Chacko, J., and Trump, B. F. (1981) Mouse liver cell culture. I. Hepatocyte isolation. In vitro 17, 913-925 
40. Zhang, W., Sargis, R. M., Volden, P. A., Carmean, C. M., Sun, X. J., and Brady, M. J. (2012) PCB 126 and other dioxin-like PCBs specifically suppress hepatic PEPCK expression via the aryl hydrocarbon receptor. PloS one 7, e37103

41. Cyphert, H. A., Alonge, K. M., Ippagunta, S. M., and Hillgartner, F. B. (2014) Glucagon stimulates hepatic FGF21 secretion through a PKA- and EPAC-dependent posttranscriptional mechanism. PloS one 9, e94996

42. Nathwani, A. C., Gray, J. T., Ng, C. Y., Zhou, J., Spence, Y., Waddington, S. N., Tuddenham, E. G., Kemball-Cook, G., McIntosh, J., Boon-Spijker, M., Mertens, K., and Davidoff, A. M. (2006) Self-complementary adeno-associated virus vectors containing a novel liver-specific human factor IX expression cassette enable highly efficient transduction of murine and nonhuman primate liver. Blood 107, 2653-2661

43. Otto, G. P., Rathkolb, B., Oestereicher, M. A., Lengger, C. J., Moerth, C., Micklich, K., Fuchs, H., Gailus-Durner, V., Wolf, E., and Hrabe de Angelis, M. (2016) Clinical Chemistry Reference Intervals for C57BL/6J, C57BL/6N, and C3HeB/FeJ Mice (Mus musculus). J Am Assoc Lab Anim Sci 55, 375-386

44. Russell, D. W. (2003) The enzymes, regulation, and genetics of bile acid synthesis. Annual review of biochemistry $\mathbf{7 2}, 137-174$

45. Li-Hawkins, J., Lund, E. G., Bronson, A. D., and Russell, D. W. (2000) Expression cloning of an oxysterol 7alpha-hydroxylase selective for 24-hydroxycholesterol. The Journal of biological chemistry 275, 16543-16549

46. Ramsay, R. R., and Zammit, V. A. (2004) Carnitine acyltransferases and their influence on CoA pools in health and disease. Molecular aspects of medicine 25, 475-493

47. Kivilompolo, M., Ohrnberg, L., Oresic, M., and Hyotylainen, T. (2013) Rapid quantitative analysis of carnitine and acylcarnitines by ultra-high performance-hydrophilic interaction liquid chromatography-tandem mass spectrometry. J Chromatogr A 1292, 189-194

48. Giesbertz, P., Ecker, J., Haag, A., Spanier, B., and Daniel, H. (2015) An LC-MS/MS method to quantify acylcarnitine species including isomeric and odd-numbered forms in plasma and tissues. Journal of lipid research 56, 2029-2039

49. $\quad$ van Vlies, N., Tian, L., Overmars, H., Bootsma, A. H., Kulik, W., Wanders, R. J., Wood, P. A., and Vaz, F. M. (2005) Characterization of carnitine and fatty acid metabolism in the long-chain acyl-CoA dehydrogenase-deficient mouse. The Biochemical journal 387, 185-193

50. Spiekerkoetter, U., Tokunaga, C., Wendel, U., Mayatepek, E., Ijlst, L., Vaz, F. M., van Vlies, N., Overmars, H., Duran, M., Wijburg, F. A., Wanders, R. J., and Strauss, A. W. (2005) Tissue carnitine homeostasis in very-long-chain acyl-CoA dehydrogenase-deficient mice. Pediatric research $57,760-764$

51. Mannaerts, G. P., and van Veldhoven, P. P. (1996) Functions and organization of peroxisomal betaoxidation. Annals of the New York Academy of Sciences 804, 99-115

52. Kasumov, T., Adams, J. E., Bian, F., David, F., Thomas, K. R., Jobbins, K. A., Minkler, P. E., Hoppel, C. L., and Brunengraber, H. (2005) Probing peroxisomal beta-oxidation and the labelling of acetyl-CoA proxies with [1-(13C)]octanoate and [3-(13C)]octanoate in the perfused rat liver. The Biochemical journal 389, 397-401

53. Arnauld, S., Fidaleo, M., Clemencet, M. C., Chevillard, G., Athias, A., Gresti, J., Wanders, R. J., Latruffe, N., Nicolas-Frances, V., and Mandard, S. (2009) Modulation of the hepatic fatty acid pool in peroxisomal 3-ketoacyl-CoA thiolase B-null mice exposed to the selective PPARalpha agonist Wy14,643. Biochimie 91, 1376-1386

54. Li, Y., Zalzala, M., Jadhav, K., Xu, Y., Kasumov, T., Yin, L., and Zhang, Y. (2016) Carboxylesterase 2 prevents liver steatosis by modulating lipolysis, endoplasmic reticulum stress, and lipogenesis and is regulated by hepatocyte nuclear factor 4 alpha in mice. Hepatology 63, 18601874

55. Okazaki, H., Igarashi, M., Nishi, M., Tajima, M., Sekiya, M., Okazaki, S., Yahagi, N., Ohashi, K., Tsukamoto, K., Amemiya-Kudo, M., Matsuzaka, T., Shimano, H., Yamada, N., Aoki, J., 
Morikawa, R., Takanezawa, Y., Arai, H., Nagai, R., Kadowaki, T., Osuga, J., and Ishibashi, S. (2006) Identification of a novel member of the carboxylesterase family that hydrolyzes triacylglycerol: a potential role in adipocyte lipolysis. Diabetes 55, 2091-2097

56. Quiroga, A. D., Li, L., Trotzmuller, M., Nelson, R., Proctor, S. D., Kofeler, H., and Lehner, R. (2012) Deficiency of carboxylesterase 1/esterase-x results in obesity, hepatic steatosis, and hyperlipidemia. Hepatology 56, 2188-2198

57. Blankman, J. L., Simon, G. M., and Cravatt, B. F. (2007) A comprehensive profile of brain enzymes that hydrolyze the endocannabinoid 2-arachidonoylglycerol. Chemistry \& biology 14, 1347-1356

58. Zhao, S., Mugabo, Y., Iglesias, J., Xie, L., Delghingaro-Augusto, V., Lussier, R., Peyot, M. L., Joly, E., Taib, B., Davis, M. A., Brown, J. M., Abousalham, A., Gaisano, H., Madiraju, S. R., and Prentki, M. (2014) alpha/beta-Hydrolase domain-6-accessible monoacylglycerol controls glucosestimulated insulin secretion. Cell metabolism 19, 993-1007

59. Thomas, G., Betters, J. L., Lord, C. C., Brown, A. L., Marshall, S., Ferguson, D., Sawyer, J., Davis, M. A., Melchior, J. T., Blume, L. C., Howlett, A. C., Ivanova, P. T., Milne, S. B., Myers, D. S., Mrak, I., Leber, V., Heier, C., Taschler, U., Blankman, J. L., Cravatt, B. F., Lee, R. G., Crooke, R. M., Graham, M. J., Zimmermann, R., Brown, H. A., and Brown, J. M. (2013) The serine hydrolase ABHD6 Is a critical regulator of the metabolic syndrome. Cell reports 5, 508-520

60. Horie, S., Isobe, M., and Suga, T. (1986) Changes in CoA pools in hepatic peroxisomes of the rat under various conditions. Journal of biochemistry 99, 1345-1352

61. Tokutake, Y., Iio, W., Onizawa, N., Ogata, Y., Kohari, D., Toyoda, A., and Chohnan, S. (2012) Effect of diet composition on coenzyme A and its thioester pools in various rat tissues. Biochemical and biophysical research communications 423, 781-784

62. Jin, Z., Bian, F., Tomcik, K., Kelleher, J. K., Zhang, G. F., and Brunengraber, H. (2015) Compartmentation of Metabolism of the C12-, C9-, and C5-n-dicarboxylates in Rat Liver, Investigated by Mass Isotopomer Analysis: ANAPLEROSIS FROM DODECANEDIOATE. The Journal of biological chemistry 290, 18671-18677

63. Bian, F., Kasumov, T., Thomas, K. R., Jobbins, K. A., David, F., Minkler, P. E., Hoppel, C. L., and Brunengraber, H. (2005) Peroxisomal and mitochondrial oxidation of fatty acids in the heart, assessed from the 13C labeling of malonyl-CoA and the acetyl moiety of citrate. The Journal of biological chemistry 280, 9265-9271

64. Suzuki, H., Yamada, J., Watanabe, T., and Suga, T. (1989) Compartmentation of dicarboxylic acid beta-oxidation in rat liver: importance of peroxisomes in the metabolism of dicarboxylic acids. Biochimica et biophysica acta 990, 25-30

65. Seedorf, U., Raabe, M., Ellinghaus, P., Kannenberg, F., Fobker, M., Engel, T., Denis, S., Wouters, F., Wirtz, K. W., Wanders, R. J., Maeda, N., and Assmann, G. (1998) Defective peroxisomal catabolism of branched fatty acyl coenzyme A in mice lacking the sterol carrier protein-2/sterol carrier protein-x gene function. Genes Dev 12, 1189-1201

66. Fan, C. Y., Pan, J., Usuda, N., Yeldandi, A. V., Rao, M. S., and Reddy, J. K. (1998) Steatohepatitis, spontaneous peroxisome proliferation and liver tumors in mice lacking peroxisomal fatty acyl-CoA oxidase. Implications for peroxisome proliferator-activated receptor alpha natural ligand metabolism. The Journal of biological chemistry 273, 15639-15645

67. Westin, M. A., Hunt, M. C., and Alexson, S. E. (2008) Short- and medium-chain carnitine acyltransferases and acyl-CoA thioesterases in mouse provide complementary systems for transport of beta-oxidation products out of peroxisomes. Cellular and molecular life sciences : CMLS 65, 982-990

68. Farrell, S. O., Fiol, C. J., Reddy, J. K., and Bieber, L. L. (1984) Properties of purified carnitine acyltransferases of mouse liver peroxisomes. The Journal of biological chemistry 259, 1308913095

69. Miyazawa, S., Ozasa, H., Osumi, T., and Hashimoto, T. (1983) Purification and properties of carnitine octanoyltransferase and carnitine palmitoyltransferase from rat liver. Journal of biochemistry 94, 529-542 
70. Westin, M. A., Alexson, S. E., and Hunt, M. C. (2004) Molecular cloning and characterization of two mouse peroxisome proliferator-activated receptor alpha (PPARalpha)-regulated peroxisomal acyl-CoA thioesterases. J Biol Chem 279, 21841-21848

71. Antonenkov, V. D., and Hiltunen, J. K. (2006) Peroxisomal membrane permeability and solute transfer. Biochimica et biophysica acta 1763, 1697-1706

72. Reszko, A. E., Kasumov, T., David, F., Jobbins, K. A., Thomas, K. R., Hoppel, C. L., Brunengraber, H., and Des Rosiers, C. (2004) Peroxisomal fatty acid oxidation is a substantial source of the acetyl moiety of malonyl-CoA in rat heart. The Journal of biological chemistry 279, 19574-19579

73. Taniguchi, M., Hayashi, T., Nii, M., Yamaguchi, T., Fujishima-Kanaya, N., Awata, T., and Mikawa, S. (2010) Fine mapping of quantitative trait loci for meat color on Sus scrofa chromosome 6: analysis of the swine NUDT7 gene. Journal of animal science 88, 23-31

74. Taniguchi, M., Hayashi, T., Nii, M., Yamaguchi, T., Fujishima-Kanaya, N., Awata, T., and Mikawa, S. (2010) Overexpression of NUDT7, a candidate quantitative trait locus for pork color, downregulates heme biosynthesis in L6 myoblasts. Meat science 86, 728-732

75. Labbe, R. F., Kurumada, T., and Onisawa, J. (1965) The role of succinyl-CoA synthetase in the control of heme biosynthesis. Biochimica et biophysica acta 111, 403-415

76. Chiang, J. Y. (2013) Bile acid metabolism and signaling. Compr Physiol 3, 1191-1212

77. O'Byrne, J., Hunt, M. C., Rai, D. K., Saeki, M., and Alexson, S. E. (2003) The human bile acidCoA:amino acid N-acyltransferase functions in the conjugation of fatty acids to glycine. The Journal of biological chemistry 278, 34237-34244

78. Pellicoro, A., van den Heuvel, F. A., Geuken, M., Moshage, H., Jansen, P. L., and Faber, K. N. (2007) Human and rat bile acid-CoA:amino acid N-acyltransferase are liver-specific peroxisomal enzymes: implications for intracellular bile salt transport. Hepatology 45, 340-348

79. Styles, N. A., Falany, J. L., Barnes, S., and Falany, C. N. (2007) Quantification and regulation of the subcellular distribution of bile acid coenzyme A:amino acid N-acyltransferase activity in rat liver. Journal of lipid research 48, 1305-1315

80. Hunt, M. C., Yang, Y. Z., Eggertsen, G., Carneheim, C. M., Gafvels, M., Einarsson, C., and Alexson, S. E. (2000) The peroxisome proliferator-activated receptor alpha (PPARalpha) regulates bile acid biosynthesis. The Journal of biological chemistry 275, 28947-28953

81. Shin, D. J., Campos, J. A., Gil, G., and Osborne, T. F. (2003) PGC-1alpha activates CYP7A1 and bile acid biosynthesis. The Journal of biological chemistry 278, 50047-50052

82. Song, J., Baek, I. J., Chun, C. H., and Jin, E. J. (2018) Dysregulation of the NUDT7-PGAM1 axis is responsible for chondrocyte death during osteoarthritis pathogenesis. Nature communications $\mathbf{9}$, 3427 


\section{$\underline{\text { Table and figure legends }}$}

Table 3.1. Selected features of fed and $24 \mathrm{~h}$ fasted GFP and Nudt7 mice. Values are expressed as the mean of 7-8 mice per condition \pm SEM. ${ }^{*} p \leq 0.05$ relative to GFP under the same nutritional conditions. \# $p \leq 0.05$, \#\# $p \leq 0.01$, and \#\#\# $p \leq 0.001$ relative to the fed state within GFP or Nudt7 groups. N/A, not applicable.

Table 3.2. Over-representation enrichment analysis of the genes differentially expressed between Nudt7 and GFP mice in the fasted state. The analysis was conducted using WebGestalt, as described in the 'Materials and methods' section.

Figure 3.1. Changes in hepatic CoA content, Nudt7 mRNA, and activity with fasting. Mice were fasted for 0-48 h and livers were harvested for the measurement of (A) total hepatic CoA levels, (B) Nudt7 mRNA, and (C) CoA-degrading activity using the Nudt7 substrate mBB-CoA, as described in 'Materials and methods'. Data are reported as the mean (horizontal line) of measurements on individual mice (circles) \pm SEM. \#\#, $p<0.01$, \#\#\#, $p<0.001$ relative to the fed state at $\mathrm{t}=0$.

Figure 3.2. Liver-specific overexpression of Nudt7 and GFP and effect on hepatic CoA levels. C57BL/6J mice were injected with AAV particles to achieve liver-specific overexpression of FLAG-tagged Nudt7 or the control protein GFP. (A) Western blot analysis of liver homogenates (5 $\mu \mathrm{g}$ of protein) for GFP expression at different time points, following the intravenous injection of AAV particles. (B) Three weeks after GFP-AAV injection, western blot analysis of different tissue homogenates (5 $\mu \mathrm{g}$ of protein) confirmed that transgene expression was liver-specific. (C) Liver homogenates obtained from non-injected mice or from mice injected with Nudt7- and GFP-AAV were assayed for total CoA-degrading activity. (C, inset) Western blot of liver homogenates (20 $\mu \mathrm{g}$ of protein) obtained from GFP and Nudt7 mice using the Nudt7 antibody. GAPDH was used as loading control in (A), (B), and (C, inset). Numbers in parenthesis represent the number of animals analyzed; data are reported as the mean \pm SEM. (D) Primary hepatocytes were isolated from Nudt7 mice, fixed and used to visualize the localization of the enzyme using the FLAG 
antibody and confocal microscopy. Nudt7 is shown in green and the PMP70 protein, which was used as a marker for the peroxisomes, is shown in red. Cell nuclei were stained with DAPI, shown in blue. The inset in the merged image shows details at a higher magnification. The results are representative of 2 independent experiments. (E) Total CoA measured in the liver of Nudt7 and GFP mice fed ad libitum or fasted for 24 and $48 \mathrm{~h}$. Data are reported as the mean (horizontal lines) of measurements on individual mice (circles and squares) \pm SEM. \#\#\#, $p<0.001$ relative to the fed state.

Figure 3.3. Liver acyl-CoA analysis. Livers isolated from (A-C) fed and (D-F) $24 \mathrm{~h}$ fasted Nudt7 and GFP mice were analyzed by LC/MS/MS to determine the relative abundance of (A, D) free and acetylCoA, (B, E) short-chain acyl-CoAs and (C, F) medium- and long-chain acyl-CoAs. Numbers in parenthesis represent the number of animals analyzed; data are reported as the mean \pm SEM. ${ }^{*}, p<0.05$.

Figure 3.4. Global metabolic profiling of $24 \mathrm{~h}$ fasted Nudt7 and GFP livers. (A) Number of metabolites significantly changed in the Nudt7 and GFP livers by fasting and, within a given nutritional state, by Nudt7 overexpression. Under each condition, the numbers in red represent metabolites whose concentrations increased and the numbers in blue represent metabolites whose concentrations decreased. (B) Score plot of the principal-component analysis (PCA) obtained using MetaboAnalyst 4.0. Colored ellipses represent 95\% confidence intervals for each group. Colored dots represent individual mice. (C) Top 3 most significantly enriched pathways that emerged from the global metabolic profiling.

Figure 3.5. Nudt7 overexpression in the fasted state decreases the hepatic bile acid content. (A) Heat plot of all primary and secondary bile acids, plus the bile acid precursors cholesterol and $7 \alpha$ hydroxycholesterol, detected by global metabolic profiling. Targeted bile acid analysis of (B) livers, (C) gall bladder extracts, and (D) small intestine. In (B-D), data are reported as the mean \pm SEM and numbers in parenthesis represent the number of animals analyzed. ${ }^{*}, p<0.05$. (E) Scheme of the conversion of DHCA-CoA and THCA-CoA to chenodeoxycholic acid (CDCA) and cholic acid derivatives in the peroxisomes. Known substrates of Nudt7 are marked by an asterisk. Overexpression of Nudt7 was associated with a decrease (thick blue arrows) in the concentration of choloyl-CoA (CA-CoA), propionyl- 
CoA (Prop-CoA), and of several primary and secondary bile acids, as detected by either untargeted or targeted bile acid analyses. The decrease in free CoA, which narrowly missed statistical significance ( $p=0.07$ ), is indicated by the thin dashed arrow. CA, cholic acid; CAS, cholic acid sulfate; CDCA, chenodeoxycholic acid; DCA, deoxycholic acid; 7keto-DCA, 7-keto-deoxycholic acid; HDCA, hyodeoxycholic acid; 3DHCA, 3-dehydrocholic acid; 6OH-LCA, 6-hydroxy-lithocholic acid; 6oxo-LCA, 6-oxo-lithocholic acid; G-CA, glycocholic acid; G- $\beta$ MCA, glyco- $\beta$-muricholic acid; HCA, hyocholic acid; HDCA, hyodeoxycholic acid; LCA, lithocholic acid; $\alpha$ MCA, $\alpha$-muricholic acid; $\beta$ MCA, $\beta$-muricholic acid;

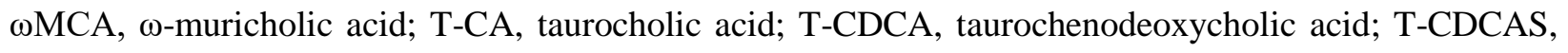
taurochenodeoxycholic acid sulfate; T-DCA, taurodeoxycholic acid; T- $\alpha$ MCA, tauro- $\alpha$-muricholic acid; T$\beta$ MCA, tauro- $\beta$-muricholic acid; T-LCA, taurolithocholic acid; T-HDCA, taurohyodeoxycholic acid; TUDCA, tauroursodeoxycholic acid; UDCA, ursodeoxycholic acid.

Figure 3.6. Nudt7 overexpression in the fasted state decreases the rate of peroxisomal fatty acid oxidation. (A) Heat plot of all the short-, medium-, and long-chain acylcarnitines, plus free carnitine and deoxycarnitine, detected by global metabolic profiling. (B) Primary hepatocytes were isolated from fasted Nudt7 and GFP mice and incubated with $\left[1-{ }^{14} \mathrm{C}\right]$-palmitic acid in the presence or absence of etomoxir, as described in 'Materials and methods'. Data are reported as the mean (horizontal bars) of measurements on individual mice (circles and squares) \pm SEM. ${ }^{*}, p<0.05$. (C) Schematic representation of the oxidation of medium- and long-chain acyl-CoAs (C-20-acyl-CoAs) to short-chain acyl-CoAs in the peroxisomes. Known substrates of Nudt7 are marked by an asterisk. The half-filled circle indicates that some $\mathrm{C}_{8-20}$-acylCoAs (i.e. octanoyl- and lauroyl-CoA) are good substrate of Nudt7, but some others (i.e. stearoyl-CoA) are not. A decrease in the rate of peroxisomal fatty acid oxidation in the Nudt7 livers correlated with a decrease in the concentration of short-chain acyl-CoAs and correspondent carnitines (thick blue arrows), together with the accumulation of $\mathrm{C}_{8-20}$-acylcarnitines. The decrease in free CoA missed statistical significance $(p=0.07)$ and this is indicated the thin dashed arrow. Acylcarnitines derived from peroxisomal acyl-CoAs can be formed directly in the peroxisomes or outside the organelles, following the release of the acyl groups 
as free fatty acids. Unlike acyl-CoAs, which are too bulky, the acylcarnitines formed inside the peroxisomes can leave the organelles (71). 
$\underline{\text { Tables and figures }}$

Table 3.1. Selected features of fed and $24 \mathrm{~h}$ fasted GFP and Nudt7 mice.

\begin{tabular}{|c|c|c|c|c|}
\hline & \multicolumn{2}{|c|}{ Fed } & \multicolumn{2}{|c|}{24 h Fast } \\
\hline & GFP & Nudt7 & $G F P$ & Nudt7 \\
\hline Serum ALT (mg/dL) & $21 \pm 2$ & $18 \pm 1$ & $35 \pm 4 \#$ & $24 \pm 3$ \\
\hline Serum AST (mg/dL) & $48 \pm 6$ & $36 \pm 7$ & $67 \pm 9$ & $62 \pm 9 \#$ \\
\hline Blood glucose (mg/dL) & $98 \pm 6$ & $105 \pm 6$ & $61 \pm 3$ \#\#\# & $55 \pm 3 \# \# \#$ \\
\hline $\begin{array}{l}\text { Serum 3-hydroxybutyrate } \\
\text { (mM) }\end{array}$ & $0.31 \pm 0.04$ & $0.38 \pm 0.08$ & $1.63 \pm 0.15$ \#\#\# & $1.57 \pm 0.08$ \#\#\# \\
\hline Serum triglycerides $(\mathrm{mg} / \mathrm{dL})$ & $119 \pm 18$ & $144 \pm 10$ & $86 \pm 6$ & $83 \pm 4$ \#\#\# \\
\hline Serum cholesterol $(\mathrm{mg} / \mathrm{dL})$ & $106 \pm 8$ & $90 \pm 6$ & $67 \pm 6 \# \#$ & $68 \pm 3 \# \#$ \\
\hline Food intake (g/mouse/day) & $4.4 \pm 0.5$ & $4.9 \pm 0.2$ & N/A & N/A \\
\hline Body weight (g) & $30.0 \pm 0.5$ & $30.8 \pm 0.2$ & $24.2 \pm 0.3$ \#\#\# & $24.3 \pm 0.6 \# \# \#$ \\
\hline $\begin{array}{l}\text { Liver weight/body weight } \\
(\mathrm{mg} / \mathrm{g})\end{array}$ & $44.6 \pm 1.9$ & $50.3 \pm 1.7 *$ & $35.5 \pm 1.4$ \#\# & $39.4 \pm 1.4$ \#\#\# \\
\hline Liver triglycerides $(\mathrm{mg} / \mathrm{g})$ & $0.9 \pm 0.2$ & $0.9 \pm 0.1$ & $19.4 \pm 1.9$ \#\#\# & $24.1 \pm 6.0$ \#\# \\
\hline Liver cholesterol (mg/g) & $0.6 \pm 0.1$ & $0.5 \pm 0.1$ & $1.1 \pm 0.1 \# \# \#$ & $0.9 \pm 0.10 \# \#$ \\
\hline
\end{tabular}


Table 3.2. Over-representation enrichment analysis of the genes differentially expressed between Nudt7 and GFP mice in the fasted state.

Molecular function: carboxylic ester hydrolase activity (term: GO: 000052689)

Fold enrichment: 10.7, $p=0.0000171$, FDR $=0.004$

\begin{tabular}{ccc}
\hline Gene symbol & Gene name & Fold change, Nudt7/GFP \\
\hline Car2 & Carbonic anhydrase 2 & 2.11 \\
Ces1e & Carboxylesterase 1E & 1.61 \\
Ces1f & Carboxylesterase 1F & 1.98 \\
Ces1g & Carboxylesterase 1G & 2.11 \\
Ces2c & Carboxylesterase 2C & 3.17 \\
Abhd6 & Abhydrolase domain containing 6 & 1.81 \\
\hline
\end{tabular}

Molecular function: fibronectin binding (term: GO: 0001968)

Fold enrichment: 29.4, $p=0.00013, F D R=0.015$

\begin{tabular}{ccc}
\hline Gene symbol & Gene name & Fold change, Nudt7/GFP \\
\hline Itgb3 & Integrin beta 3 & 0.48 \\
Sdc4 & Syndecan 4 & 0.66 \\
Lrrc15 & Leucine rich repeat containing 15 & 0.48 \\
\hline
\end{tabular}

Pathway: PPAR signaling pathway (ID: WP2316)

Fold enrichment: 9.4, $p=0.00015, \mathrm{FDR}=0.025$ 


\begin{tabular}{ccc}
\hline Gene symbol & Gene name & Fold change, Nudt7/GFP \\
\hline Cd36 & CD36 antigen & 2.32 \\
Cyp4a12b & Cytochrome P450, family 4, subfamily a, & 1.96 \\
Pltp & polypeptide 12B & 1.55 \\
Scd1 & Stearoyl-Coenzyme A desaturase 1 & 2.20 \\
Acaa1b & Acetyl-Coenzyme A acyltransferase 1B & 2.02 \\
\hline
\end{tabular}

\section{Supplementary Table 3.S1. Global metabolic profiling}

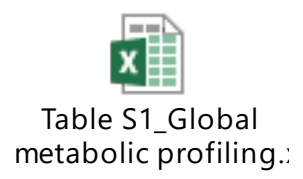

Supplementary Table 3.S2. Acyl-CoA LC/MS/MS parameters

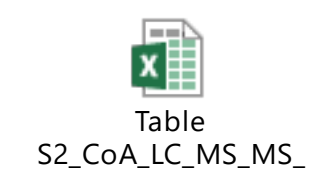

\section{Supplementary Table 3.S3. Nudt7-AAV RNAseq results}

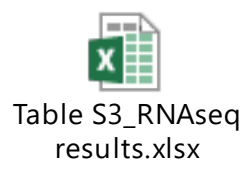



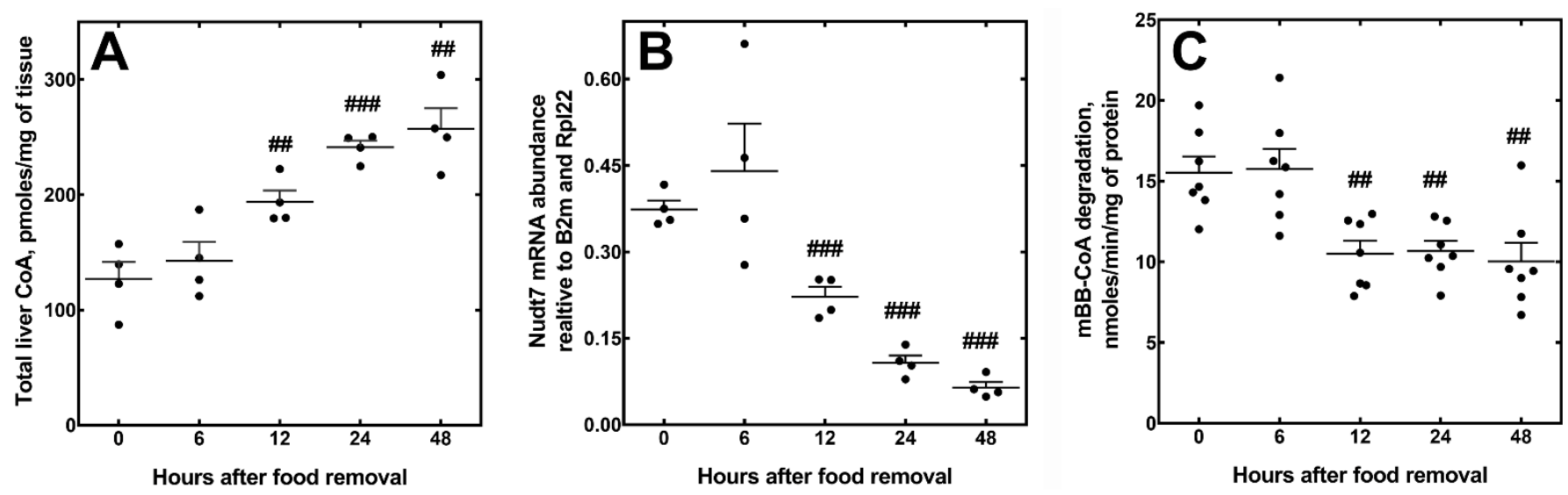

Figure 3.1. Changes in hepatic CoA content, Nudt7 mRNA, and activity with fasting. 

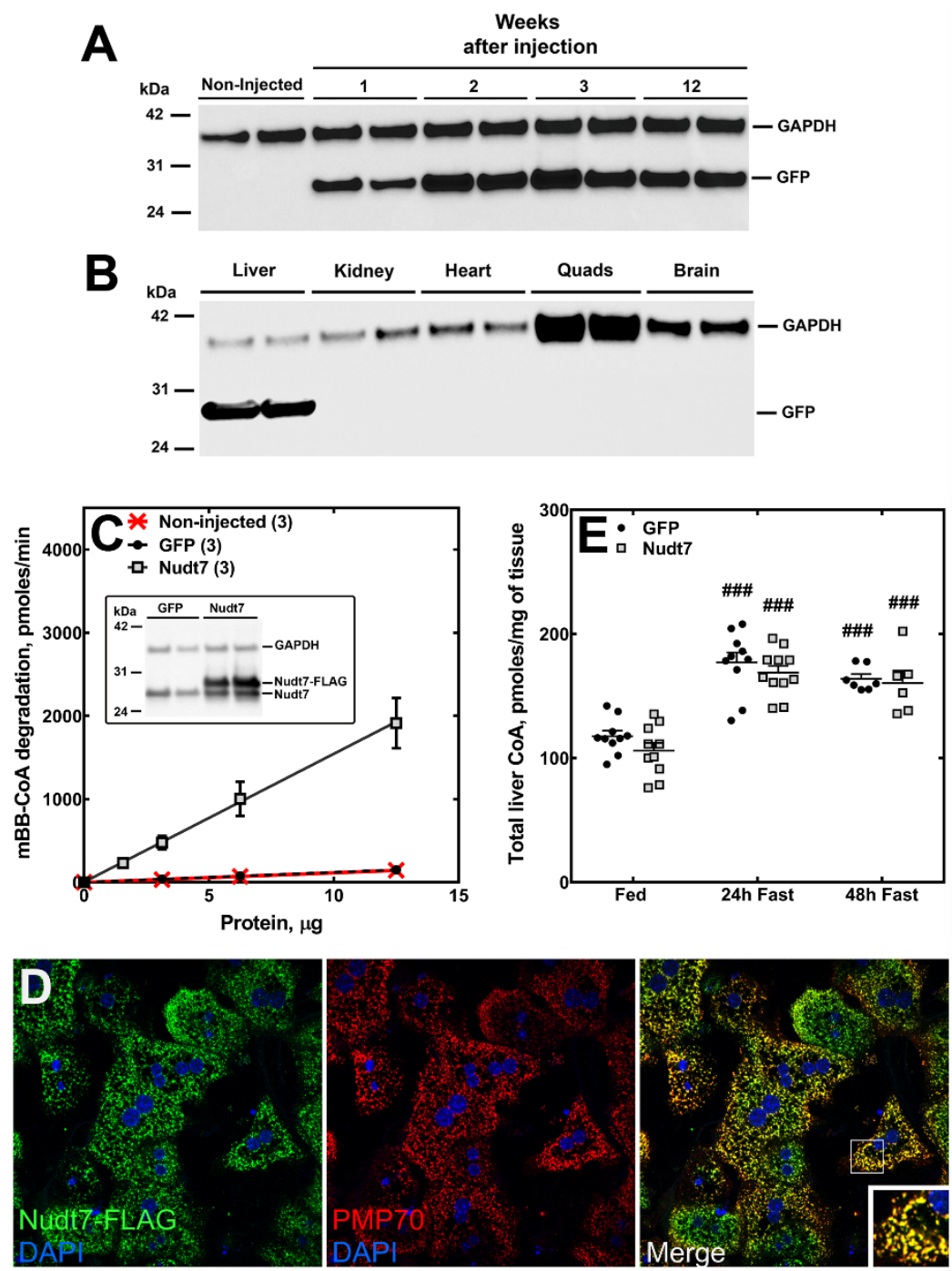

Figure 3.2. Liver-specific overexpression of Nudt7 and GFP and effect on hepatic CoA levels. 

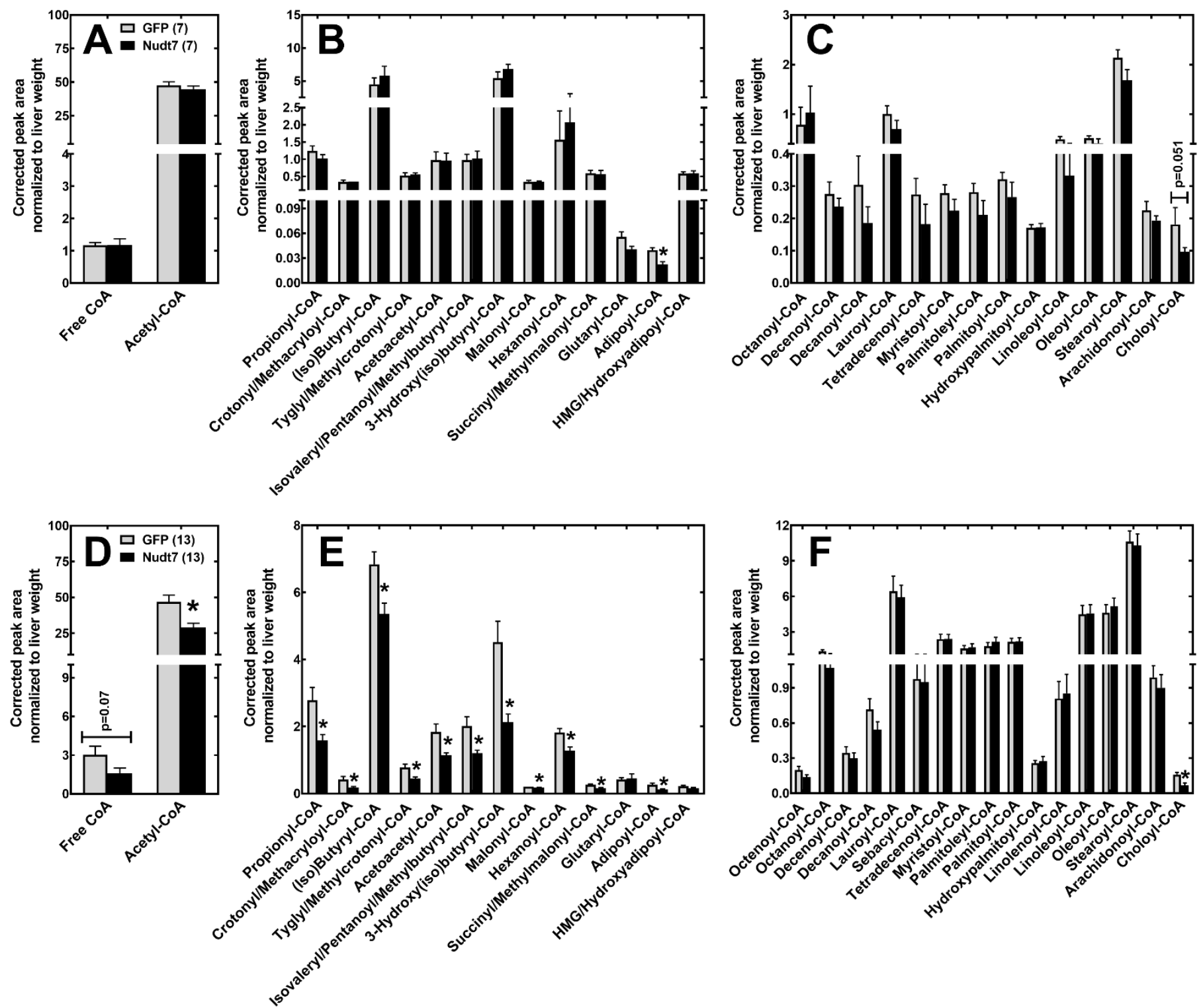

Figure 3.3. Liver acyl-CoA analysis. 


\begin{tabular}{cccccc}
\hline A & $\begin{array}{c}\text { Metabolites } \\
\text { changed } \\
\text { Metabolites } \\
\text { detected= }\end{array}$ & \multicolumn{2}{c}{ Fasted/Fed } & Fed & Fasted \\
\cline { 2 - 7 } 769 & To.05 & GFP & Nudt7 & Nudt7/GFP & Nudt7/GFP \\
\cline { 2 - 7 } & $\uparrow$ & 479 & 465 & 66 & 111 \\
& $\downarrow$ & 301 & 257 & 29 & 50 \\
& 178 & 208 & 37 & 61 \\
\hline
\end{tabular}
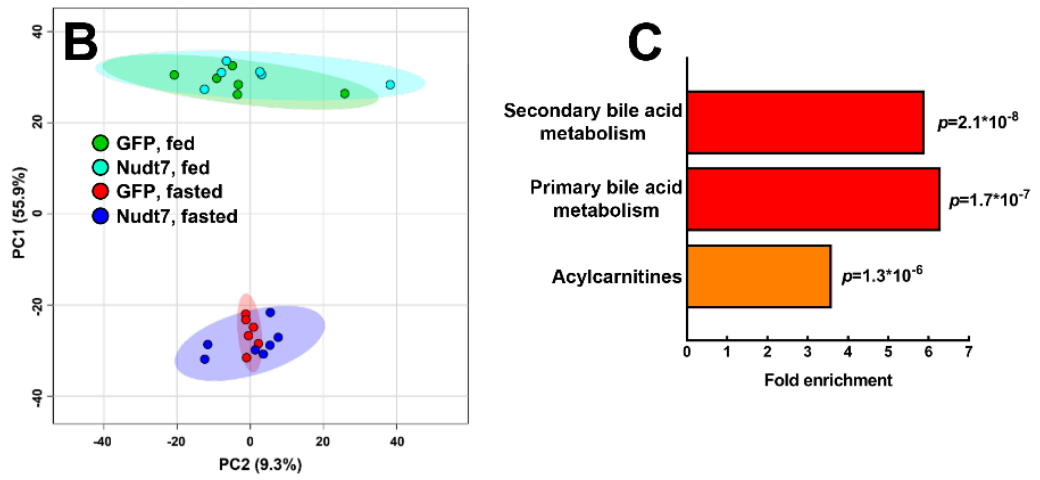

Figure 3.4. Global metabolic profiling of $24 \mathrm{~h}$ fasted Nudt7 and GFP livers. 

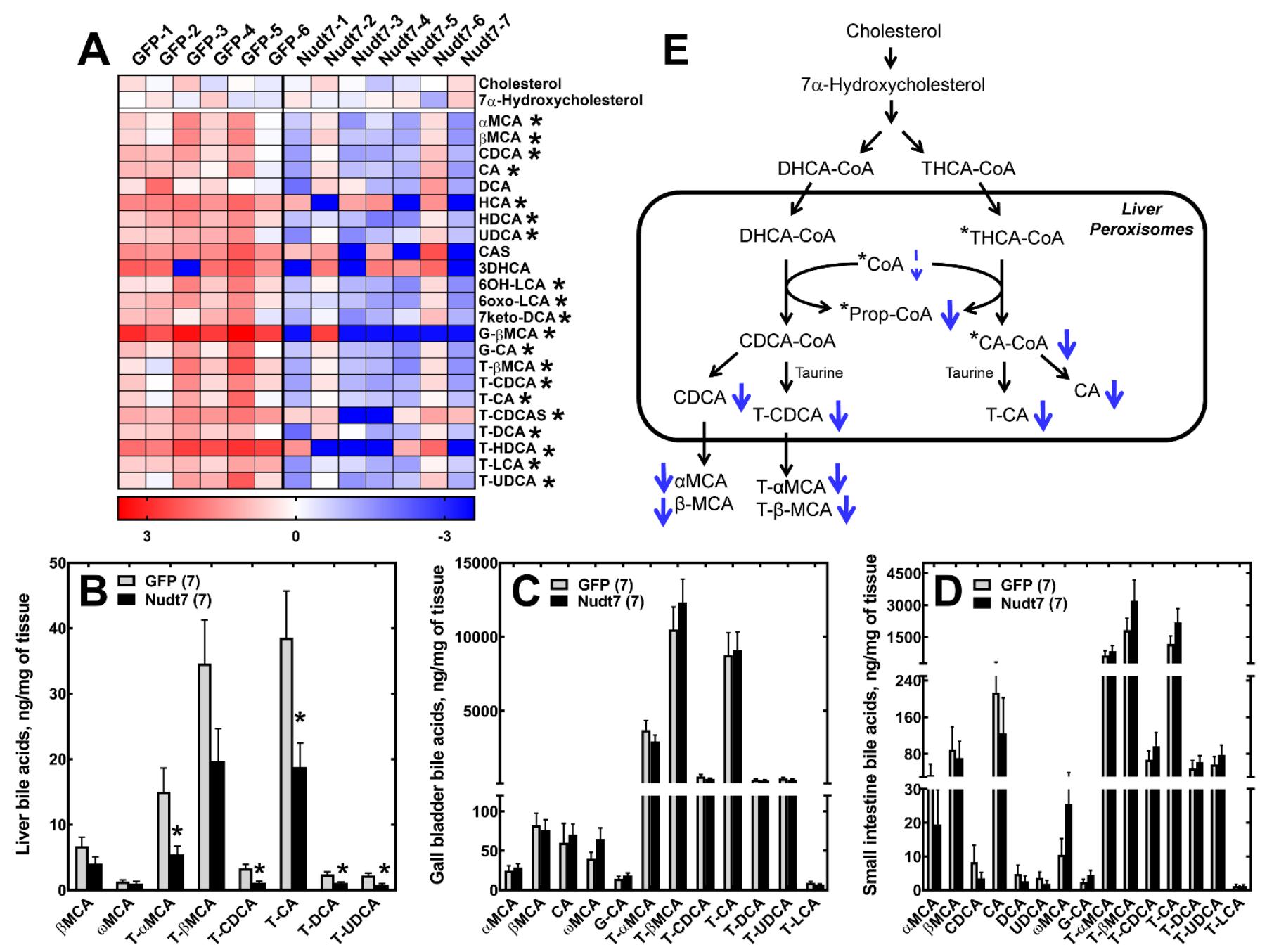

Figure 3.5. Nudt7 overexpression in the fasted state decreases the hepatic bile acid content. 
A
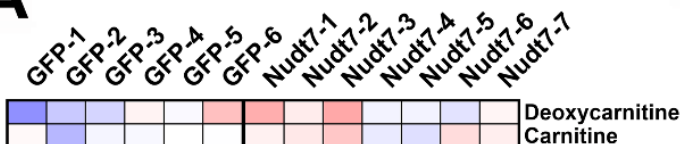

Acetylcarnitine, $\mathrm{C2}$

Propionylcarnitine, C3 *

Malonylcarnitine, C3-DC

Isobutyrylcarnitine, $\mathrm{C} 4$

3-Hydroxybutyrylcarnitine, $\mathrm{C} 4-\mathrm{OH}$

Succinylcarnitine, C4-DC *

Isovalerylcarnitine, C5

2-Methylbutyrylcarnitine, C5

Glutarylcarnitine, C5-DC *

Tiglylcarnitine, C5:1-DC

Adipoylcarnitine, C6-DC *

Pimeloyl/3-Methyladipoylcarnitine, C7-DC

Suberoylcarnitine, C8-DC *

cis-4-Decenoylcarnitine, C10:1 *

Lauroylcarnitine, $\mathrm{C} 12$ *

5-Dodecenoylcarnitine, C12:1*

Myristoylcarnitine, C14 *

Myristoleoylcarnitine, C14:1*

Pentadecanoylcarnitine, C15 *

Palmitoylcarnitine, C16 *

Palmitoleoylcarnitine, C16:1 *

Margaroylcarnitine, $\mathrm{C} 17$

Stearoylcarnitine, C18 *

Oleoylcarnitine, C18:1*

Linoleoylcarnitine, C18:2 *

Arachidoylcarnitine, C20 *

icosenoylcarnitine, C20:1 $* 2$

Dihomo-Linolenoylcarnitine, 20:3

Arachidonoylcarnitine, C20:4

Arachidonoylcarnitine,

Behenoylcarnitine, C22

Docosahexaenoylcarnitine, C22:6

Lignoceroylcarnitine, C24

Nervonoylcarnitine, C24:1

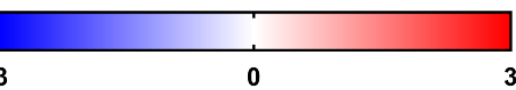

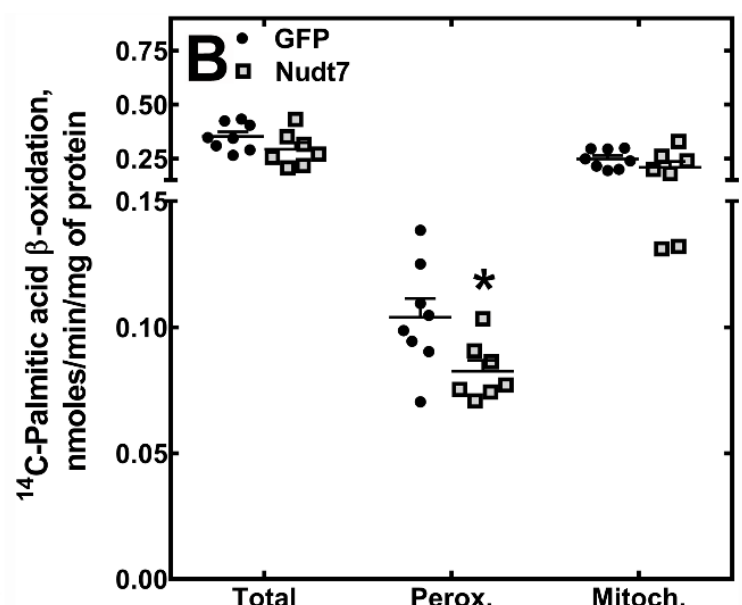

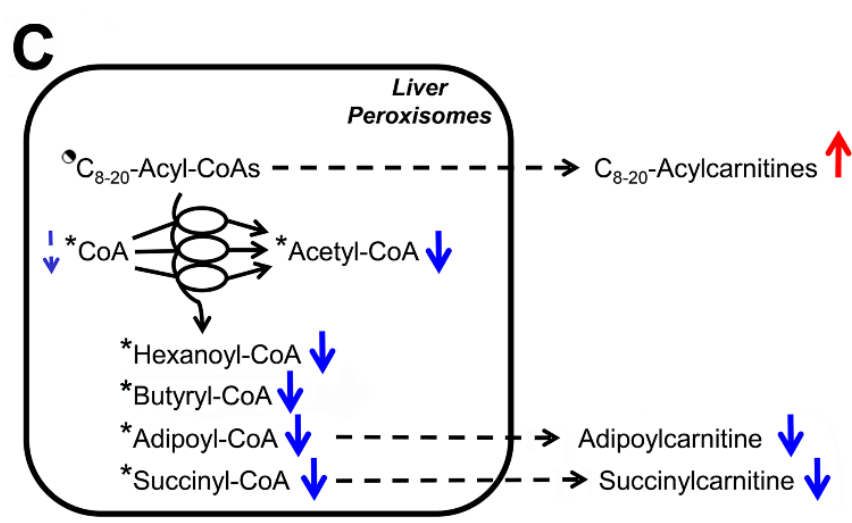

Figure 3.6. Nudt7 overexpression in the fasted state decreases the rate of peroxisomal fatty acid oxidation. 


\section{Chapter IV: Conclusions and future directions}

A multitude of research has been dedicated to and has confirmed that CoA is essential. Loss of CoA synthesis leads to drastic, negative consequence due to reduced flux through key metabolic pathways, disruption of gene regulation, and loss of protein regulation. Switching between the fed and fasted state requires dynamic adjustment of CoA synthesis. Synthesis of CoA in the liver increases in the fasted state to support the switch from glucose oxidation to fatty acid oxidation and gluconeogenesis to continue to provide energy for the body (1-3). Upon synthesis, CoA is distributed among the cytosol/nucleus, mitochondria, and peroxisomes to participate in the metabolic pathways in these subcellular compartments. It has remained unclear if the CoA pool in each compartment is regulated independently of CoA biosynthesis in the cytosol to modulate internal metabolic pathways. In the last decade, two CoA degrading enzymes were discovered in mammals. These enzymes were determined to be members of the Nudix hydrolase super-family, specific for only CoA, and to localize to the peroxisomes (4-7). Nudt7 and Nudt19 are expressed almost exclusively in the liver and kidneys, respectively, two very metabolically active organs. The objectives of this thesis were to use in vitro and in vivo biochemical approaches to: 1) determine the structural and biochemical properties of Nudt7 and Nudt19 and 2) identify the physiological role that Nudt7 plays in liver CoA regulation and metabolism.

\section{Conclusions of chapter II}

We first focused on the biochemical characterization of Nudt7 and Nudt19 using enzymatic assays, mutagenesis, and molecular modeling to characterize both proteins. Two key findings of this approach were the identification of chenodeoxycholic acid (CDCA) as a competitive inhibitor of Nudt19 but not Nudt7 and the determination that the CoA box and Nudix box motifs of the active sites were not interchangeable between the two enzymes. These findings led to the conclusion that even though both enzymes catalyze the same reaction, Nudt7 and Nudt19 exhibit distinct regulatory properties and likely possess different CoAbinding site and may be used for different purposes in the liver and kidneys, respectively. For example, bile 
acid synthesis and $\beta$-oxidation of very long chain fatty acids occur in the liver peroxisomes. As we have shown through our in vivo studies, Nudt7 may be present in the peroxisomes for the localized regulation of CoA levels to increase or decrease the flux through theses pathway depending on the nutritional state. Nudt19 may assist in regulating $\beta$-oxidation or other peroxisomal metabolic processes in the kidneys.

\section{Future directions of chapter II}

While molecular modeling was a useful resource to get an idea of the structure of Nudt19, it is not without limitations. There is currently no crystal structure for Nudt19 and the crystal structure of the human NUDT7 contains no bound substrate (8). Thus, our modeling of the Nudt19 structure may not be fully accurate. While our mutagenesis studies are consistent with the computational models that were generated, there will likely be inaccuracies about the exact positioning of the free CoA substrate its interactions with amino acids that we did not test. Solving the crystal structure of Nudt19 and generating crystal structures of both enzymes with substrate will be key to determining exact differences in the active sites and substrate binding. This approach may also be useful in determining if Nudt19 has any other domains that can interact with other proteins or metabolites for regulation.

Unlike Nudt7, Nudt19 levels do not change between nutritional states and it is still unknown if there is a post-translational mechanism that can regulate its activity. One possible way that the activity of Nudt19 and its involvement in peroxisomal metabolism may be through regulation of import into the peroxisomes. Import of peroxisomal matrix proteins is a fairly complex process as these proteins are synthesized in the cytosol and then imported into the peroxisomes in a fully folded confirmation (9). Many peroxisomal proteins, including Nudt7 and Nudt19, contain a C-terminal PTS1 sequence that binds to peroxisomal biogenesis factor 5 (Pex5) initiating the formation of a pore in the peroxisomal membrane and import of the protein into the lumen of the organelle (10). There are still many poorly understood steps in this process. Recently, it has been discovered that ubiquitination plays a role in stabilization of Pex5 for protein import and deubiquitination allows Pex5 to be recycled back to the cytosol (11-13). This mechanism may aid regulating amount of Nudt19 translocated to peroxisomes depending on the requirement of CoA 
in this organelle under different nutritional conditions. Another possible way that Nudt19 may be regulated is through CoAlation. CoAlation is a recently discovered covalent attachment of CoA to cysteine residues of proteins $(14,15)$. This type of modification has been discovered on a variety of different proteins under various conditions, modifies the activity of these proteins, and is reversible. Nudt19 contains nine cysteine residues so it is possible that one of these residues could be modified by CoA. Isolation of peroxisomes and cytosol from fed and fasted kidneys and analysis via Western blot may be one way of determining if Nudt19 is regulated through either of these mechanisms. Western blot analysis of the various subcellular compartments could show changes in the amount of Nudt19 in different subcellular compartments in the different nutritional states indicating that Nudt19 peroxisomal import is regulated. We can also attempt to immunoprecipitate Nudt19 from the peroxisomes and blot with antibodies against ubiquitin and CoA to see if the protein itself is post-translationally modified by either of these.

\section{Conclusions of chapter III}

Next, we began to elucidate the physiological role of Nudt7 by overexpressing Nudt7 in the liver. While overexpression of Nudt7 did not affect the total CoA pool in the liver, it did significantly decrease select short chain acyl-CoAs and choloyl-CoA, a CoA species formed only in the peroxisomes. These results are consistent with substrate specificity of Nudt7 in vitro. Furthermore, the increase in acyl-CoA species correlated with a decrease in two peroxisomal pathways that rely heavily on free CoA, $\beta$-oxidation

and bile acid synthesis. Finally, these changes in acyl-CoA levels and metabolic pathways were only observed in the fasted state when endogenous Nudt7 levels decrease significantly. Our findings suggest that Nudt7 activity is important for regulating select acyl-CoA species in the liver and the decrease in CoA degrading activity may be required for the increase in peroxisomal $\beta$-oxidation and bile acid synthesis during the fed to fasted transition.

\section{Future directions of chapter III}

While we now have initial evidence on the involvement of Nudt7 in liver metabolism, the next steps will be to confirm these findings in our $N u d t 7^{-/-}$mouse model. We already detect a similar trend in 
these mice, in that there is no change in total hepatic CoA levels, but we detect an increase in short chain acyl-CoA species that were decreased by Nudt7 over-expression (unpublished data). Our published data point to regulation of the peroxisomal CoA pool only; however, confirmation of this hypothesis will require analysis of the size and composition of the CoA pools in the subcellular compartments between wild type and $N u d t 7^{-/}$mice. This analysis will allow us to see if the changes observed at the whole tissue level are due to changes in the composition of the peroxisomal CoA pool. As of now, it is technically very difficult to isolate intact peroxisomes that are not contaminated by mitochondria while still isolating enough of the organelles to detect acyl-CoAs in this CoA pool. Peroxisomes contain the smallest pool of CoA (about 4$10 \%)$, which makes it a challenge to detect individual CoA species in this compartment. Future method development will be essential for getting a better perspective of changes in metabolites, especially CoA species, within this subcellular compartment. The best approach would be to use an immunoprecipitation procedure such as that developed by Chen et. Al (16). Unlike typical subcellular fractionation procedures, this technique is much faster, yields more material, and is more specific. Almost all organelle isolation methods take several hours resulting in loss of material and changes in metabolite composition (17). This is particularly troublesome for peroxisomes, which are extremely fragile and have been found to open and close during long isolation procedures resulting in the isolation of peroxisomal ghosts (18). Also, even under the most stringent conditions, there is still contamination of the "purified" organelles from other subcellular compartments. Typical fractionation procedures also require buffers that are generally incompatible with downstream applications such as mass spectrometry. Developing an immunoprecipitation procedure specific for peroxisomes would allow us to overcome these technical difficulties and analyze the changes in metabolites within these organelles. Nevertheless, since the previously developed immunoprecipitation procedure has been optimized for mitochondria, the conditions of the homogenization and immunoprecipitation itself may still need further optimization to support isolation of the peroxisomes. The same problem may still arise in a peroxisomal-specific immunoprecipitation with the isolation of empty peroxisomes due to their ability to open and reclose. 
Finally, in order to gain a broader perspective on the requirement of Nudt7 in the liver and its involvement in metabolic diseases, it will be important to use a nutritional mechanism in the $\mathrm{Nudt}^{-/ /}$mice to challenge the peroxisomal metabolic pathways and determine any adverse effects (or benefits) that may result from less Nudt7 activity in the liver. Nutritional studies in mice that mimic the Western (or fast food) diet use a combination of high fat and high cholesterol $(19,20)$. Both would challenge the peroxisomal pathways regulated by Nudt7; high fat would challenge the $\beta$-oxidation pathway and cholesterol would challenge the bile acid synthesis. Lack of Nudt7 may have differing effects for challenging these two pathways. Less Nudt7 in the peroxisomes would lead to increased free CoA in the Nudt $7^{-/}$mice, increasing peroxisomal $\beta$-oxidation and decreasing triglyceride accumulation in the liver. Therefore, these mice may be protected from the effects of the high fat in the Western diet. Alternatively, less Nudt7 could increase bile acid synthesis leading to an increase in cholesterol absorption from the diet.

The discovery that Nudt7 was involved in regulating bile acid synthesis was novel and surprising. As with most other metabolic pathways, bile acid synthesis is tightly regulated through transcriptional regulation of cholesterol 7alpha-hydroxylase (Cyp7a), the rate limiting enzyme of bile acid synthesis in the liver. The results of Chapter III show that regulation of a metabolite for various enzymes that require it also limit the flux through the metabolic pathway. The limitation to this overexpression study was that Nudt7 was overexpressed in adult mice, long after the enterohepatic bile acid pool was established. Once established, it is very difficult to affect the size of the total bile acid pool due to efficient reabsorption of bile acids in the small intestine (21). It will be interesting to analyze the composition and size of the bile acid pool in the $\mathrm{Nudt}^{-/}$mice to observe how CoA degradation can affect the bile acid synthesis during the establishment of this pool. Based on the current results, it is highly likely that a decrease in CoA degradation will enhance the size of the peroxisomal CoA pool and therefore increase bile acid synthesis and the size of the total bile acid pool. While this type of change may not lead to detectable whole-body changes under “normal conditions”, it may lead to alterations under nutritional challenges such as a Western diet. Future studies such as this may establish Nudt7 and the availability of CoA as a second key regulatory mechanism in bile acid synthesis. 
One of the most widely accepted methods of metabolic regulation is through transcriptional regulation of various enzymes involved in the numerous metabolic pathways (22). Recent studies, such as the one discussed in Chapter III, are beginning to show that metabolic intermediates and cofactors also play an important role in regulating metabolic pathways. Previous studies have shown that levels of cofactors such as CoA can affect cellular processes such as gluconeogenesis. To our knowledge, this study is the first to show that degradation of CoA can play a pivotal role in regulating CoA in one subcellular compartment independently of other compartments and that this regulation can lead to changes in organelle specific metabolic pathways. However, there are still many unknowns about CoA degradation and how it may affect other organs and metabolic pathways.

While we were able to establish that loss of Nudt19 in mice resulted in a significant increase in CoA in the fed state, we still observed a decrease in CoA upon refeeding that was also observed in the livers of mice lacking Nudt7 (unpublished data). The fact that accumulated CoA levels rapidly decrease upon refeeding even in the absence of the major CA degrading enzyme of that organ and lack of compensation by the other peroxisomal isoform indicates that there may be other yet undiscovered CoA degrading enzymes. For example, an uncharacterized Nudix hydrolase called Nudt8 has been annotated as a mitochondrial enzyme and is closely related to Nudt7 $(23,24)$. We have recently shown that Nudt8 is indeed a new CoA-degrading enzyme, which could play a key role in the regulation of the mitochondrial CoA pool (25). It will be useful in the future to generate Nudt7/Nudt19 double knockout mouse models as a better approach to discovering other CoA-degrading enzymes. These undiscovered enzymes may be responsible for the decrease in CoA levels observed in the transition from fasting to feeding in the absence of Nudt7 and Nudt19. Indeed, Nudt7 and Nudt19 are localized to the peroxisomes and based on our Nudt7 overexpression study are most likely important for regulating the peroxisomal CoA pool and peroxisomal processes. In addition to the mitochondria, there may additional CoA-degrading enzymes in the cytosol, or even the endoplasmic reticulum to also regulate their (acyl)-CoA pools independently of CoA biosynthesis.

One other future avenue of research that will be useful would be to study Nudt7, Nudt19, and other identified CoA degrading enzymes in the PanK1 $1^{--}$mice or HoPan treated mice. From studies in these two 
mouse models of impaired CoA biosynthesis, we know that CoA levels significantly decrease in the liver and kidney leading to detrimental effects on glucose metabolism. Knocking out Nudt7 in parallel with Pank1 or HoPan treatment could result in two possible results: 1) lack of Nudt7 could rescue the significant decrease in CoA and associated hypoglycemia in the liver, indicating that Nudt7 is the CoA degrading enzyme responsible for the metabolic changes observed in these mice and that the peroxisomal CoAdegradation can affect mitochondrial processes in certain situations or 2) these mice will maintain the same decrease in hepatic CoA and blood glucose indicating that a different enzyme may be another key player in regulating CoA levels. Further characterization of the known CoA-degrading enzymes and discovering other CoA-degrading enzymes that may have the capacity to offset the CoA biosynthetic pathway may assist in developing novel ways to counteract high and dysregulated CoA levels associated with metabolic diseases. Overall, there is still much to be learned about how and why CoA degradation occurs and its impact on metabolism. 


\section{$\underline{\text { References }}$}

1. Zhang, Y.-M. M., Chohnan, S., Virga, K. G., Stevens, R. D., Ilkayeva, O. R., Wenner, B. R., Bain, J. R., Newgard, C. B., Lee, R. E., Rock, C. O., and Jackowski, S. C. (2007) Chemical knockout of pantothenate kinase reveals the metabolic and genetic program responsible for hepatic coenzyme A homeostasis. Chemistry \& biology 14, 291-302

2. Leonardi, R., Rehg, J. E., Rock, C. O., and Jackowski, S. (2010) Pantothenate Kinase 1 Is Required to Support the Metabolic Transition from the Fed to the Fasted State. PloS one 5

3. Garcia, M., Leonardi, R., Zhang, Y.-M., Rehg, J. E., and Jackowski, S. (2012) Germline Deletion of Pantothenate Kinases 1 and 2 Reveals the Key Roles for CoA in Postnatal Metabolism. PloS one 7

4. Gasmi, L., and McLennan, A. (2001) The mouse Nudt7 gene encodes a peroxisomal nudix hydrolase specific for coenzyme A and its derivatives. The Biochemical journal 357, 33-38

5. Ofman, R., Speijer, D., Leen, R., and Wanders, R. J. C. (2006) Proteomic analysis of mouse kidney peroxisomes: identification of RP2p as a peroxisomal nudix hydrolase with acyl-CoA diphosphatase activity. The Biochemical journal 393, 537-543

6. Reilly, S. J., Tillander, V., Ofman, R., Alexson, S. E., and Hunt, M. C. (2008) The nudix hydrolase 7 is an Acyl-CoA diphosphatase involved in regulating peroxisomal coenzyme A homeostasis. $J$ Biochem 144, 655-663

7. Pierpoint, W. S., and Hughes, D. E. (1954) The synthesis of coenzyme A by Lactobacillus arabinosus 17-5. Biochemical Journal 56, 130-135

8. Tugwood, J., Issemann, I., Anderson, R., Bundell, K., McPheat, W., and Green, S. (1992) The mouse peroxisome proliferator activated receptor recognizes a response element in the 5' flanking sequence of the rat acyl CoA oxidase gene. The EMBO journal 11, 433-439

9. Hasan, S., Platta, H. W., and Erdmann, R. (2013) Import of proteins into the peroxisomal matrix. Front Physiol 4, 261

10. Baker, A., Lanyon-Hogg, T., and Warriner, S. L. (2016) Peroxisome protein import: a complex journey. Biochem Soc Trans 44, 783-789

11. Wang, W., Xia, Z. J., Farre, J. C., and Subramani, S. (2017) TRIM37, a novel E3 ligase for PEX5mediated peroxisomal matrix protein import. J Cell Biol 216, 2843-2858

12. El Magraoui, F., Brinkmeier, R., Mastalski, T., Hupperich, A., Strehl, C., Schwerter, D., Girzalsky, W., Meyer, H. E., Warscheid, B., Erdmann, R., and Platta, H. W. (2019) The deubiquitination of the PTS1-import receptor Pex5p is required for peroxisomal matrix protein import. Biochim Biophys Acta Mol Cell Res 1866, 199-213

13. Platta, H. W., Brinkmeier, R., Reidick, C., Galiani, S., Clausen, M. P., and Eggeling, C. (2016) Regulation of peroxisomal matrix protein import by ubiquitination. Biochim Biophys Acta 1863, 838-849

14. Ley, S. C., and de Carvalho, L. P. S. (2017) Protein CoAlation: a redox-linked post-translational modification. Biochem J 474, 2897-2899

15. Gout, I. (2018) Coenzyme A, protein CoAlation and redox regulation in mammalian cells. Biochem Soc Trans 46, 721-728

16. Chen, W. W., Freinkman, E., Wang, T., Birsoy, K., and Sabatini, D. M. (2016) Absolute Quantification of Matrix Metabolites Reveals the Dynamics of Mitochondrial Metabolism. Cell 166, 1324-1337 e1311

17. Satori, C. P., Kostal, V., and Arriaga, E. A. (2012) Review on recent advances in the analysis of isolated organelles. Anal Chim Acta 753, 8-18

18. Antonenkov, V. D., Sormunen, R. T., and Hiltunen, J. K. (2004) The behavior of peroxisomes in vitro: mammalian peroxisomes are osmotically sensitive particles. American Journal of Physiology - Cell Physiology 287 
19. Merat, S., Casanada, F., Sutphin, M., Palinski, W., and Reaven, P. D. (1999) Western-type diets induce insulin resistance and hyperinsulinemia in LDL receptor-deficient mice but do not increase aortic atherosclerosis compared with normoinsulinemic mice in which similar plasma cholesterol levels are achieved by a fructose-rich diet. Arterioscler Thromb Vasc Biol 19, 1223-1230

20. DeLeve, L. D., Wang, X., Kanel, G. C., Atkinson, R. D., and McCuskey, R. S. (2008) Prevention of hepatic fibrosis in a murine model of metabolic syndrome with nonalcoholic steatohepatitis. Am J Pathol 173, 993-1001

21. Chiang, J. Y. (2013) Bile acid metabolism and signaling. Compr Physiol 3, 1191-1212

22. Desvergne, B., Michalik, L., and Wahli, W. (2006) Transcriptional regulation of metabolism. Physiol Rev 86, 465-514

23. Carreras-Puigvert, J., Zitnik, M., Jemth, A. S., Carter, M., Unterlass, J. E., Hallstrom, B., Loseva, O., Karem, Z., Calderon-Montano, J. M., Lindskog, C., Edqvist, P. H., Matuszewski, D. J., Ait Blal, H., Berntsson, R. P. A., Haggblad, M., Martens, U., Studham, M., Lundgren, B., Wahlby, C., Sonnhammer, E. L. L., Lundberg, E., Stenmark, P., Zupan, B., and Helleday, T. (2017) A comprehensive structural, biochemical and biological profiling of the human NUDIX hydrolase family. Nature communications 8, 1541

24. Calvo, S. E., Clauser, K. R., and Mootha, V. K. (2016) MitoCarta2.0: an updated inventory of mammalian mitochondrial proteins. Nucleic acids research 44, D1251-1257

25. Kerr, E. W., Shumar, S. A., and Leonardi, R. (2019) Nudt8 is a novel CoA diphosphohydrolase that resides in the mitochondria. FEBS Letters 